\title{
Integrated Physics-Based Model for Low Power Bio-Inspired Computing
}

A Dissertation
Presented to
the Faculty of the School of Engineering and Applied Science
University of Virginia
In Partial Fulfillment
of the requirements for the Degree
Doctor of Philosophy (Electrical and Computer Engineering)
by
Dincer Unluer
December 2013




\section{Approval Sheet}

This dissertation

is submitted in partial fulfillment of the requirements

for the degree of

Doctor of Philosophy

\section{Dincer Anluer}

Dincer Unluer

This dissertation has been read and approved by the examining committee:

Avik Ghosh, Adviser

Lloyd R. Harriott, Committee Chair

Benton H. Calhoun, Committee Member

John C. Lach, Committee Member

William B. Levy, Committee Member

Accepted for the School of Engineering and Applied Science:

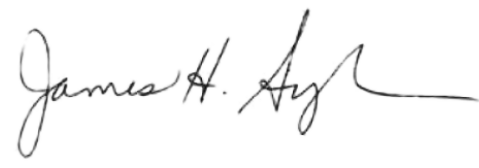

Dean, School of Engineering and Applied Science

December

2013 


\section{Abstract}

In silicon technology, device metrics such as power, delay, and reliability are hitting a brick wall, prompting industry to seek alternative materials, designs, and switching principles. This thesis investigates bio-inspired computing components through a case study that uses signal processing in axonal systems. A physics-based model is developed for each component of the action potential generation in an axon. The two main building blocks of an axon system consist of sequentially activated voltage-gated ion channels (the switch) and adenosine triphosphatase powered ion pumps (the battery). However, the primary aim in this investigation is not to replicate the biological details, but to replace the quantitatively accurate yet empirical operational equations with qualitative, physics-based microscopic models. The primary purposes of focusing on the physics in this study are to deconstruct biological components in terms of solid-state analogues and to understand their potential use for efficient low-power switches, even within conventional Boolean logic. Accordingly, a nano-electro-mechanical field-effect transistor, i.e., a relay, is used to model the ion channel in order to explain how these ion channels are similar to cantilevers that can switch efficiently. The model developed matches the $7.5 \mathrm{mV} /$ decade sub-thermal switching of the low-power sodium channels because it captures both the phase transition from metastable state to stable state and the charge multiplication effect. The electronic ratchets act as the ion pump that uses an energy source (adenosine triphosphate hydrolysis) to power ion flow against a concentration gradient to recharge the battery. This dissertation develops an electronic analogue of a ratchet, essentially a non-equilibrium diode. Furthermore, the research demonstrates that with the ratchet, universal Boolean logic is efficiently implemented and in fact, proves analytically why the electronic ratchet is low power due to ratchet being a voltage-controlled current source. The detailed modeling of the nano-mechanical relays and the ratchets using transport theory identify and conclude the underlying physics behind these devices' power efficient operations and limitations while making connections to the individual components in the axonal networks. 
Anneme ve Babama 


\section{Acknowledgements}

I am deeply grateful to the many people who have helped me complete this dissertation. To all those whom have supported me, including the numerous people whom I am unfortunately unable to mention below, I wish to express my sincerest thanks.

Firstly, I have been incredibly fortunate to work with a truly inspirational advisor, Professor Avik Ghosh. During these formative years, Professor Ghosh has taught me with professional and personal excellence that I strive to embody. From his example, I have learned to become an engineer and a researcher. Only with his guidance and expertise have I been able to complete this work.

Secondly, I wish to recognize all of my VINO Research Group colleagues, especially Frank Tseng, Carlos Polanco, and Kamaram Munira for consistently insightful and inspiring dialogue. I am lucky to have had such a dynamic cohort who made every day of my studies enjoyable.

Thank you to the staff members of the Charles L. Brown Department of Electrical and Computer Engineering at the University of Virginia for their invaluable support through my graduate student experience.

I wish to express my gratitude to my family and friends. Their continued patience and support helped me during every step of this process.

Finally, I would like to dedicate this dissertation to my parents, Zulal Unluer and Erdinc Unluer for their unwavering love, advice, and strength. They have made this thesis, and so many important things in my life, possible. 


\section{Contents}

Contents

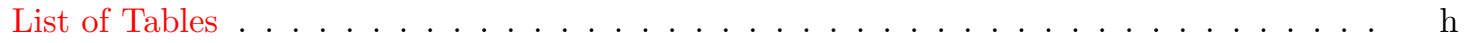

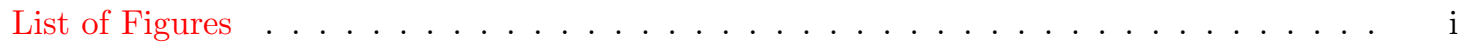

1 Introduction 1

1.1 Beyond CMOS in Solid-state . . . . . . . . . . . . . . . . . 2

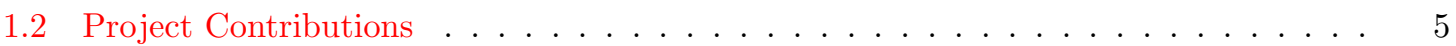

1.3 Biological Devices and Solid-state Analogues . . . . . . . . . . . . . . 8

1.3.1 Non-equilibrium Ratchets as the Ion Pumps . . . . . . . . . . . . . . . . . 9

1.3.2 Nano-electro-mechanical Relays as the Ion Channels . . . . . . . . . . . . . . 11

1.4 Dissertation Overview . . . . . . . . . . . . . . . . . . . . . . . . 12

2 Non-equilibrium Ratchets $\quad 13$

2.1 Ratchet Physics: A Toy Model . . . . . . . . . . . . . . . . . . . . . . . . 14

2.1.1 Asymmetric Potential Creation . . . . . . . . . . . . . . . . . . 16

2.1.2 Approximate Quasi-Analytical Result . . . . . . . . . . . . . . . 18

2.1.3 Simulating a Quantum Ratchet . . . . . . . . . . . . . . . . . . . 19

2.1.4 Simulating a Classical Ratchet . . . . . . . . . . . . . . . . . 20

2.1.5 Calculating the Open-Circuit Voltage . . . . . . . . . . . . . . 23

2.2 Logic with Ratchets . . . . . . . . . . . . . . . . . . . . 25

2.2.1 Static Power in CMOS Versus Ratchet . . . . . . . . . . . . . . . 25

2.2 .2 Dynamic power in CMOS Versus Ratchet . . . . . . . . . . . . . . . . 26

2.2.3 Capacitive Charging Using a Current Source . . . . . . . . . . . . . . . 28

2.2 .4 Adiabatic Charging . . . . . . . . . . . . . . . . . . . . . . . 29

2.2 .5 Ratchet-Based Gates . . . . . . . . . . . . . . . . . . . . . . 32

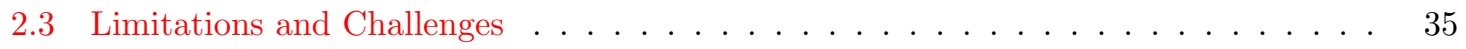

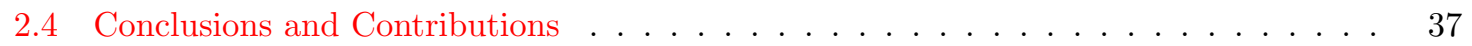

3 Nano-Electro-Mechanical Relays $3 \mathbf{3 8}$

3.1 State of Practice for Nano-Electro-Mechanical FETs . . . . . . . . . . . . . . . 39

3.2 Device Structure . . . . . . . . . . . . . . . . . . . . . . . . . . . . 40

3.3 Transport Equations . . . . . . . . . . . . . . . . . . . . . . 42

3.4 Operation of the Relay . . . . . . . . . . . . . . . . . . . . . . 43

3.5 Sharp Switching and Breaking the Landauer Limit . . . . . . . . . . . . . . . . . 46

3.6 Elasticity versus Dipole and Capacitive Forces . . . . . . . . . . . . . . . . . . . 50

3.7 Hysteresis . . . . . . . . . . . . . . . . . . . . . . . 51

3.8 Connections to the Voltage-Gated Ion Channels . . . . . . . . . . . . . . . . . 54

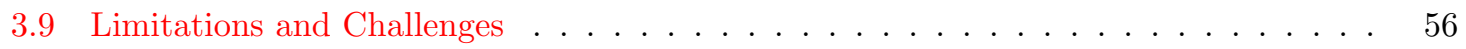

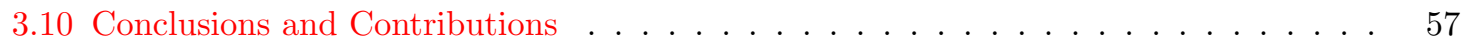


4 Conclusion $\quad 58$

4.1 Summary ............................... . . . . 58

4.2 Future Research Extensions . . . . . . . . . . . . . . . . . . . . . . 59

4.2.1 Bio-Inspired Learning with Nano-Mechanical Relays . . . . . . . . . . . . . . 59

4.2.2 Electronic Ratchet with Peristaltic Asymmetric Potential . . . . . . . . . . . 60

4.2.3 Hysteresis Free Nano-Mechanical Relays . . . . . . . . . . . . . . . . . . . . 61

A Action Potentials: Analog Computing 62

A.1 Information Encoding in the Neurons . . . . . . . . . . . . . . . . . . . 63

A.2 Physics of an Action Potential . . . . . . . . . . . . . . . . . . . . 66

A.3 Hodgkin and Huxley Model . . . . . . . . . . . . . . . . . . . . . . . . . . . 68

A.3.1 Hodgkin and Huxley Circuit and Empirical Equations . . . . . . . . . . . . 70

A.3.2 Fermi Function fitting of Hodgkin and Huxley . . . . . . . . . . . . . . . . 72

$\begin{array}{lr}\text { B Publications } & \mathbf{7 4}\end{array}$

B.1 Journals . . . . . . . . . . . . . . . . . . . . . . . . . . . . . 74

B.2 Book Chapters . . . . . . . . . . . . . . . . . . . . . . . . 74

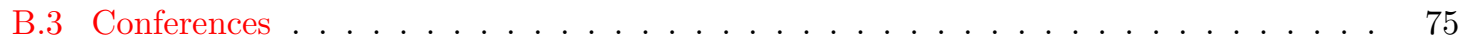

$\begin{array}{lr}\text { Bibliography } & 76\end{array}$ 


\section{List of Tables}

1.1 Carbon device's efficiency compared to Silicon devices in $1 \mathrm{~mm}^{3}$ system $[1] \ldots$

2.1 Simulation Parameters for Drift-Diffusion . . . . . . . . . . . . . . . . 22

2.2 Parameters for Circuit Simulations . . . . . . . . . . . . . . . . . 30

3.1 Simulation Parameters for Model . . . . . . . . . . . . . . . . . . . . . . . . . 44

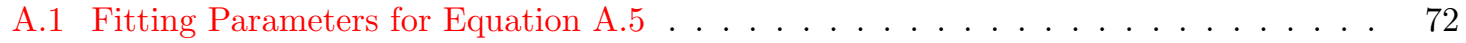




\section{List of Figures}

1.1 The figure above is a typical neuron structure with its components: The dendrite, the nucleus, and the axon. The axon itself consists of ion channels (switches) and ion pumps (batteries) that help the propagation of signals between neurons without any signal decay or signal distortion [2]. The ion channels break the Boltzmann limit of switching seen in solid-state devices [3]. The nano-mechanical relays are similar to the ion channels that open and close pores to allow passive transport of ions from outside of the axon to inside or vice versa in the direction of ionic gradient. Ion pumps work like water molecules moving uphill and are similar to low power mechanical pumps that work against a gradient [4]. These two components help the axon to establish the potential difference between the inside and the outside of the axon in the electrical signal generation. . . . . . . . . . . . . . . . .

1.2 If we zoom into the axon's individual compartments, we can see simple similarities to known mechanisms and components from our daily lives. The ion pumps in the axon are very similar to peristaltic pumps and the ion channels are like the electrically operated relay switches $[5] . \ldots \ldots \ldots \ldots \ldots$

1.3 This figure represents three significant components of axonal networks that generate different parts of the electrical signals. In a typical axon, both the sodium and the potassium channels are closed when the membrane voltage is at resting potential. When external signals in the form of a traveling wave arrive at the axon hillock, they accumulate and start to increase the membrane voltage. When the established predefined threshold voltage is reached, the action potential is generated. As the ion channels close, the ions can no longer flow inward nor outward passively. These ions need to be actively transported in or out of the cell membrane by the ion pumps in order to restore the original balance. . . . . . . . . . . . . . . .

1.4 Alternatives to biological elements in solid-state devices: the sodium/potassium voltage-dependent ion channels $\Longleftrightarrow$ the nano-electro-mechanical relay acting as the passive switch to allow the movement of ions with the gradient (shown in red and green) and the ion pumps $\Longleftrightarrow$ the electronic ratchet performing as an active battery to move ions against the gradient (shown in blue) . . . . . . . . . . . . . .

1.5 Action potential spiking mechanism: The opening and closing phases of the sodium and potassium channels during the initiation of an action potential. First the sodium channels are activated, followed by the activation of the potassium channels, which allows passive transport of ions to create a traveling spike [6]. When the membrane potential undershoots the predefined resting potential, the sodium-potassium ion pumps start to work actively to replenish the signal decay. . . . . . . . . . . . 
1.6 The sodium-potassium ion pump is used in the generation of action potential in the axonal networks by utilizing the non-equilibrium nature of the axon. Ion pumps provide their energy through the hydrolysis of Adenosine-triphosphate molecules and act as an active battery in the axon over a finite noise frequency range. Ion pumps use a saw-tooth shaped flashing potential (shown at right) to move the ions against a concentration gradient [7]. Biological systems are rife with examples of asymmetric potentials that are purported to shuttle elements (typically motor proteins) in the absence of global potentials. This is similar to electronic ratchets in that they transduce local spatial asymmetries into directed currents in the absence of a global bias by rectifying temporal signals that reside far from thermal equilibrium. . . . . . . . .

1.7 A closed view of the potassium channel in the axonal network. Upon activation, the cantilever shaped lid moves conformationally and opens the pores allowing the passive transport of potassium ions. They form energetically potassium-selective pores that span the cell membrane. The diameter of the channel that allows the ions to pass through generates the energetic selectivity of the ions. The ion channels differ with respect to the ions that they allow to pass, such as $\mathrm{Na}^{+}$and $\mathrm{K}^{+}$ion. The notably quick movement of the ions through the pore outperforms the conventional Boltzmann limit in the modern CMOS transistors. . . . . . . . . . . . . . . . . .

2.1 Ion pumps only operate with in the undershoot region of the action potential to replenish the voltage to the original conditions. Because the ion pumps and the electronic ratchets both work against an energy gradient by utilizing the non-equilibrium conditions, a connection can be made. Both devices use a flashing asymmetric potential similar to the uphill movement of a water molecule [4], to move particles or molecules against a gradient. In the ratchets, the asymmetry is created by the interdigitated electrodes, where as in the ion pumps the asymmetry is generated by the sticking and detachment of the ATP molecules. . . . . . . . . . . . . . . .

2.2 Figure above shows the carrier concentration with respect to the effects of the fluctuating asymmetric potential profile. Computing mechanism for a bistate quantum ratchet is simulated with $135 \mathrm{~nm}$ barrier length, $1.67 \mathrm{GHz} \mathrm{AC}$ clock, and $\sim 20 \mathrm{kT}$ barrier height utilizing quantum flow and relaxation. (A) Interdigitated electrodes between two conductor planes creating the desired asymmetric local potential profile in the channel. (B) Initial carrier distribution (shaded area) when potential (dashed line) is "ON". The carriers are localized in the second well and can not diffuse to neither right or left well. (C) Distributed carriers when potential is "OFF". The carriers diffuse in both right and left directions equally. (D) Electron distribution when potential is again turned back on at the heated state. The red line $n^{-}$stands for the carriers overcoming the barrier peak on the left (back-flow), while $n^{+}$stands for those on the right (forward-flow). Since $n^{+}$is bigger than $n^{-}$, a net flow to the right results. (E) Relaxed stage at the end of each cycle, where the electron distribution (n/n0) is reseted to the initial equilibrium solution to cool the system down. . . . . . . . . .

2.3 Prototypical geometry of a ratchet consisting of an interdigitated top gate, which creates the asymmetric time varying potential, and a back gate, which determines the initial number of carriers in the channel by shifting the Fermi level. The current driven by the top gate is used to build up voltage, which can be used to back gate the next cascaded ratchet in the logic architecture. This asymmetric potential profile is very similar to the ion pump potential profile shown in Fig. $1.6 \ldots \ldots \ldots$. . .

2.4 Simulation of the potential profile for a ratchet with $135 \mathrm{~nm}$ barrier length using the Laplace tool box in MATLAB (PDEtool). The positive electrodes are $25 \mathrm{~nm}$ in width whereas the grounded electrodes are $10 \mathrm{~nm}$ in width to increase the asymmetry. The $a / b$ separation factor between the electrodes is 3 . The lower figure shows the Laplace potential through the center of the Si channel. . . . . . . . . . . . . . 
2.5 (A) Spatiotemporal ratchet potential, with a prominent spatial asymmetry with four barriers. (B) Contour-plot of this ratchet potential progressively turned ON between 0.02 and 0.08 ns and then turned OFF after 0.8 ns. (C) Colormap of the current density over this time (blue is negative, red is positive, and green is zero). As seen from the figure, the drift segment of the current during the potential upcycle is actually two phased, showing a quick initial drift down the steeper slope (thin, localized dark blue to cyan), followed by a slower drift of a larger number of particles (hence, a net positive current) down the smaller slope shown in spreading red to yellow. The current density plots shown in (D) are the snapshots at time instances marked by the horizontal dashed lines in (C). During down cycle, the back and forward propagating currents (blue and red, near $t=0.157 \mathrm{~ns}$ ) are comparable as the diffusion process is symmetric in space whereas when potential is turning up, near $t=0.032 \mathrm{~ns}$ back-flow dominates and at $t=0.067 \mathrm{~ns}$ the forward-flow is more prominent. Thus, the net current flow proceeds with a two-phased drift, supplanted gradually by a symmetric

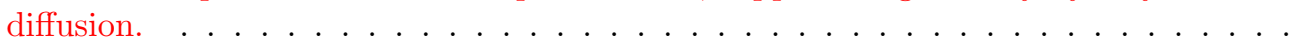

2.6 (A) Asymmetric ratchet potential changes with time and space. (B) Particles are evenly distributed (localized) in bins when the potential is raised and how the particles move to the right with the oscillating top gate (the blue area in between localized bins shows the pathway of particles moving right). (C)-(E) Total, drift, and diffusion current densities. One can see that drift current opposes the diffusion current at all the times, but their magnitudes are different, thus resulting in a net flow to the right. As discussed in the previous sections, the second phase of the two-part drift current shown in yellow moves the particles to right, then the diffusion current spreads the particles evenly right and left. . . . . . . . . . . . . . .

2.7 (A) Drift-diffusion simulation of the electronic ratchet showing the charging of a load capacitor. After 11 clock cycles the particles are distributed equally among all the barriers and there is a net forward-flow of current to the right. The two-phase drift current is less prominent as the $V_{O C}$ is built-up, but still exists as seen in the (B)-(E) (darker red colors start changing to orange/yellow colors as the $V_{O C}$ increases). As the particles reach the right side $(\mathrm{L}=6 \mu \mathrm{m})$, they are ejected by the system and charge a localized back gate capacitor to a $V_{O C}$ of $\approx 145 \mathrm{mV} \ldots \ldots \ldots$. . . . . . . . . .

2.8 Conventional versus adiabatic charging. (A) CMOS circuits are conventionally charged using a DC voltage source. (B) By using a low frequency AC voltage source, the capacitor can be charged while dissipating less energy $[8] \ldots \ldots \ldots \ldots$

2.9 Structure of a single ratchet and its circuit representation, showing the input and output ports of the ratchet modeled a voltage-controlled current source. . . . . . . .

2.10 Equivalent circuit of the charging behavior of the ratchet. The ratchet acts as a voltage-controlled current source, where current is a function of the voltage on the capacitor. The current is at a maximum $I_{m}$ initially when the capacitor is uncharged. As the voltage builds up, the reverse current decreases the net current, until the capacitor reaches the open-circuit voltage, $V_{O C}$ when there is a zero net current. . .

2.11 Circuit model for the ratchet. An adiabatic clock is modeled using an inductor $\mathbf{L}$ and the top gate capacitor $C_{1}$. A (virtual) diode is used to capture the unidirectional current (ratchet) which drives charging of the load capacitor $C_{2} \ldots \ldots \ldots \ldots$

2.12 Energy dissipated as a function of $\omega_{L}$ for a fixed input energy on the inductor. The energy dissipated is calculated by taking the integral of the dissipated power $I^{2} R$ at $t=\infty$. The energy goes to almost zero in the adiabatic limit. . . . . . . .

2.13 Logic circuits are built by cascading ratchet gates together. The first ratchet builds up an open-circuit voltage which is then used to charge or discharge the back gate of the next ratchet. . . . . . . . . . . . . . . . .

2.14 Ratchet inverter computing mechanism. (A, Case 1) $V_{\text {in }}$ is low and moves Fermi level down shown in (B), p-ratchet will have a higher current than n-ratchet so the output will charge the next capacitor $C_{b g}$ until the capacitor voltage reaches $-V_{O C}($ low $)$. $V_{i n}$ is high the output will discharge next capacitor $C_{b g}$ from $V_{O C}$ (high) to zero (A, Case 2). 33 
2.15 (A) Ratchet NAND gate and (B) NOR gate with the extra balance capacitors. Balance capacitors helps the models to have stable transient current, thus eliminating the metastability during the transitions. . . . . . . . . . . . . . . .

2.16 Circuit simulation using the circuit diagram in Fig. 2.11 showing the following results: The voltage being stored on the back gate capacitor compared with the drift-diffusion simulations. For the values of the circuit elements, we use the parameters listed on Table 2.2 and for the Schottky parameters and a barrier height of $15 \mathrm{kT}$ and $I_{0}$ of $1 \mathrm{E}-15 \mathrm{~A}$. Both of the results converge close to $\approx 145 \mathrm{mV}$ and $\approx 160 \mathrm{mV}$, slightly over shooting the expected result of $\approx 125 \mathrm{mV}$. The curves differ in that the circuit model does not capture the back-flow of the electrons as open-circuit builds-up, so it slightly over shoots the actual voltage built-up. . . . . . . . . . . . . . . .

2.17 The ratchet model for creating an asymmetric potential (similar to the electronic ratchet simulations in previous sections) using the ATP hydrolysis to achieve a free Brownian motion [9]. The peristaltic illustration of a protein similar to the ion pumps with two conformational states: One with easy access from the right and one with easy access form the left. The switching between two conformations is induced by the ATP hydrolysis $[9,10] \ldots \ldots \ldots \ldots \ldots \ldots \ldots$

3.1 Ion channels operate in the rising and falling phases of the action potential to steeply increase and decrease the membrane voltage. Because ion channels and nano-electromechanical relays both have the same steep switching characteristics $[3,11]$, a connection can be made. In the ion channels, the cantilever shaped protein lid moves conformationally and opens the pores allowing the passive transport of ions for the expense of one (charge multiplication). In the nano-electro-mechanical FETs, the relay conformationally moves and touches the drain contact to generate a high current jump. 39

3.2 (A) In normally ON device structure, the cantilever starts with contacting both the drain and source electrodes, resulting in active current flow. When a positive gate bias is applied, it moves the cantilever down, increasing the angle "theta". When the cantilever looses it's contact to the drain, the current flow suddenly stops, resulting in the OFF configuration. To turn the device back ON a large reverse bias on the gate is applied until the cantilever makes contact with drain again. (B) Normally OFF device structure where the cantilever starts away from drain. As positive bias on the gate pulls lever down, increasing the angle "theta". Once the cantilever contacts to drain, the current jumps, tuning the device ON. The cantilever is released back to dashed line to the OFF configuration when a large reverse bias is applied on the gate. . . .

3.3 (A) The parabolic potential of the cantilever with one local minimum resulting from the combination of the stiffness and the external field terms. The local minimum shift with the gate bias. (B) The VDW forces resulting from the gate-electrode with a stationary local minimum. (C) A microscopic view of the parabolic potential varying with Gate bias in addition of a localized VDW forces from the contact equals in the NEMFET potential profile, which has one local minimum varying with the gate bias and a second localized minimum (see Table 3.1 for simulation parameters). . . . . .

3.4 (A) Pull-in and pull-out mechanism rising from the NEMFET potential. The pull-in occurs when the positive gate-bias lowers the local maximum (barrier) between the two local minimums allowing the particles to move right (shown by the red curves). (B) The negative voltage shifts the local minimum from the VDW forces to higher energies, thus allowing the particles to shift left to the local minimum from the parabola. 46

3.5 The movement of the minimum, $\theta^{*}$, is shown in the potential profile. The $V_{G}$ shifts the metastable local minimum of the first well, until the local minimum disappears and jumps into the global minimum of the van der Walls well. . . . . . . . . . 
3.6 The transcendental equation obtained by taking $d U\left(\theta^{*}\right) / d \theta^{*}=0$ is shown as the intersection of set of two curves. First set of curves (dashed red) are shifting vertically up as the $V_{G}$ value is increased. For the low values of the $V_{G}$, there are three intersection points corresponding to the three extrema: the local minimum from cantilever, the global minimum from van der Waals, and the local maximum between two wells. At a the high enough $V_{G}$, local minimum and the local maximum collapses resulting with only the global minimum. This movement of the minimums is the main reason of the phase transition in the relays. . . . . . . . . . . . . . .

3.7 Comparison of the subthreshold swing with and without the addition of VDW forces. With the VDW forces the cantilever is pulled down instantaneously shown in red, causing an improved SS of $12 \mathrm{mV} / \mathrm{dev}$. Without the addition of VDW forces from the gate, the fastest the voltage swing is limited to thermal limit of $60 \mathrm{mV} / \mathrm{dev}$ as shown in dashed black line. Thus, proving the importance of the VDW forces at the nano-scale limit on the subthreshold swing. . . . . . . . . . . . . .

3.8 Comparison of the model with the addition and removal of the dipole moments.. With the additional of dipole moments to the model the switching characteristics change. Without the dipole moment the pull-in happens at the $2 / 3$ of the gap, where as with the strong dipole moments, the transition becomes much more linear instead of parabolic and the sticking occurs at $1 / 3$ of the gap. The model captures the exactly the same switching behavior as in [12] when the dipole moment is not included and the same design parameters are used (shown as dashed.) . . . . . . . . . . . .

3.9 (C) The numerical solution of the Fokker-Plank equation coupled with the Landauer's theory results in switching voltages that are dependent on the scan rate. As the scan rate $\left(1 / T_{\max }\right)$ increases, the switching voltages shifts to right, more positive values, causing a hysteresis. (B) When the scan rate is fast enough, the particles do not have time to follow the potential, resulting in a delayed version of the Boltzmann distribution. $T_{\max }=1$ represents the case with no hysteresis where the scan rate and the movement speed of the particles are comparable (Particles can follow the rate of change in the potential) (A). $T_{\max }=0.25$ has a faster scan rate than the particles can follow resulting in a delay in transition causing it to switch at higher gate bias. . . .

3.10 When the switching probabilities of the ion channels are investigated in membrane voltage versus probability rather than the conventional time versus probability, there is a distinguished hysteresis loop in all the switching probabilities ("n", "m", and "h"). These probability loops also result in a hysteresis loop in the sodium and potassium currents. These loops keep repeating themselves every time the axon compartment fires a new action potential. . . . . . . . . . . . . . . .

3.11 Comparison of the action potential hysteresis versus NEMFET hysteresis. Because of the overshoot of the potassium gates in the axon (dead-time), the loops differ in shape. The hysteresis is seen in both systems when plotted in current versus voltage. In the NEMFETs, the hysteresis occurs due to the different attaching and detaching voltages (due to the van der Walls forces). In the action potentials, it occurs due to the individual hysteresis loops in the sodium and potassium currents. NEMFET currents would be a better comparison with a single ion channel (sodium or potassium), since the turning $\mathrm{ON}$ and $\mathrm{OFF}$ voltages differ similar to the nano-mechanical relays. . . .

3.12 Matching of the steep switching in the voltage-controlled sodium ionic channels [3] and the nano-mechanical relays. With the use of dipole forces changing the shape of the proteins allowing passive transport through, the sodium channels can switch with almost $7.5 \mathrm{mV} /$ decade. If we use the nano scale dimensions where the dipole forces are much more prominent than the electrostatic forces, we achieve comparable steep switching of $6.5 \mathrm{mV} /$ decade in nano-mechanical relays. This proves that the NEMFETs can be used to model the ion channels and demonstrates their steep and low power switching behaviors. . . . . . . . . . . . . . . 
4.1 Control flow of the synapses composed of a three terminal nano-mechanical relay. The naturally occurring hysteresis in a current-voltage relationship can be utilized to implement the STDP learning mechanism. The all-or-nothing change in the conductance of the channel by applying a gate voltage can aid the system in memorizing conductance and can be maintained without fluctuations. Pre-voltage and post-voltage signals can be controlled by using the circuitry shown in [13] . . . . . . . . . . . .

4.2 This figure shows the peristaltic movement wave of the esophagus during swallowing, which allows the food to move in one direction. A possible implementation of peristalsis on the electronic ratchets is using multi-phase clocks (The turning ON of the first clock is followed by the turning ON of a phase-shifted second clock as the first clock turns OFF). This multi-clock system allows a net flow to the right with minimal back-flow of particles. Since the particles always drift from one well to the next and do not have time to diffuse equally, the ratchets in smaller scales can be implemented without the need of $\mathrm{THz}$ clock frequencies. . . . . . . . . . . . . . . . . .

4.3 With the addition of the third contacts, the effect of the hysteresis begins to disappear as the voltage-bias is increased. Once the depth of the well is the same as the asymmetric potential of the third ratchet-like interdigitated contacts, the pull-in and pull-out are collapsed, thus enabling the use of nano-mechanical-relays for digital applications. As seen in the simulations above, the asymmetric potential removes the van der Walls well and enables the particles to drift back to the original potential well.

A.1 The voltage-controlled potassium (purple) and sodium (orange) ion channels. They mechanically change shape when the sodium $\left(\mathrm{Na}^{+}\right)$rich positive potential on the outside from the potassium $\left(K^{+}\right)$rich potential on the inside of a axon is changed by an external pulse. They allow transport of the ions passively where as the adenosine triphosphatase based sodium-potassium ion pump within a cell membrane restores the membrane potential actively. . . . . . . . . . . . . . .

A.2 The information (bits) in neural networks is stored between the time intervals of two successive electrical pulses. Neurons use this different differential pulse position modulation technique since it is the most power efficient way of encoding information. Two main ways to maximize the bit-rate per joule in the neural networks: First by squeezing the pulse width in the cost of increasing the overall cost of spike generation. Second by decreasing the time between two successive pulses in the cost of distorting the information stored between two pulses. . . . . . . . . . . . .

A.3 The information (bits) in neural networks is stored between the time intervals of two successive electrical pulses. Neurons use this different differential pulse position modulation technique since it is the most power efficient way of encoding information. Two main ways to maximize the bit-rate per joule in the neural networks: First by squeezing the pulse width in the cost of increasing the overall cost of spike generation. Second by decreasing the time between two successive pulses in the cost of distorting the information stored between two pulses. . . . . . . . . . . . . .

A.4 Bits per second vs. ISI: As the time between two successive pulses increase, the number of Bits/Sec decreases assuming the gamma distribution with varying the mean. Shorter the separation between the more bits per second the axonal network can carry. Energy vs. ISI: As the time between two successive pulses increase, the energy of generating a spike changes also since the overlap of sodium and potassium currents differ. There is a peak point in which the cost of generating a spike becomes too expensive, so the neurons optimized by the axon properties avoid generating spikes at tho given ISI. . . . . . . . . . . . . . . . . . . .

A.5 As the time between two successive pulses increase, the velocity of the action potential generated changes due to the distortion between the two signals. This change in the velocity has a huge impact on the energy of generating a spike changes since the overlap of sodium and potassium currents differ. As seen in the figure, for a slower spike, the overlap increases thus the overall cost of generating single spike goes up. . 
A.6 Action potential spiking mechanism: Opening and closing times of the sodium and potassium channels during the initiation of an action potential. First the sodium channels are activated and followed by the potassium channels to create a traveling

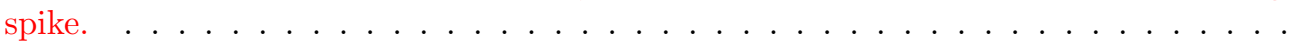

A.7 Ultrafast potassium channel activation of $7.5 \mathrm{mV} /$ decade from the Hodgkin and Huxley experiments. The channel goes under a conformational change to create a physical gap for the ions to pass through the channel membrane. . . . . . . . . . . . .

A.8 The Hodgkin and Huxley electrical equivalent circuit for a squid giant axon. The capacitor represents the capacitance of the cell membrane; the two variable resistors represent voltage-dependent $\mathrm{Na}^{+}$and $\mathrm{K}^{+}$conductances, the fixed resistor represents a voltage-independent leakage conductance and the three batteries represent reversal potentials. The probability of opening and closing of the ion gates are similar to the FETs. Sodium gate is regulated by $m(t)$ like a NMOS and $h(t)$ like a PMOS. $m(t)$ to third exponent represents the fast opening of the sodium gate where as $\mathrm{h}(\mathrm{t})$ represents slow shutting of the sodium gate. . . . . . . . . . . . . . .

A.9 The numerical solution of Eq. A.1 of the Hodgkin and Huxley model showing the membrane voltage vs. time after the axon is excited by an external pulse. The $m(t)$, $n(t)$, and $h(t)$ show the probability of an ion channel opening and closing in time and Eq. A.2 and Eq. A.3 are used to calculate the values. The $m$ represents the probability of the fast-time scaled sodium channels opening where as the $n$ shows the potassium channel probability. $h$ demonstrate the slow time-scale closing of the sodium channels. 71

A.10 The fitting of the rate constants with the Fermi-Dirac equations, resulting in a almost perfect fit of the current-voltage curves of a firing action potential. The individual currents for sodium, potassium, and leak and the gating particles $m(t), n(t)$, and $h(t)$ are with in one percent fitting tolerance when the Eq. A.5 is used instead of Eq. A.3. 


\section{Chapter 1}

\section{Introduction}

The International Technology Roadmap for Semiconductors predicts that intrinsic device properties such as leakage, power, and delay of complementary metal-oxide-semiconductor (CMOS) technology are at the point of reaching their physical limits. This brick wall is compelling industry to invest in and explore novel materials to replace or complement silicon [14]. As the aspect dimensions get smaller, the two major hindrances in state-of-the-art CMOS technology are the Boltzmann-ShannonLandauer limit of $60 \mathrm{mV} /$ decade subthreshold swing (SS) at room temperature and the increase in both dynamic and static power dissipation. Dynamic power is proportional to $C_{\text {Load }} f V_{D D}^{2}$, where lowering the supply-voltage $\left(V_{D D}\right)$ technically reduces the power consumption. Static power splits into two major parts: gate leakage that can be enhanced by the use of high-k gate dielectric materials and subthreshold leakage that can be improved by improving the channel doping profile. However, new devices that truly lower subthreshold and gate leakage while operating at lower supply voltages are required to solve the power dissipation problem completely.

An ideal solution would allow almost zero leakage in OFF stage while expending less power during ON stage and while processing with minimal delay without degrading the performance and reliability of the system. An important example of this ideal solution can be biological networks, such as the nervous system, that utilize many different devices and concepts to overcome the energy trade-off problem (See Fig. 1.1). The nervous system utilizes steep-switching devices and ambient thermal energy to lower power consumption. Axonal networks also use various devices (such as simple, everyday pumps and relays as seen in Fig. 1.2) and coding mechanisms to improve their efficiency, speed, and power consumption. Because the information carriers are massive ions rather than 


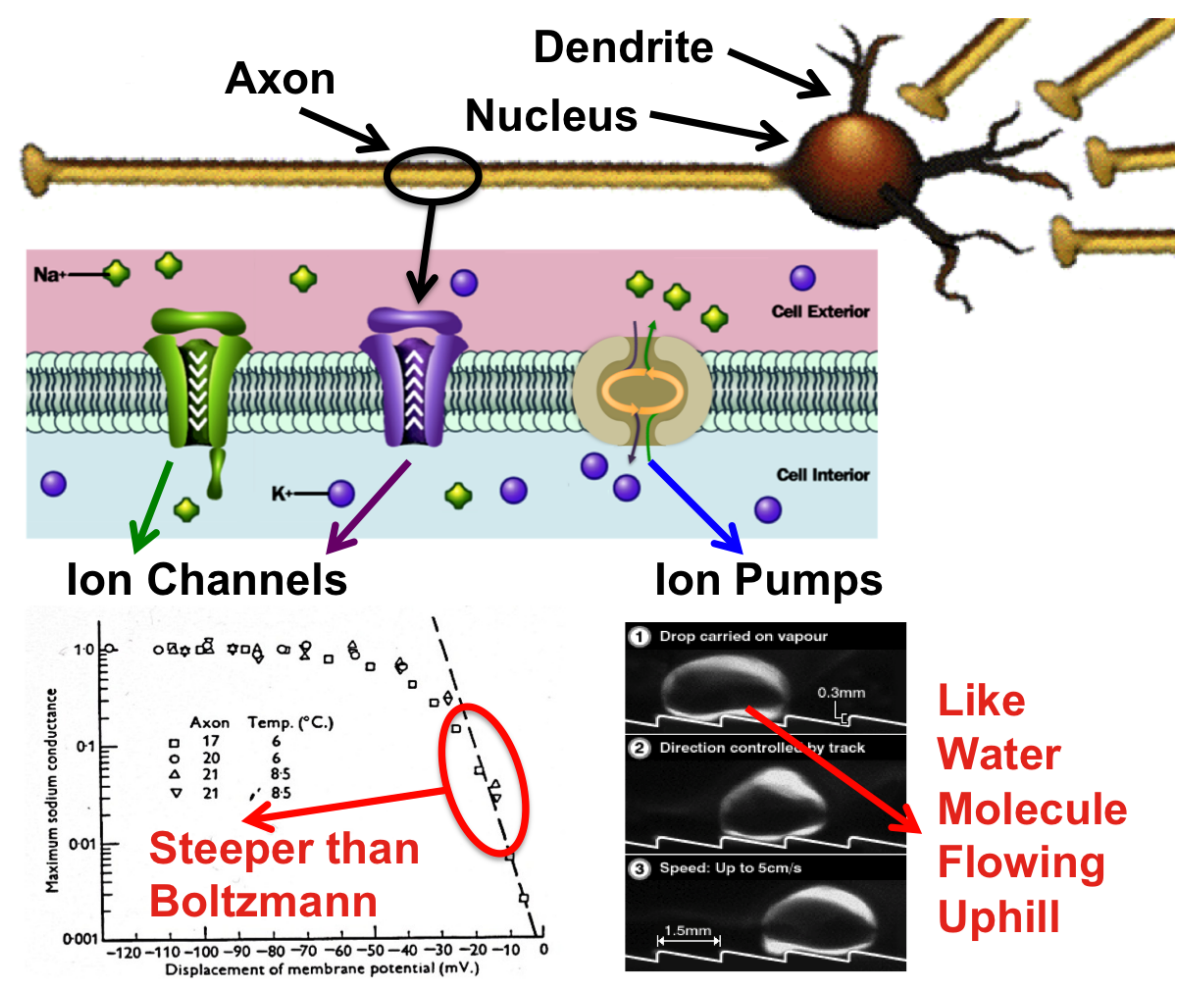

Figure 1.1: The figure above is a typical neuron structure with its components: The dendrite, the nucleus, and the axon. The axon itself consists of ion channels (switches) and ion pumps (batteries) that help the propagation of signals between neurons without any signal decay or signal distortion [2]. The ion channels break the Boltzmann limit of switching seen in solid-state devices [3]. The nanomechanical relays are similar to the ion channels that open and close pores to allow passive transport of ions from outside of the axon to inside or vice versa in the direction of ionic gradient. Ion pumps work like water molecules moving uphill and are similar to low power mechanical pumps that work against a gradient [4]. These two components help the axon to establish the potential difference between the inside and the outside of the axon in the electrical signal generation.

electrons, the key methods to increasing speed in biological networks are the multi-wire representation of information and parallel information processing. State-of-the-art CMOS technology can only achieve lower subthreshold, gate leakage, and supply voltages at the expense of device performance, causing industry to shift toward multi-core processors similar to biological systems (specially the brain) in order to recover system throughput [15].

\subsection{Beyond CMOS in Solid-state}

In more recent decades, development in semiconductor industry has progressed with the transistor density guidelines of Moore's Law. Many engineers and scientists are working toward new designs, new switching paradigms, and turning to innovative materials to replace the conventional CMOS. Non-charge-based devices are investigated as a possible alternative. For example, spin-based logic 


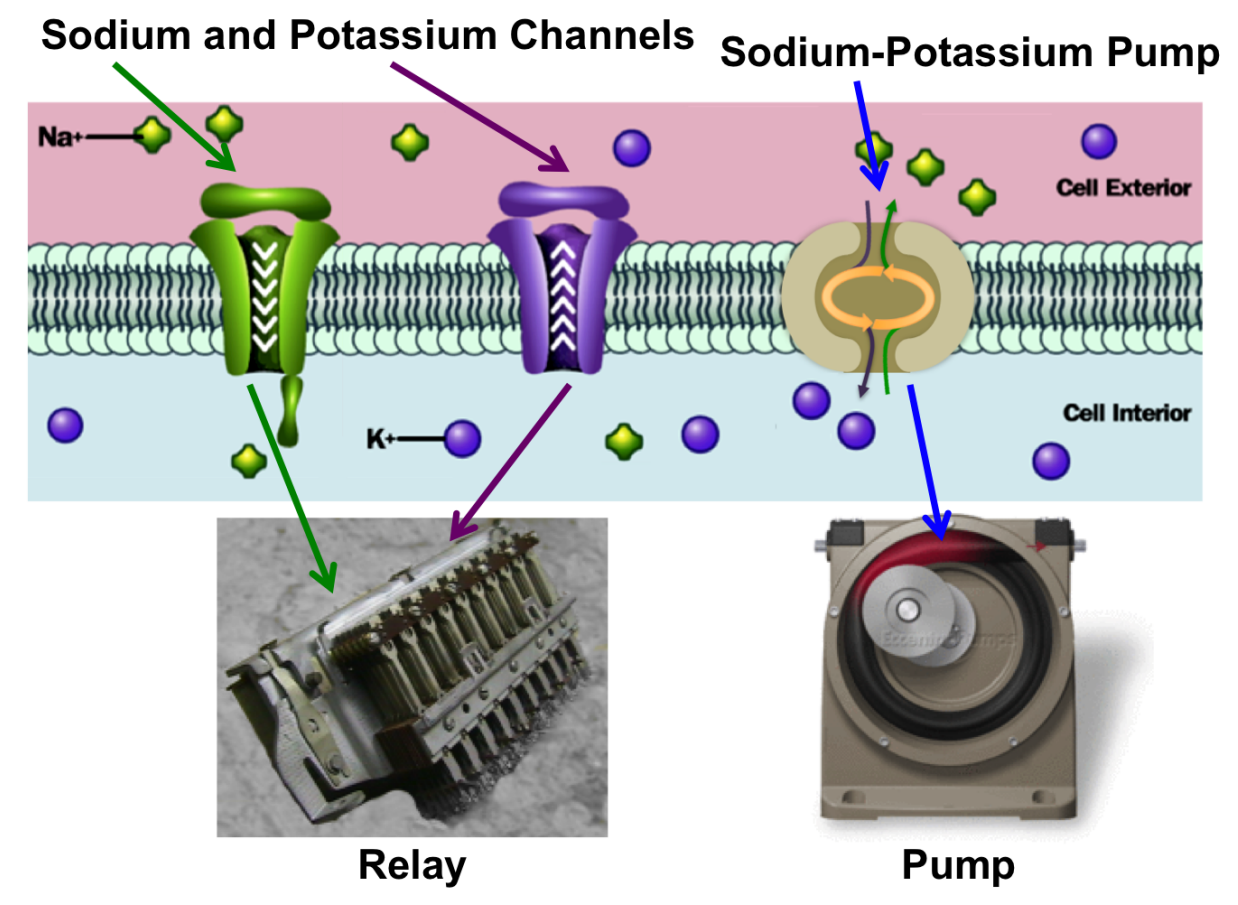

Figure 1.2: If we zoom into the axon's individual compartments, we can see simple similarities to known mechanisms and components from our daily lives. The ion pumps in the axon are very similar to peristaltic pumps and the ion channels are like the electrically operated relay switches [5].

stores information by using the electron's spin degree of freedom in a free layer of a Magnetic Tunnel Junction. The spin transfer, as opposed to the charge transfer, propagates the signal. As a result, these spin devices use less power and are non-volatile. The directions of the two spins of the ferromagnetic films can be switched individually by applying an external magnetic field, allowing fast write and read [16, 17]. However, its limitations are the switching field strengths to avoid spontaneous switching and maintaing low write-error-rates. Up to the present time, the error-rates in spin-based logic are higher than the required reliability standards of CMOS industry [18].

A number of non-silicon channel materials are being considered as a replacement for silicon-channel devices. These non-silicon options include typical semiconductor materials such as $\mathrm{SiGe}$ or Ge, and various III-V material combinations such as GaAs. Advantages of these semiconductor materials include lower scattering under stress and lower effective mass, which permits higher bulk electron and hole mobility. However, these semiconductor materials lack good electrostatic control due to fabrication challenges of depositing high quality gate dielectrics on the channel [19]. Another major challenge is developing a low-cost and defect-free process technology to deposit III-V materials and Ge on top of the silicon wafers. 
Carbon-based electronics, mainly carbon nanotube (CNT) and its planar form graphene, are possible candidates. CNTs used as interconnects exhibit excellent intrinsic performance, high carrier mobility, and high current density, while CNT field-effect-transistors (FETs) exhibit high gain and can be considered novel devices [20, 21]. Various CNT-FETs have been studied, but due to their large intrinsic contact resistance, lack of chirality control, and difficulty in patterning and aligning multiple tubes to build circuits, CNT-FETs have not been a viable replacement for silicon in mass production [22, 23]. Given the semiconductor industry's significant investment in planar fabrication techniques, solutions compatible with current industry practice are clearly preferable.

Due to its planar-fabrication techniques and properties similar to CNTs, intrinsic graphene emerges as a serious contender for the post-silicon era. However, the biggest setback of using intrinsic graphene in digital applications is the absence of a bandgap, which is required to distinguish between high and low current states for reliability in digital logic [24]. Various experiments have demonstrated the opening of a bandgap by either applying a vertical electrical field on bilayer graphene [25, 26], or by using quantum confinement in the form of narrow graphene nano-ribbons ( $<10 \mathrm{~nm}$ in width) [27, 28]. However, bandgap opening comes at the expense of graphene mobility [29]. Additionally with current fabrication techniques, the typical fabricated graphene transistors have bandgaps that are less than $0.4 \mathrm{eV}$, lack of saturation, and the presence of tunneling currents that prevent complete OFF states for both n-FET and p-FET [27, 30].

Previous research has also focused on finding alternatives to conventional CMOS Boolean logic by using electronic ratchets [8]. Electronic ratchets are devices that are not bound by equilibrium conditions. Ratchets extend the principles of Brownian motors in order to charge carrier systems. Brownian motors, when placed under non-equilibrium conditions, can harness thermal fluctuations for directed motion $[31,32]$. Electronic ratchets are energy efficient voltage dependent current sources, similar to low power ion pumps in an axon, that require minimal energy to restore the system back to equilibrium conditions in the axon. Ratchets are very amenable to being used in alternative logic design, because they operate at slower speeds than the industry's clock requirements for high performance computation. For example the binary decision diagram (BDD), which is used to guide the majority of electron flow directionally to outputs and the majority gate logic, returns a true value if more than $50 \%$ of the inputs are true [33]. 
Alternative transistor designs such as tunnel FETs are being investigated as a possible replacement for conventional silicon based transistors [34]. Tunnel FETs use band-to-band tunneling, an electron in the valance band tunnels across the band gap to the conduction band without the assistance of traps. The band gap acts as the source-to-drain potential barrier that the particle tunnels across, thus managing experimentally to achieve SS lower the $60 \mathrm{mV} /$ decade [35]. The greatest challenge with tunnel FETs is achieving high performance (high ON current) without degrading the OFF current and maintaining an $\mathrm{SS}$ of less than $60 \mathrm{mV} /$ decade [36]. This non-zero OFF current results in a lower limit in energy efficiency due to high static power consumption.

An additional example of low subthreshold devices is Nano-electro-mechanical FETs (NEMFETs). NEMFETs are conformational transistors, meaning that the gate controls the current through the angular movement of the conducting cantilever [37]. In CMOS, the gate controls the induced charge. The charges are thermionically propagated from the source to the drain over a barrier, thus limiting the steepest transition to $60 \mathrm{mV} /$ decade. However, in the NEMFETs, the applied gate voltage controls the movement of the lever instead of the channel resistance. The conformational movement of the nano-cantilevers results in zero gate leakage current, zero OFF current, and most importantly, $\mathrm{SS}<<60 \mathrm{mV} /$ decade switching due to phase transition from metastable state to stable state and charge multiplication [38]. NEMFETs have been used to implement Boolean logic with almost zero-leakage current, solving one of the major problems of the industry [39]. These cantilevers confront the challenge of achieving aggressive dimensions without adhesion issues. Previous studies have indicated that nano-mechanical relays can be integrated with the CMOS for improved stability and low leakage [40].

\subsection{Project Contributions}

In order to further analyze and understand how biological devices are more power efficient than silicon devices, we replace the quantitatively accurate yet empirical biological equations with the

Table 1.1: Carbon device's efficiency compared to Silicon devices in $1 \mathrm{~mm}^{3}$ system [1]

\begin{tabular}{|c|c|c|}
\hline Memory & $3 \times 10^{3} \mathrm{bit}\left(10^{16} \mathrm{bit} / \mathrm{cm}^{3}\right)$ & $\sim 10^{7} \mathrm{bit}\left(10^{19} \mathrm{bit} / \mathrm{cm}^{3}\right)$ \\
Logic & $3 \times 10^{4} \mathrm{bit}\left(10^{17} \mathrm{bit} / \mathrm{cm}^{3}\right)$ & $\sim 10^{6} \mathrm{bit}\left(10^{18} \mathrm{bit} / \mathrm{cm}^{3}\right)$ \\
Energy per bit & $10^{-18} \mathrm{~J}$ & $10^{-20} \mathrm{~J}$ \\
Power & $10^{-9} \mathrm{~W}$ & $10^{-13} \mathrm{~W}$ \\
\hline
\end{tabular}




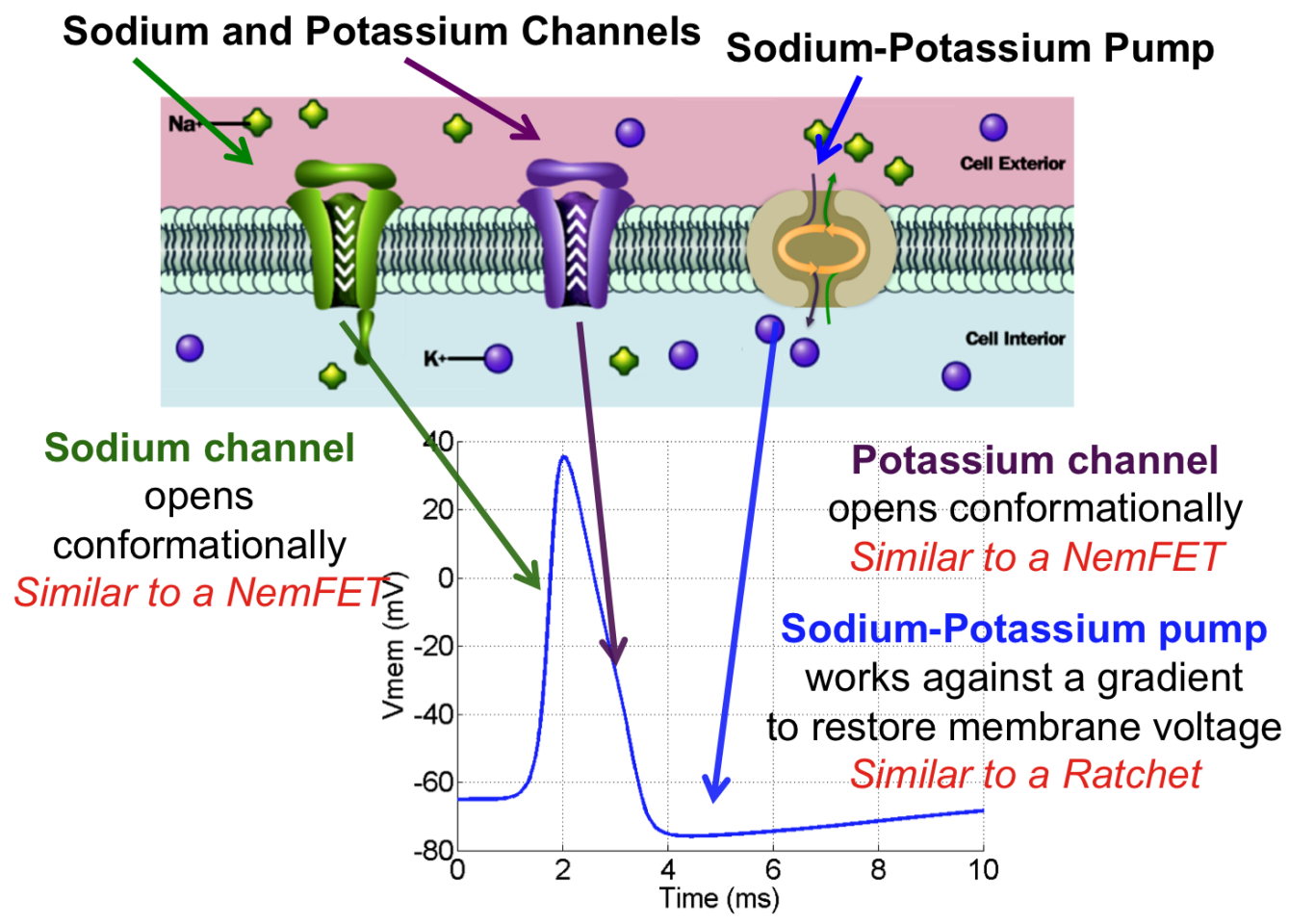

Figure 1.3: This figure represents three significant components of axonal networks that generate different parts of the electrical signals. In a typical axon, both the sodium and the potassium channels are closed when the membrane voltage is at resting potential. When external signals in the form of a traveling wave arrive at the axon hillock, they accumulate and start to increase the membrane voltage. When the established predefined threshold voltage is reached, the action potential is generated. As the ion channels close, the ions can no longer flow inward nor outward passively. These ions need to be actively transported in or out of the cell membrane by the ion pumps in order to restore the original balance.

qualitatively, physics-based microscopic models. The complexity of the nervous systems makes it necessary to look at different levels of abstractions. Table 1.1 illustrates how biological devices have superior memory, logic, energy per bit, and power properties when compared to silicon devices in a 1 $\mathrm{mm}^{3}$ system. The individual axonal network elements work together to create non-decaying electrical signals called the action potential (AP). As seen in Fig. 1.3, generation of an AP is divided into two significant components: the voltage-dependent ion channels that work passively with the voltage gradient (switch) and the ion pumps that work actively against the gradient (battery) [41]. By modeling these low power axonal devices' solid-state counterparts, we derive connections, limitations, and propose alternative devices for the future of modern electronics. We demonstrate the physics behind the operation of individual elements in the axonal networks and prove the reasons as to why they operate efficiently at low power. 


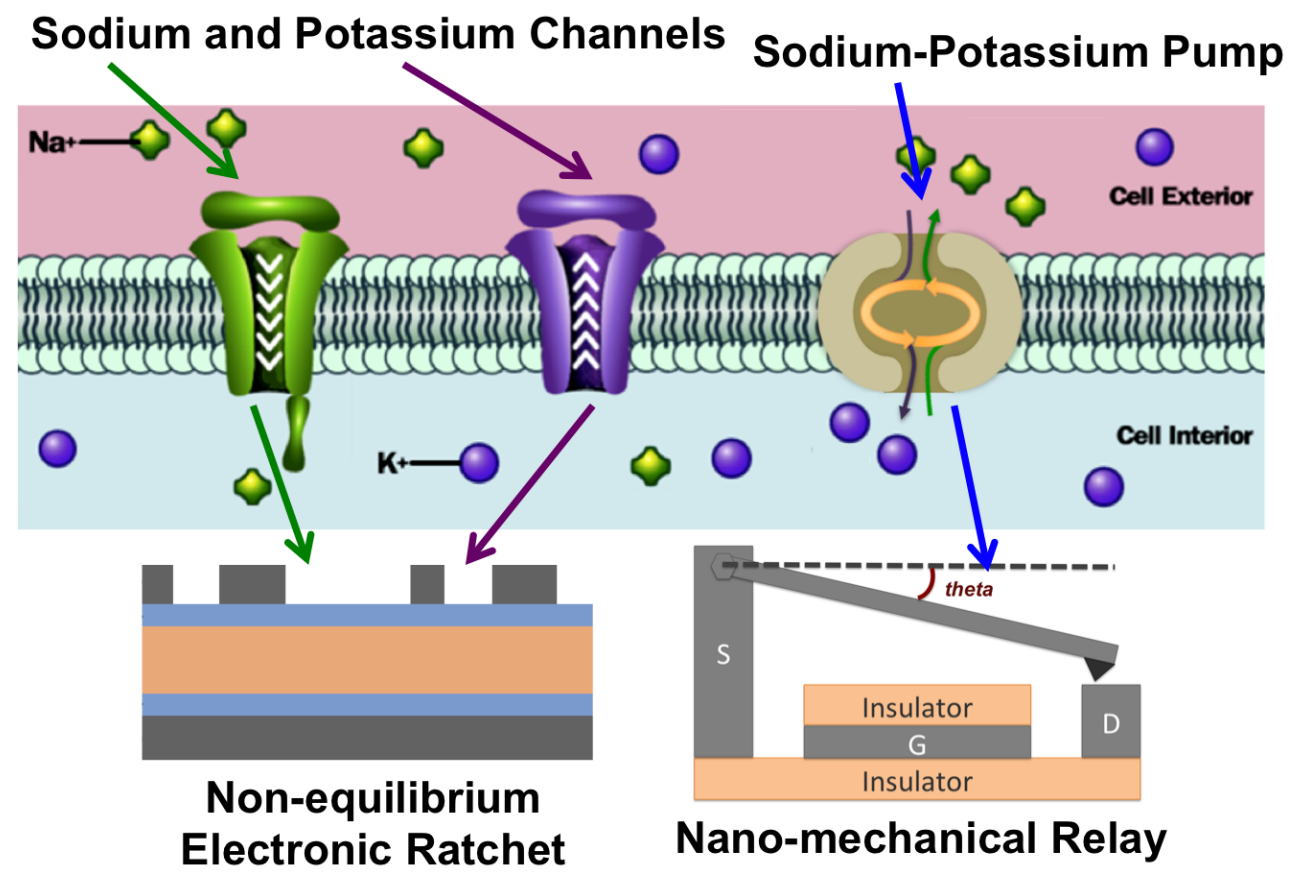

Figure 1.4: Alternatives to biological elements in solid-state devices: the sodium/potassium voltagedependent ion channels $\Longleftrightarrow$ the nano-electro-mechanical relay acting as the passive switch to allow the movement of ions with the gradient (shown in red and green) and the ion pumps $\Longleftrightarrow$ the electronic ratchet performing as an active battery to move ions against the gradient (shown in blue).

To aid in the understanding of the biological system's power efficiency, we model similar solid-state analogues that focus on physics in order to deconstruct biological components. We demonstrate that the solid-state analogues of the ion pumps are electronic ratchets and that the ion channels are the nano-mechanical relays (as seen in Fig. 1.4). In this work, the non-equilibrium electronic ratchet is modeled within both the classical and quantum limits. Ratchets with an asymmetric potential profile are calculated to move particles without the use of drain bias, thus lowering static dissipation. Additionally, since the ratchets function as a gate voltage-controlled current source, they also have the potential to reduce the dynamic dissipation that is associated with charging and discharging capacitors [42]. However, the speed and clock frequency versus size scaling can limit the overall performance of the electronic ratchet in modern electronics.

The ion channels' replacement, dipole-coupled nano-electro-mechanical FETs, are modeled by using the Landauer based transport model for electrons. The dynamics are driven by a thermal transition between a metastable and a stable ground state in the energy landscape. This landscape is composed of the elastic energy of the cantilever and the dipolar torque exerted by a van der Waals interaction at the drain. The individual pull-in and pull-out phases demonstrate a remarkably low 
subthreshold swing driven by both a dipole-based charge multiplication effect, and an abrupt phase transition that closes the air gap between the end of the cantilever and the drain [38] The hysteresis loop, mechanical delay, and loss of elasticity as cantilever scale down in size limit the performance of nano-mechanical relays in the semiconductor industry.

\subsection{Biological Devices and Solid-state Analogues}

Understanding the solid-state's connections to axonal devices requires delving into the individual current components that comprise action potentials. When not firing an AP, the lipid bilayers in the axon separate a sodium rich positive potential on the outside from a potassium rich negative potential in the inside. This initial potential difference across the membrane is called the "resting potential", meaning both the sodium and the potassium channels are shut and the ion pumps are inactive (seen in Fig.1.5A). The "depolarization" phase starts, when an external signal in the form of a traveling wave arrives and increases the membrane potential by a predefined amount. The

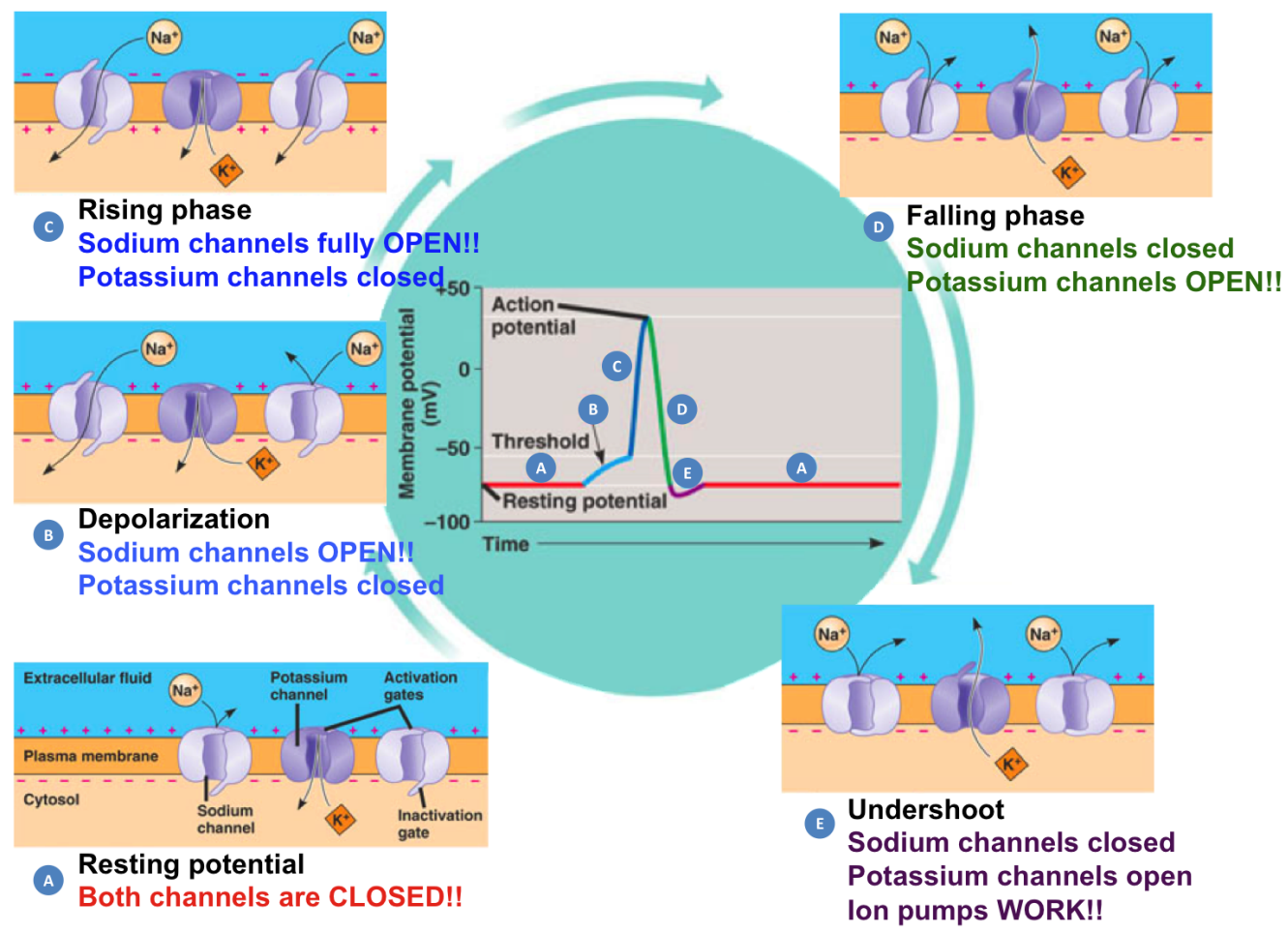

Figure 1.5: Action potential spiking mechanism: The opening and closing phases of the sodium and potassium channels during the initiation of an action potential. First the sodium channels are activated, followed by the activation of the potassium channels, which allows passive transport of ions to create a traveling spike [6]. When the membrane potential undershoots the predefined resting potential, the sodium-potassium ion pumps start to work actively to replenish the signal decay. 
depolarization phase generates an electromagnetic field strong enough to distort the shape of the channel protein (the relay) so that it opens up a physical cavity. Through this gap the $\mathrm{Na}^{+}$ion's influx begins across the membrane, as shown in Fig.1.5B. The sodium ions move from a high concentration to a low concentration to create equilibrium. The continued inflow of sodium ions, further increases the membrane potential and opens more sodium channels. Opening of all available sodium channels causes a rapid upswing of membrane potential known as the "rising" phase (seen in Fig.1.5C).

Meanwhile, the passive influx of sodium ions reverses the polarity of the membrane, causing the sodium channels to deactivate and the potassium channels to activate. Potassium channels open by mechanically moving the protein relay, triggering a passive outward flow of the $K^{+}$ions. Consequently, the absolute value of membrane potential decreases. This is called the "falling" phase, as shown in Fig.1.5D. The electrochemical potential returns to resting value and overcompensates the membrane potential. This overcompensation is due to the fact that the potassium channels do not respond immediately to the voltage change, and therefore close more slowly. This transient negative shift in membrane potential is due to additional potassium currents and is called the "undershoot" or "dead-time" (shown in Fig.1.5E). The net outcome is a voltage pulse with a characteristic shape that propagates directionally along the axon, alternatively opening and closing the switches.

Ultimately all the channels (switches) close and a series of sodium-potassium pumps (battery) start working to move ions. Ion pumps replenish the signal voltage decay by carrying ions actively against a voltage gradient through the hydrolysis of Adenosine-triphosphate (ATP) molecules. As the ion pumps move three sodium ions from the inside of the cell to the outside of the cell, two potassium ions are simultaneously transferred from the outside of the cell to inside of the cell. This unbalanced charge transfer contributes to the charge separation across the membrane and increases the membrane voltage back to the resting potential as demostrated in Fig.1.5A.

\subsubsection{Non-equilibrium Ratchets as the Ion Pumps}

In this work, electronic ratchets are used to replicate and model the properties of ion pumps that operate at low energies. Importantly, in ion pumps both sodium and potassium ions travel from areas of low concentration to areas of high concentration (each ion is moving against its concentration gradient). Ion pumps harvest energy from the applied field by means of field-induced conformational fluctuations as demonstrated by Brownian ratchets. Ion pumps utilize the non-equilibrium nature of 

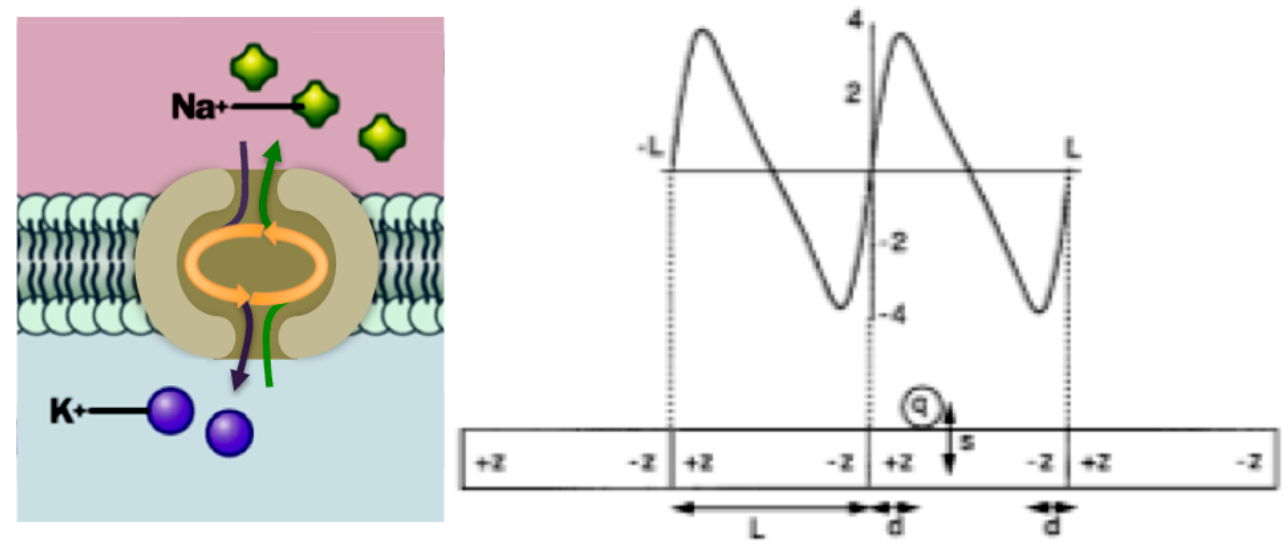

Figure 1.6: The sodium-potassium ion pump is used in the generation of action potential in the axonal networks by utilizing the non-equilibrium nature of the axon. Ion pumps provide their energy through the hydrolysis of Adenosine-triphosphate molecules and act as an active battery in the axon over a finite noise frequency range. Ion pumps use a saw-tooth shaped flashing potential (shown at right) to move the ions against a concentration gradient [7]. Biological systems are rife with examples of asymmetric potentials that are purported to shuttle elements (typically motor proteins) in the absence of global potentials. This is similar to electronic ratchets in that they transduce local spatial asymmetries into directed currents in the absence of a global bias by rectifying temporal signals that reside far from thermal equilibrium.

the axon and the correlated $\mathrm{AC}$ field across the membrane to generate an active ionic current. The sticking and unsticking of the ATP molecules produce a potential profile in the pump that is similar in appearance to a saw-tooth profile (Fig. 1.6) [7]. Although the hydrolysis of the ATP is an active process that expends energy, when compared with conventional logic circuits, the power dissipated from the systems is limited (both static and dynamic dissipation) [43].

The electronic ratchet extends the principles of Brownian motors to charge carrier systems. Brownian motors, when placed under non-equilibrium conditions, can harness thermal fluctuations for directed motion $[32,44]$. Brownian motors are found across diverse areas of research that range from ion pumping in artificial nano-pores [45], to biological motor proteins [46]. Likewise, the electronic ratchet uses an asymmetric saw-tooth potential (see Fig. 2.4) to first drive carrier concentrations away from equilibrium and then to lower the potential over time allowing the carriers to diffuse (see Fig. 2.2). When the potential is again raised, the asymmetry of the potential causes more charges to drift in one direction than the other, resulting in a net current flow (see Fig. 2.6). This process is very similar to the movement of ions against the gradient in ion pumps. 

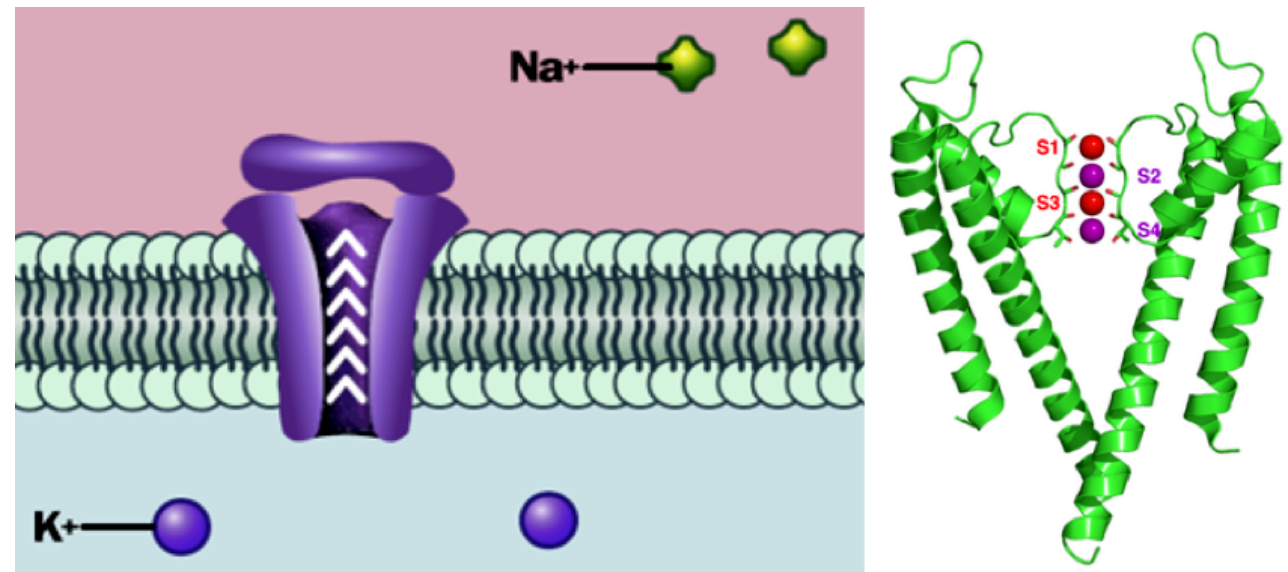

Figure 1.7: A closed view of the potassium channel in the axonal network. Upon activation, the cantilever shaped lid moves conformationally and opens the pores allowing the passive transport of potassium ions. They form energetically potassium-selective pores that span the cell membrane. The diameter of the channel that allows the ions to pass through generates the energetic selectivity of the ions. The ion channels differ with respect to the ions that they allow to pass, such as $\mathrm{Na}^{+}$and $K^{+}$ion. The notably quick movement of the ions through the pore outperforms the conventional Boltzmann limit in the modern CMOS transistors.

\subsubsection{Nano-electro-mechanical Relays as the Ion Channels}

Nano-mechanical relays have similar properties to ion channels. These ion channels are more power efficient than the state-of-the-art CMOS switches due to their small SS and extreme low OFF currents. The ion channels are conformational proteins that help shape action potentials by controlling the flow of ions across the cell membrane. Two very significant features of the ion channels cause them to be important proteins for axonal networks. Firstly, the rate of ion transport through the channel is very high. Secondly, the ions pass through the channels from a high electrochemical gradient to a low electrochemical gradient. This passive passage is a function of both ion concentration and membrane potential that works without the use of metabolic energy. Upon activation of ion channels, the protein transmembrane helices move conformationally, opening a pore and allowing the passage of $10^{6}$ ions per second or greater (Fig. 1.7).

In electronics, the movement of the ion channel's lid is very similar to the dipole induced conformational movement of the nano-electro-mechanical relays (see Fig. 3.2). In theory, mechanical switches can eliminate the trade-off between power supply and leakage since they have essentially zero OFF current. Compared to the CMOS, these relays have high ON currents at smaller drain bias voltages and lower power dissipation. The gate in CMOS modulates the current flow by controlling the conductance of the channel, therefore CMOS requires optimal electrostatics. The charges are 
thermionically propagated from the source to the drain over a barrier in the presence of directional drain bias, limiting the steepest transition to $60 \mathrm{mV} /$ decade at room conditions. Like the ion channels, mechanical relays break this limit (see Fig. 3.12) because they mechanically amplify the modulation of the channel potential by the gate instead of amplifying the charge injection (charge multiplication). Thus under high gate bias, the entire channel film becomes fully conductive. The gate electrode is physically separated from the channel by a vacuum gap causing gate leakage to be zero. Opening the lid and allowing passive transport with gradient permits that many particles are able to move similarly to the ion channel for the expense of one.

\subsection{Dissertation Overview}

The remainder of this dissertation is organized as follows: Chapter 2 identifies a novel device, an electronic ratchet, which is capable of driving current in the absence of an applied drain bias. By using a time varying, asymmetric potential, we demonstrate the possibility of generating a net directional current from drift-diffusion processes of charge carriers. Chapter 2 further discusses connections between non-equilibrium electronic ratchets and sodium-potassium ion pumps. Chapter 3 presents the micro-scoping modeling of NEMFETs and profiles the significant characteristics of these devices that allow for their future use in electronics. Next, a discussion of connections between the ion voltage-gated channels with NEMFETs is presented. We focus on a comparison between the results of the empirical ion channel equations and the described novel modeling of NEMFETs. Finally, the dissertation concludes with Chapter 4, which summarizes all outcomes, findings, and contributions. A list of possible extensions of the project is included. Appendix I describes in detail how the body's nervous system communicates information between neurons in the form of the action potential spikes. Finally, we discuss how these action potentials are generated by two significant components: the ion pumps, and the voltage-gated channels, and describe their properties. 


\section{Chapter 2}

\section{Non-equilibrium Ratchets}

The largest impediment that sustained scaling faces is the thermal budget that arises from power dissipation in CMOS logic circuits [31, 47]. Although Biological systems (axonal networks) target very specific applications, as opposed to universal logic, they perform switching operations quite efficiently, albeit at lower speeds. The principles utilized by many biological systems are quite instructive, notably the use of analog encoding of signals (the inter-spike interval coding scheme) and the employment of strongly non-equilibrium power sources (the ionic pump) [48]. In this chapter, the electronic ratchets are modeled in a way similar to the Brownian motors. These motors use non-equilibrium noise to create directed motion [49,32,44]. The main goal of this chapter is to explore the distinct energy advantages of extending biological ratchet principles compared to solid-state electronics. Specifically, we investigate the shuttling of charges without a global drain bias under non-equilibrium conditions $[50,51,52]$, such as the hydrolysis of ATP over a finite noise frequency range. In Fig. 2.1, the connection between the electronic ratchet and the sodium-potassium pump is demonstrated. Both devices use a similar flashing asymmetric potential that moves a water molecule uphill against a gradient under non-equilibrium conditions.

This chapter identifies two distinct advantages of an electronic ratchets related to its device level energetics. First, by modeling the ratchet, we understand that the absence of a drain bias needed to create the global directionality that reduces the static dissipation. This is a distinct advantage in purely current-controlled logic (e.g. a Binary Decision Diagram or BDD), and the developed analytical equations show a deduction term in the dissipation. In a conventional CMOS incarnation, however, charging an output capacitor converts this current source into an effective drain voltage. At this 


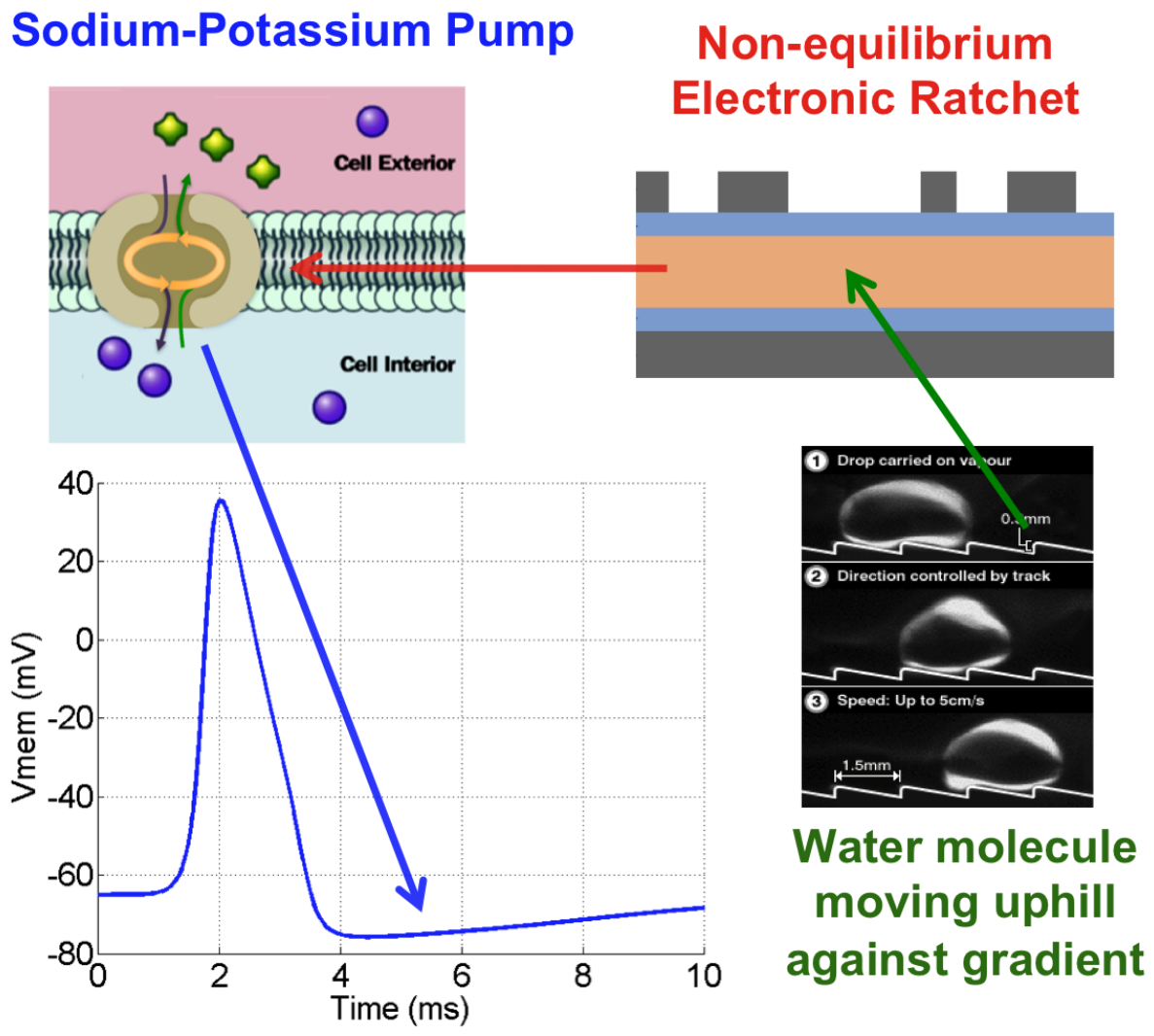

Figure 2.1: Ion pumps only operate with in the undershoot region of the action potential to replenish the voltage to the original conditions. Because the ion pumps and the electronic ratchets both work against an energy gradient by utilizing the non-equilibrium conditions, a connection can be made. Both devices use a flashing asymmetric potential similar to the uphill movement of a water molecule [4], to move particles or molecules against a gradient. In the ratchets, the asymmetry is created by the interdigitated electrodes, where as in the ion pumps the asymmetry is generated by the sticking and detachment of the ATP molecules.

stage, the second advantage of a ratchet is apparent: a voltage-controlled current source dissipates less energy when charging the capacitor and is amenable to adiabatic charging. Conventional schemes for adiabatic charging require precise timing information for signal synchronization. Because there is a wait time for each capacitor to charge adequately, such a timing requirement is not demanded of the electronic ratchets.

\subsection{Ratchet Physics: A Toy Model}

This chapter considers only a specific subset out of all possible ratchet types_ "flashing ratchets". Fig. 2.2 shows the basic operation of a flashing ratchet and how the particles will accumulate around a single potential minimum. This is how the ion pumps operate because the pump continuously moves individual ions from one end to the other end while flashing its potential. However, in a 


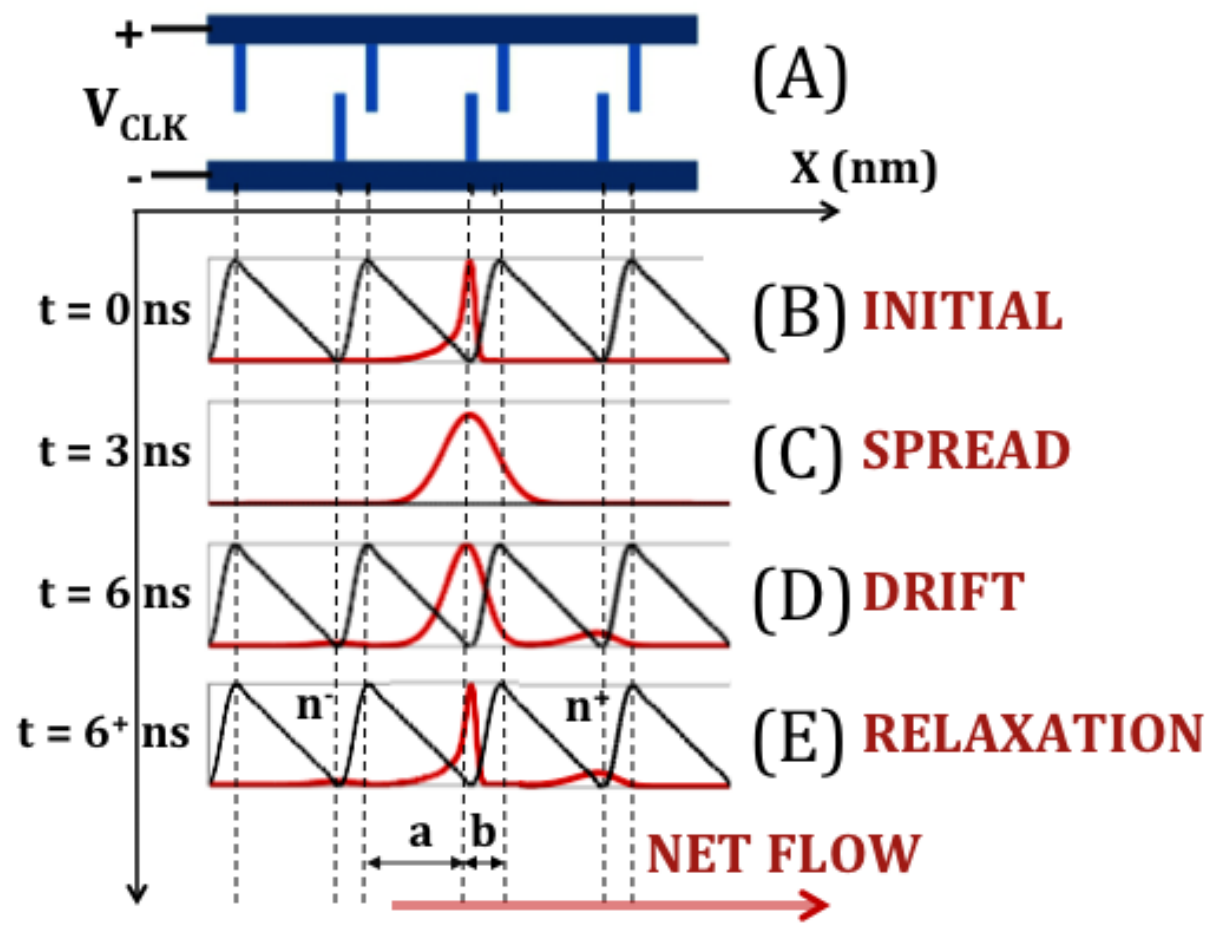

Figure 2.2: Figure above shows the carrier concentration with respect to the effects of the fluctuating asymmetric potential profile. Computing mechanism for a bistate quantum ratchet is simulated with $135 \mathrm{~nm}$ barrier length, $1.67 \mathrm{GHz}$ AC clock, and $20 \mathrm{kT}$ barrier height utilizing quantum flow and relaxation. (A) Interdigitated electrodes between two conductor planes creating the desired asymmetric local potential profile in the channel. (B) Initial carrier distribution (shaded area) when potential (dashed line) is "ON". The carriers are localized in the second well and can not diffuse to neither right or left well. (C) Distributed carriers when potential is "OFF". The carriers diffuse in both right and left directions equally. (D) Electron distribution when potential is again turned back on at the heated state. The red line $n^{-}$stands for the carriers overcoming the barrier peak on the left (back-flow), while $n^{+}$stands for those on the right (forward-flow). Since $n^{+}$is bigger than $n^{-}$, a net flow to the right results. (E) Relaxed stage at the end of each cycle, where the electron distribution $(\mathrm{n} / \mathrm{n} 0)$ is reseted to the initial equilibrium solution to cool the system down.

real electronic device, the particles will be distributed equally to every minima. A periodic potential with built-in local asymmetry is created by a sequence of interdigitated electrodes (see Fig. 2.2A). When the barriers are fully raised, the carriers injected into the channel localize around the potential minima (see Fig. 2.2B). When the applied AC clock lowers the barriers (see Fig. $2.2 \mathrm{C}$ ), the carriers spread out in both left and right directions, driven by thermal diffusion in the classical limit and by wave packet evolution in the quantum limit, driven by the difference in phase velocities of the individual Fourier components. When the potential is turned back on again, the carriers drift down to the nearest valleys (see Fig. 2.2D). Owing to the local asymmetry of the potential profile, there will be more carriers that have crossed the top of the barriers into each valley compared to the barrier sitting further, because the diffusion occurs when the barriers are down. In 
other words, there is a progressive space-time averaged unidirectional movement of charges even in the absence of a source-drain bias, driven simply by the non-equilibrium signal supplied by the clock.

The action of a ratchet can be described as a clock-driven current source that can be used to build an open-circuit voltage, $V_{O C}$, across a capacitor in a logic circuit. As the voltage builds on the capacitor, it creates a back-flow until the reverse bias current cancels the ratchet current upon complete charging of the capacitive load (In the ion pumps, the ratchets stops operating once enough ions are moved across the membrane to reestablish the resting potential back, in order to prepare the axon ready for another excitation.). A large open-circuit voltage allows the electronic ratchet to drive the next stage, but this efficiency needs to be counter weighed against the corresponding energy dissipated and the charging delay associated with building the voltage.

\subsubsection{Asymmetric Potential Creation}

Developing the asymmetric saw tooth potential on a semi-conducting channel material has been demonstrated in number of experiments by many different methodologies[53, 54, 55, 56]. In [53], authors have used different work function on electrode pairs, which were separated by $200 \mathrm{~nm}$, to

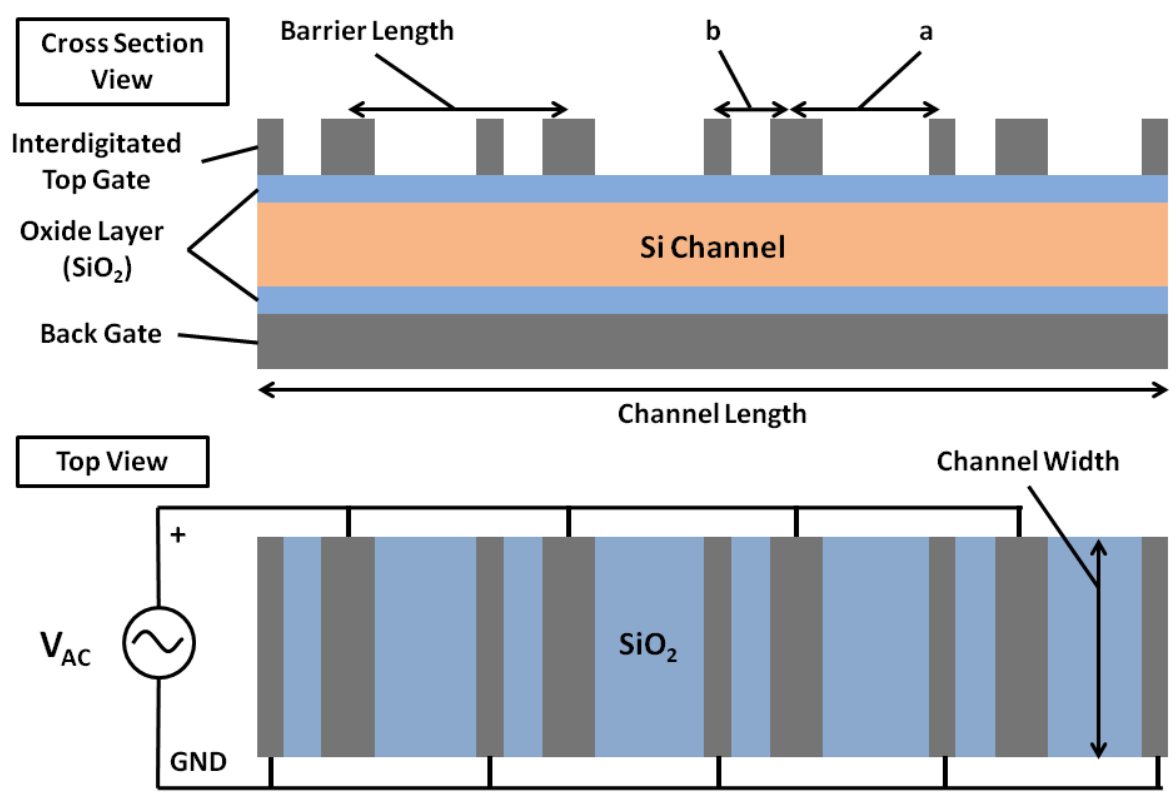

Figure 2.3: Prototypical geometry of a ratchet consisting of an interdigitated top gate, which creates the asymmetric time varying potential, and a back gate, which determines the initial number of carriers in the channel by shifting the Fermi level. The current driven by the top gate is used to build up voltage, which can be used to back gate the next cascaded ratchet in the logic architecture. This asymmetric potential profile is very similar to the ion pump potential profile shown in Fig. 1.6 

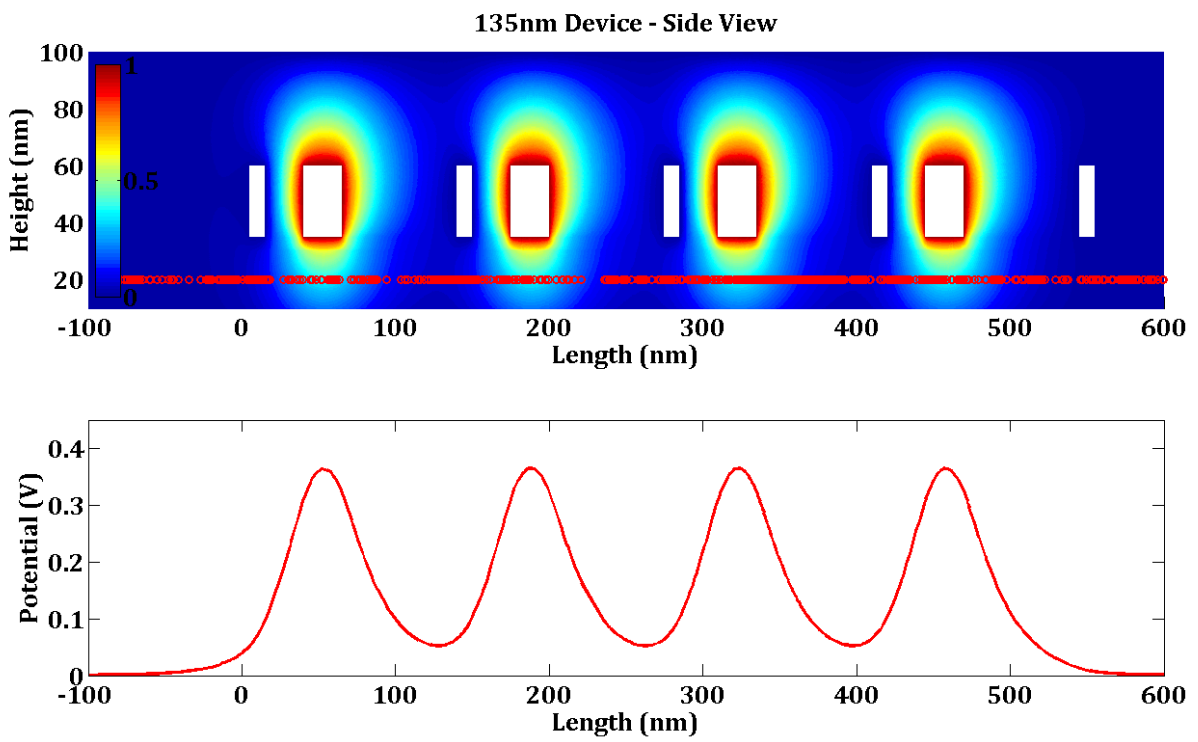

Figure 2.4: Simulation of the potential profile for a ratchet with $135 \mathrm{~nm}$ barrier length using the Laplace tool box in MATLAB (PDEtool). The positive electrodes are $25 \mathrm{~nm}$ in width whereas the grounded electrodes are $10 \mathrm{~nm}$ in width to increase the asymmetry. The $a / b$ separation factor between the electrodes is 3 . The lower figure shows the Laplace potential through the center of the Si channel.

generate the asymmetric potential profile. They managed to fabricate 6 asymmetric barriers on a 2.5 $\mu \mathrm{m}$ (width) by $10000 \mu \mathrm{m}$ (length) transistor channel with $70 \mathrm{mV}$ surface potential difference. In [54], investigators have showed that in order to achieve asymmetric potential with an in-plane interdigitated comb-drive, the minimum engagement length of the comb must be twice the separation gap between two adjacent combs. By using the interdigitated electrodes in [55], authors have demonstrated a direct motion of particles when voltage difference is applied between the adjacent electrodes. In [56], instead of being asymmetric in space (ratchet potential), investigators varied the time-asymmetry of the drive and experimentally showed increases and decreases in the density of magnetic flux at the center.

Fig. 2.3 shows a possible implementation of a ratchet-based switch. The design has a dual gated device with a top and a back gate capacitor. The top gate, consisting of interdigitated metal plates, creates a spatially asymmetric potential - obtained by solving Laplace's equation (Fig. 2.4). An AC signal applied to these plates creates a clock that periodically raises and lowers the potential barriers. The ratchet mechanism (described in the next section) creates a net non-zero DC current averaged over space and time, and this current progressively builds up a charge on the back gate capacitor. This flashing movement of the potential is very similar to the potential profile generated in the ion 
pumps, by the dipoles sticking and unsticking to the surface (see Fig. 1.6). The back gate capacitor represents the input gate to the next ratchet in series (not shown), shifting its Fermi energy and turning it on so that the second ratchet can start shuttling charges. A suitable layout of a ratchet array can then realize a NAND, a NOR or other generic Boolean logic gates.

\subsubsection{Approximate Quasi-Analytical Result}

Using the diffusion equation[57], near the valley at $x_{0}$, the potential profile can be approximated as

$$
U(x) \approx U\left(x_{0}\right)+\frac{\left(x-x_{0}\right)^{2}}{2} U^{\prime \prime}\left(x_{0}\right)
$$

where dash represents a 1-D spatial derivative. The corresponding equilibrium initial carrier distribution becomes

$$
N(x) \propto \exp [-U(x) / k T]=n_{0} \exp \left[-\frac{\left(x-x_{0}\right)^{2}}{2 \sigma_{0}^{2}}\right]
$$

where $\sigma_{0}=\sqrt{k T / U^{\prime \prime}\left(x_{0}\right)}$ is the standard deviation of Gaussian distribution and $U\left(x_{0}\right)$ is set to be zero for convenience. After one computing cycle of the electronic ratchet (see Fig. 2.2), the net carrier density difference between two neighboring potential wells is

$$
\Delta N=n_{0} S l_{\text {barr }}\left[\operatorname{erfc}\left(\frac{b}{\sqrt{2 \sigma_{0}^{2}+4 D t_{o f f}}}\right)-\operatorname{erfc}\left(\frac{a}{\sqrt{2 \sigma_{0}^{2}+4 D t_{o f f}}}\right)\right]
$$

where $\operatorname{erfc}$ is the complementary error function, and $n_{0}=N_{c} \exp \left(-\frac{E_{f}-E_{c}}{k T}\right)$ is the initial carrier density in the semiconducting channel, $N_{c}$ is the effective density of states, $E_{f}$ is the Fermi level, $E_{c}$ is the conduction band level, $D$ is the diffusion constant, $S$ is the channel cross-sectional area, $l_{b a r r}$ is the length of the potential barrier, $t_{o n}$ is the duration of the "ON" part of the clock when the barrier is raised, $t_{o f f}$ is the "OFF" time period when the barrier is lowered, $a$ and $b$ are the asymmetric physical lengths defined in Fig. 2.2D. This expression also assumes that the drift time is smaller than $t_{o n}$, i.e. the barrier is raised long enough for the charges to drift into the valley, whereupon phonons rapidly relax their energy. Finally, the net space- and time-averaged current under zero reverse bias becomes

$$
I_{0}=\frac{q \Delta N}{t_{o n}+t_{o f f}}
$$

It is clear that the current arises because of the asymmetry $(a>b)$, driven by the clock frequency related to $t_{o n, o f f}$. 


\subsubsection{Simulating a Quantum Ratchet}

The quantum flow of electrons in the electronic ratchet involves solving the time-dependent oneelectron Schrodinger equation

$$
i \hbar \frac{\partial \psi}{\partial t}-H \psi=0
$$

where $H$ is the time-dependent Hamiltonian matrix describing the channel. The Hamiltonian is described using the 1-D finite difference tight-binding formula

$$
H_{n, m}=\left[U_{n}+2 t_{0}\right] \delta_{n, m}-t_{0} \delta_{n, m+1}-t_{0} \delta_{n, m-1}
$$

where $t_{0} \equiv \hbar^{2} / 2 m^{*} a^{2}$ depends on the grid size $a$ and the effective mass $m^{*}$. To calculate the ballistic non-equilibrium flow in a drain-driven device, the self-energy matrices for injection and removal is normally added in and coupled with bias-separated contact Fermi-Dirac distributions [58]. For the electronic ratchet however, flow is generated simply by the time-dependence in $H$. In order to visualize this short-circuit current, the model simply incorporates periodic boundary conditions for the shuttling of charges. The initial state of the particles is obtained from the eigenvectors $\{\alpha\}$ and eigenvalues $E_{\alpha}$ of the matrix $[H]$ at the time instant $t=0$, while the subsequent evolution of the wave-functions is obtained solving the Crank-Nicholson approximation for its efficiency and simplicity in computing

$$
\psi_{t+\Delta t}=\left[1-\frac{i H \Delta t}{2 \hbar}\right]^{-1}\left[1+\frac{i H \Delta t}{2 \hbar}\right] \psi_{t}
$$

In addition to a local asymmetry and an energy source, the ratchet requires dissipation to generate a "reset" at the end of each cycle. A purely ballistic quantum evolution, described above, does not have an inbuilt mechanism for relaxing the charges. As a result, the simulations show that the process of continuously pumping energy into the ratchet from an AC field causes the carriers to heat up and eventually fly off the barriers. Thus, at the end of each AC cycle, a way to remove the excess energy is needed. Coupling the electrons with substrate phonons would remove that energy. Rather than modeling this complex behavior, this relaxation is capture by using a "one-shot" procedure, where at the end of each clock cycle, the electron distribution is reseted to the initial equilibrium solution. The energy difference, between the equilibrium eigenvalue and that reached at the end of the $\mathrm{AC}$ cycle, is the final dissipation value.

The time-dependent potential, $U_{n} \equiv U\left(x_{n}\right)$, samples the asymmetric barrier shape, which is modeled by $U(x)=x^{2}\left(1-x^{7}\right)$. Periodic boundary conditions are invoked by setting $H_{1, N}=H_{N, 1}=$ 
$-t_{0}$, while open boundary conditions require self-energy matrices with $\Sigma_{1}(1,1)=\Sigma_{2}(N, N)=-t_{0} e^{i k a}$. While periodic boundary conditions suffice to see the shuttling of charges, and in addition provide analytical simplicity for a closed circuit, there is a danger of tails of the wave-function escaping the drain and re-injected from the source towards a valley and self-interfering, an issue that open boundary conditions do not have. This becomes a bigger issue if the charges are allowed to build up at the end for an open-circuit, whereupon the periodic boundary conditions cannot even be justified. Consequently, open boundary conditions are used for the latter half of the chapter (see section 3.2.5).

\subsubsection{Simulating a Classical Ratchet}

Classically, simulations keep track of the charges rather than wave-functions, and evolve them by the Newtonian drift-diffusion equation

$$
\frac{\partial N}{\partial t}=-\frac{\partial(\mu \xi N)}{\partial x}+D \frac{\partial^{2} N}{\partial x^{2}}
$$
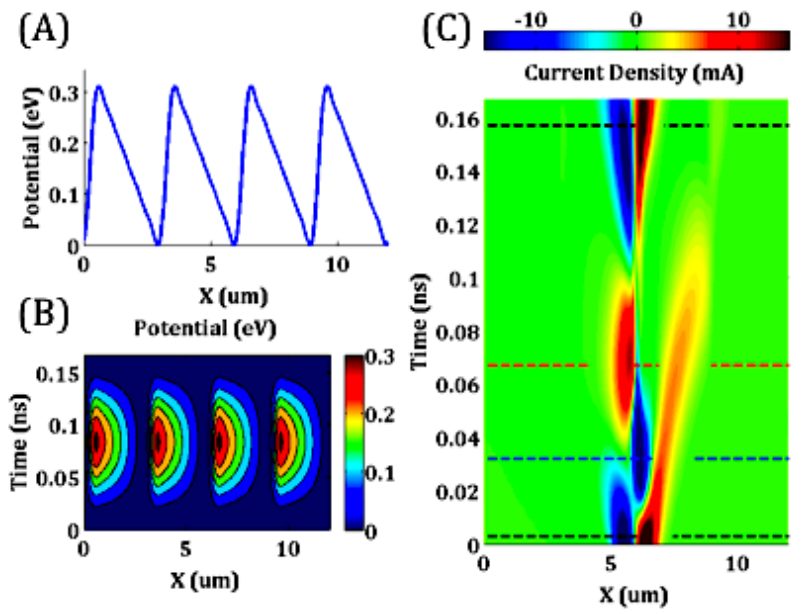

(D)

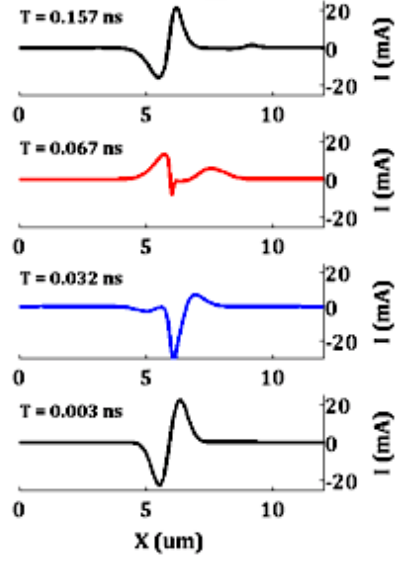

Figure 2.5: (A) Spatiotemporal ratchet potential, with a prominent spatial asymmetry with four barriers. (B) Contour-plot of this ratchet potential progressively turned ON between 0.02 and 0.08 ns and then turned OFF after $0.8 \mathrm{~ns}$. (C) Colormap of the current density over this time (blue is negative, red is positive, and green is zero). As seen from the figure, the drift segment of the current during the potential upcycle is actually two phased, showing a quick initial drift down the steeper slope (thin, localized dark blue to cyan), followed by a slower drift of a larger number of particles (hence, a net positive current) down the smaller slope shown in spreading red to yellow. The current density plots shown in (D) are the snapshots at time instances marked by the horizontal dashed lines in (C). During down cycle, the back and forward propagating currents (blue and red, near $t=0.157$ ns) are comparable as the diffusion process is symmetric in space whereas when potential is turning up, near $t=0.032 \mathrm{~ns}$ back-flow dominates and at $t=0.067 \mathrm{~ns}$ the forward-flow is more prominent. Thus, the net current flow proceeds with a two-phased drift, supplanted gradually by a symmetric diffusion. 
(A)

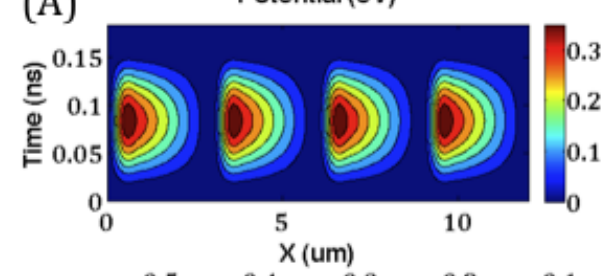

(B)

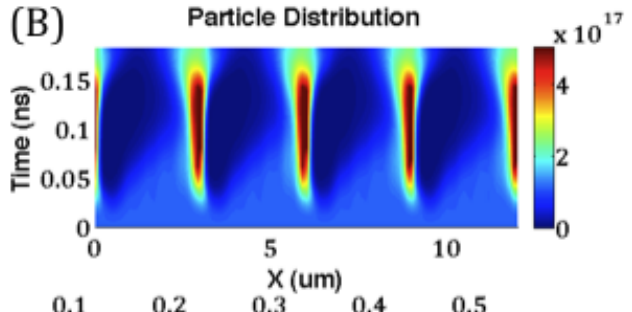

$\frac{1}{i m A}$

(D)

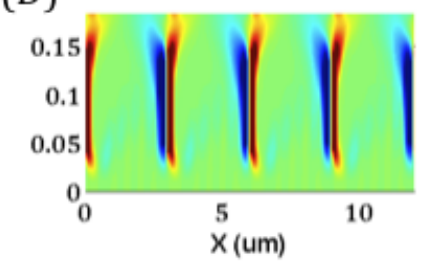

(E) Total Current Density (mA)

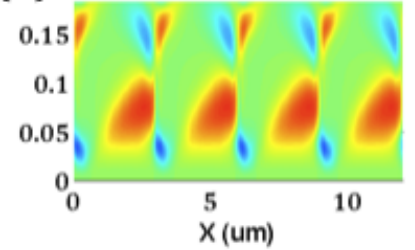

Figure 2.6: (A) Asymmetric ratchet potential changes with time and space. (B) Particles are evenly distributed (localized) in bins when the potential is raised and how the particles move to the right with the oscillating top gate (the blue area in between localized bins shows the pathway of particles moving right). (C)-(E) Total, drift, and diffusion current densities. One can see that drift current opposes the diffusion current at all the times, but their magnitudes are different, thus resulting in a net flow to the right. As discussed in the previous sections, the second phase of the two-part drift current shown in yellow moves the particles to right, then the diffusion current spreads the particles evenly right and left.

where $N=N(x, t)$ is the carrier distribution, $\xi=\xi(x, t)$ is the electric field, $\mu$ is the carrier mobility, and $\mathrm{D}$ is the diffusion constant. The triangular potential affects the carriers through the electric field given by the equation

$$
\xi=-\frac{d U}{d x}
$$

where $U$ is the spatially varying potential profile across the ratchet. The current can be calculated from $\mathrm{N}$ as

$$
J=q v N+q D \frac{\partial N}{\partial x}
$$

where $v=\mu \xi$ and $q$ is the electric charge. Fig. 2.5A,B show how the potential is varied with space and time. Fig. 2.5C demonstrates how the total current density is generated by the movement of electrons and has a net positive current flow to the right. The Gaussian distributed particles initially localize in the second well $(\mathrm{L} \approx 6 \mu \mathrm{m})$ and start to diffuse both sides at $t=0.003 \mathrm{~ns}$ equally. As the potential starts to turn on between 0.02 and $0.08 \mathrm{~ns}$, a small amount of particles (due to asymmetry of potential) quickly drift down the steeper slope (thin dark blue to cyan) followed by a slower, positive drift movement of a larger group of particles (red/orange to yellow) resulting in a net positive forward-flow. At $t=0.157 \mathrm{~ns}$, the potential is lowered and the diffusion starts, helping the two-phase drift current. As shown in the current figures of Fig. 2.6E, the net flow of particles 
is caused mostly by the drift current (see Fig. 2.6C), not by the diffusive current, which is almost symmetrical in both directions (see Fig. 2.6D).

The boundary condition at the contacts is set to describe carrier recombination in the relaxation time approximation

$$
\left.\frac{d N}{d t}\right|_{\text {end }}=-\frac{N_{\text {end }}}{\tau}
$$

where $\tau$ is the time it takes for the electrons to transfer from the semiconductor to the capacitor. In these simulations, copper is selected as the capacitive material for the back gate of the next ratchet (see Table 2.1) [59]. This boundary condition occurs because of the interface between the ratchet channel and contact, and it usually limits the amount of charge, which can flow in and out of the capacitor. However, in the simulations, the charge extraction rate for copper was fast enough to allow all charge carriers to (dis) charge the capacitor. Therefore, the contact interface did not limit the current flow between the capacitor and the ratchet.

In order to capture the charging process of the load capacitor, the number of charges accumulating on the capacitor was calculated as $Q=J_{\text {end }} S \Delta t$, where $J_{\text {end }}$ represents the current density crossing the boundary at the end of each time step, $S$ is the channel cross sectional area, and $\Delta t$ is the simulation time step. Using a time step one can calculate the voltage increase as $V=Q / C$, where $C$ is the load capacitance. Then, the model superimposes a backward voltage varying linearly from 0 to $V$ across the ratchet (ideally, by solving Poisson's equation, but simplified in the treatment here), and add it to the sawtooth potential to give a tilted asymmetric potential. Finally, the electric

Table 2.1: Simulation Parameters for Drift-Diffusion

\begin{tabular}{|c|c|}
\hline Barrier Length $(L)$ & $3 \mu \mathrm{m}$ \\
Asymmetry $(a-b)$ & $2.25 \mu-0.75 \mu \mathrm{m}$ \\
Channel Thickness $(t)$ & $20 \mathrm{~nm}$ \\
Channel Width $(W)$ & $6 \mu \mathrm{m}$ \\
Oxide Thickness $\left(t_{o x}\right)$ & $5 \mathrm{~nm}$ \\
Top Gate AC Clock & $0.39 \mathrm{~V} @ 6 \mathrm{GHz}$ \\
Diffusion Constant $(D)$ & $3.62 \times 10^{-3} \mathrm{~m}^{2} \mathrm{~s}^{-1}$ \\
Si Eff. Den. of States $\left(N_{c}\right)$ & $3.23 \times 10^{25} \mathrm{~m}^{-3}$ \\
$\eta=\left(\frac{E_{c}-E_{f}}{k T}\right)$ & 3 \\
Max. Ratchet Barrier Height $(\mathrm{in} k T / q)$ & 15 \\
Capacitor Escape Rate (for $\mathrm{Cu})(\tau)$ & $1.786 \times 10^{-14} \mathrm{~s}$ \\
\hline
\end{tabular}




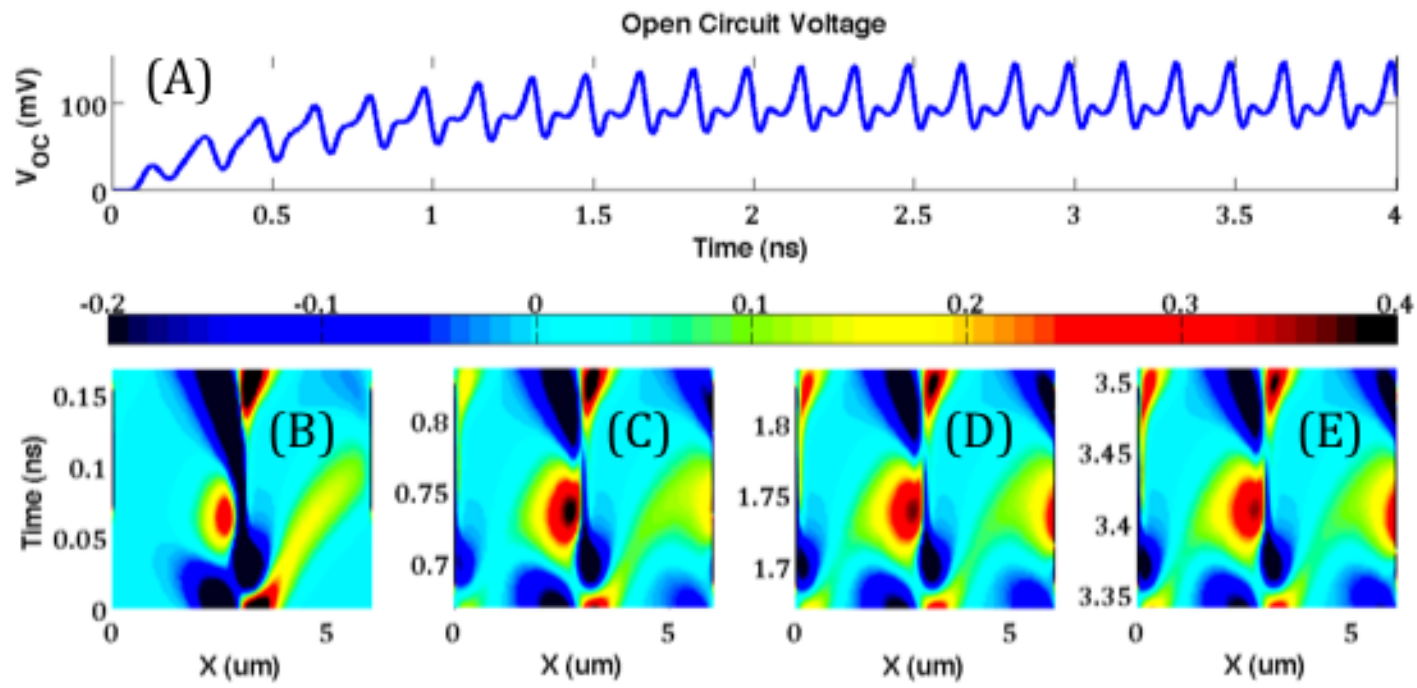

Figure 2.7: (A) Drift-diffusion simulation of the electronic ratchet showing the charging of a load capacitor. After 11 clock cycles the particles are distributed equally among all the barriers and there is a net forward-flow of current to the right. The two-phase drift current is less prominent as the $V_{O C}$ is built-up, but still exists as seen in the (B)-(E) (darker red colors start changing to orange/yellow colors as the $V_{O C}$ increases). As the particles reach the right side $(\mathrm{L}=6 \mu \mathrm{m})$, they are ejected by the system and charge a localized back gate capacitor to a $V_{O C}$ of $\approx 145 \mathrm{mV}$.

field is recalculated and reiterated by 2.8 until the capacitor is charged under open bias and the net space-time-averaged current vanishes (shown in Fig. 2.7). Note that there is still dynamic current flow and associated dissipation.

\subsubsection{Calculating the Open-Circuit Voltage}

Charging of the load capacitor increases the voltage on load side of the ratchet, resulting in a tilted potential growing with time across the device. The resulting reverse current partially cancels out the directional current from the ratchet. This part of the operation of the electronic ratchets is slightly different then the ion pumps, rather than the potential difference, the pumps stop when the ionic difference is reached. Eventually, the capacitor reaches a critical voltage, called the open-circuit voltage $\left(V_{O C}\right)$ (or the resting potential in the ion pumps) when the reverse current equals the current generated by the electronic ratchet, averaged over space and time. It is therefore important to extract the open-circuit voltage in order to understand the drivability of the ratchet device for logic.

Let us assume that the time dependent current through the ratchet in one operation cycle can be given by $I(t)$. Therefore, the current generated by the ratchet after one clock cycle is

$$
I_{0}=\frac{1}{t_{o n}} \int_{t_{o f f}}^{t_{o n}+t_{o f f}} I(t) d t=\frac{Q}{t_{o n}}
$$


where $Q$ is the total charge placed on the load capacitor. The voltage generated on the load capacitor after one cycle is $V_{0}=Q / C$. This, in turn, creates a reverse current of $I_{\text {rev, } 0}=V_{0} / R$, where $R$ is the resistance of the ratchet channel (in general, a nonlinear resistance, which must be calculated self-consistently). If the space-time averaged current pumped by the ratchet is assumed to remain constant, the total current generated by the ratchet during the next cycle given by

$$
I_{1}=\frac{Q}{t_{\text {on }}}-I_{\text {rev }, 0}
$$

This way, the voltage on the capacitor after $N$ cycles is

$$
\begin{aligned}
V_{N} & =\frac{1}{C} I_{N} t_{\text {on }}+V_{N-1} \\
& =\frac{1}{C}\left[\frac{Q}{t_{\text {on }}}-\frac{V_{N-1}}{R}\right] t_{\text {on }}+V_{N-1} \\
& =\frac{Q}{C}+\left(1-\frac{t_{\text {on }}}{R C}\right) V_{N-1} \\
& =\frac{Q}{C}\left[\frac{1-\left(1-\frac{t_{\text {on }}}{R C}\right)^{N}}{\frac{t_{\text {on }}}{R C}}\right]
\end{aligned}
$$

Therefore, the open-circuit voltage can be defined as

$$
\begin{aligned}
V_{O C} & \equiv \lim _{N \rightarrow \infty} V_{N}=\frac{Q}{t_{\text {on }}} R \\
& =\frac{l_{\text {barr }}}{q n_{0} \mu S} \cdot \frac{1}{t_{\text {on }}} \int_{t_{\text {off }}}^{t_{\text {on }}+t_{\text {off }}} I(t) d t
\end{aligned}
$$

It is important to note that the output voltage is only indirectly dependent on the input voltage. The input voltage from the back gate increases the number of carriers in the channel, and thus the magnitude of $V_{O C}$. However, since the top gate is always oscillating, it does not influence the input that determines the output. The input is decoupled from the charging process of the output, which means that the ratchet back gate is adiabatically charged without placing any timing constraints on the input.

If we now apply (2.15) to (2.4), we derive the following $V_{O C}$ for the Gaussian distribution:

$$
V_{O C}=\frac{l_{\text {barr }}^{2}}{2 \mu\left(t_{\text {on }}+t_{\text {off }}\right)}\left[\operatorname{erfc}\left(\frac{b}{\sqrt{2 \sigma_{0}+4 D t_{o f f}}}\right)-\operatorname{erfc}\left(\frac{a}{\sqrt{2 \sigma_{0}+4 D t_{o f f}}}\right)\right]
$$

For the simulations, the parameters listed in Table 2.1 are used. The parameters are conventional 
material properties of silicon for the channel and silicon dioxide for the oxide [57]. Furthermore, we derive device parameters, such as back gate capacitance, from the dimensions and structures shown in Fig. 2.3.

In Fig. 2.7, the charging of the load capacitor is demonstrated. According to the drift-diffusion simulations, the open-circuit voltage saturates to $\approx 145 \mathrm{mV}$, which is in a relatively good agreement with the analytic value of $\approx 125 \mathrm{mV}$ derived by using the parameters from Table 2.1 in (2.16). From the 3-D figure showing different time cycles in Fig. 2.7A, we can see that as the forward-flow of electrons decreases with time, $V_{O C}$ builds up. In the Fig. $2.7 \mathrm{~B}$ at $\mathrm{t} \approx 0.05 n s$ and $\mathrm{x} \approx 5 \mu \mathrm{m}$, there is net positive current shown in yellow, but as the $V_{O C}$ builds, this positive current becomes less prominent and disappears as shown in Fig. 2.7E. The space-time averaged net-current becomes zero.

\subsection{Logic with Ratchets}

\subsubsection{Static Power in CMOS Versus Ratchet}

Static power dissipation in CMOS circuits is caused by a direct current from the power source $\left(V_{d d}\right)$ to ground (see Fig. 2.8A). In an ideal CMOS circuit, there is no static dissipation because its complementary nature ensures that for each turned ON PMOS, there exists a turned OFF NMOS blocking a direct path to ground, or vice versa. However, in real circuits, MOSFETs are not completely turned $\mathrm{OFF}$ and there exist leakage currents, which traverse from $V_{d d}$ to ground-creating static power dissipation. In modern CMOS circuits, leakage power is a significant problem, which usually accounts for a large portion of all power dissipation [60].
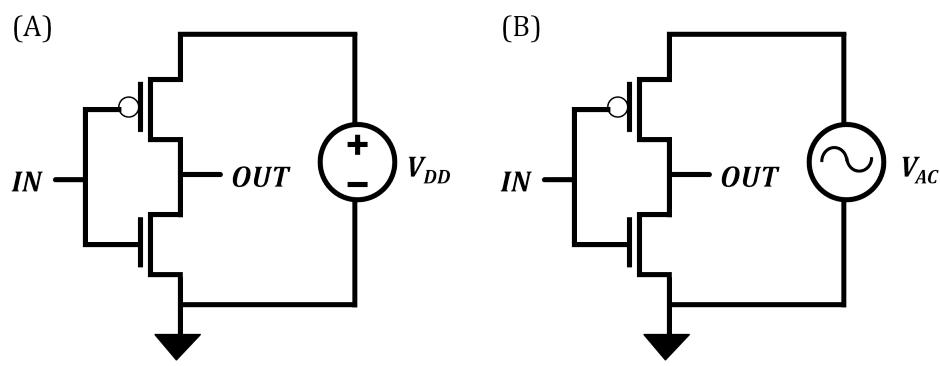

Figure 2.8: Conventional versus adiabatic charging. (A) CMOS circuits are conventionally charged using a DC voltage source. (B) By using a low frequency AC voltage source, the capacitor can be charged while dissipating less energy [8]. 
The electronic ratchet, on the other hand, can avoid this issue altogether. Rather than using a DC power source to generate a current, the ratchet uses a time varying asymmetric potential to create a net current. Therefore, the ratchet can drive current without a source to drain bias. Since there are no DC power sources, the ratchet can, in principle, be used to design circuits with no static dissipation.

\subsubsection{Dynamic power in CMOS Versus Ratchet}

In CMOS logic circuits, dynamic power dissipation results from the charging and discharging of load capacitors. Take the inverter in Fig. 2.8A for example. When the inverter has an input of '0', the load capacitor is charged to $Q=C V_{d d}$. Therefore, the energy dissipated is given by:

$$
\begin{aligned}
E_{\text {diss }} & =E_{\text {source }}-E_{\text {cap }} \\
& =\int_{0}^{Q} V_{d d} d Q-\int_{0}^{Q} V d Q \\
& =C V_{d d}^{2}-C \int_{0}^{V_{d d}} V d V \\
& =\frac{1}{2} C V_{d d}^{2}
\end{aligned}
$$

This energy is dissipated in the form of heat due to the effective resistance of system. The dissipated power is given by the equation $P=\frac{1}{2} C V_{d d}^{2} f$, where $f$ is the operating frequency of the CMOS circuit. An alternative method of charging, which reduces the dynamic dissipation is using a voltage-controlled current source similar to the electronic ratchet. It will be shown in Section III-C that a load capacitor charged through a voltage-controlled current source is

$$
E_{\text {ratchet }}=\frac{1}{2} C V_{O C}^{2}\left(\frac{R}{R+V_{O C} / I_{m}}\right)
$$

where $I_{m}$ is the maximum current through the ratchet when the load capacitor is uncharged (i.e., the short-circuit current), $V_{O C}$ is the final open-circuit voltage across the ratchet, and $\mathrm{R}$ is the external interconnect resistance. While $V_{O C}$ effectively plays the role of a drain bias that develops across the ratchet, with a magnitude that is set by the desired ON-OFF ratio, the added term in the denominator helps shave off some of the dynamic power dissipation.

Another way to further reduce this dissipated energy is a method known as adiabatic charging [61]. Fig. 2.8B shows an example of an adiabatic CMOS circuit, where the DC power source has 
been replaced by an oscillating signal, known as a clock. Now let's assume that the input is '0' as the clock begins to ramp up. If it takes $t=\Delta T$ for the load capacitor to charge to $V_{d d}$, then the energy dissipated during the charging process can be given as:

$$
\begin{aligned}
E_{\text {diss }} & =\eta P \Delta T \\
& =\eta I^{2} R \Delta T \\
& =\eta\left(\frac{C V_{d d}}{\Delta T}\right)^{2} R \Delta T,
\end{aligned}
$$

where $\eta$ is a factor, which is dependent on the shape of the waveform of the clock [62]. Notice that by increasing $\Delta T$, one can reduce the dissipated energy indefinitely. However, one of the drawbacks of adiabatic CMOS is that it requires timing information for the inputs in order to maintain adiabatic operation. For example, it is important that to turn on the transistor when the clock is ramping up. If the transistor is turned on when the clock was at its maximum voltage, then there would have been a large voltage drop across the effective resistance of the circuit, leading to non-adiabatic dissipation.

In the electronic ratchet, the time-varying asymmetric potential acts as the clock in an analogous fashion as the adiabatic CMOS. However, as showed in Section II, the input of the ratchet is decoupled from the charging process of the output. This allows us to take advantage of the adiabatic charging without imposing timing constraints on the inputs. In Fig. 2.9, a circuit symbol of electronic ratchet is presented showing the input and output ports.

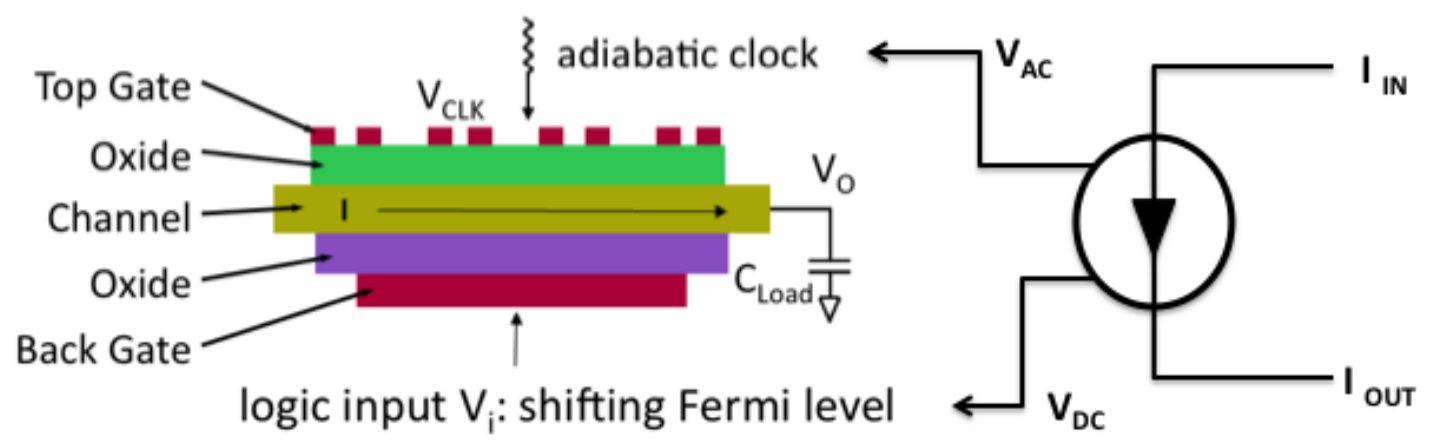

Figure 2.9: Structure of a single ratchet and its circuit representation, showing the input and output ports of the ratchet modeled a voltage-controlled current source. 

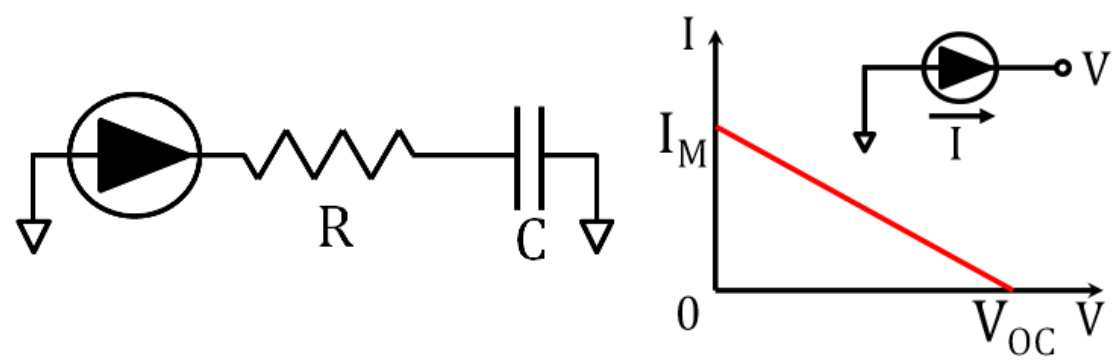

Figure 2.10: Equivalent circuit of the charging behavior of the ratchet. The ratchet acts as a voltage-controlled current source, where current is a function of the voltage on the capacitor. The current is at a maximum $I_{m}$ initially when the capacitor is uncharged. As the voltage builds up, the reverse current decreases the net current, until the capacitor reaches the open-circuit voltage, $V_{O C}$ when there is a zero net current.

\subsubsection{Capacitive Charging Using a Current Source}

Consider a simple circuit model, in which an electronic ratchet charges a capacitor, as shown in Fig. 2.10. The $\mathrm{I}-\mathrm{V}$ characteristic of the electronic ratchet is also shown and can be written by using the Kirchoff's equations to describe this circuit as

$$
\begin{aligned}
I & =\frac{V-V_{c}}{R} \\
I & =-\frac{I_{m}}{V_{O C}} V+I_{m} \\
I & =C \frac{d V_{c}}{d t}
\end{aligned}
$$

where $I$ is the current, $V$ is the voltage of the node between the electronic ratchet and the resistor and $V_{c}$ is the voltage on the capacitor.

From the (2.21)-(2.22), the voltage on the capacitor, $V_{c}$, and current, $I$ can be solved as

$$
\begin{aligned}
V_{c} & =V_{O C}\left(1-e^{-t / R^{\prime} C}\right) \\
I & =\frac{V_{O C}}{R^{\prime}} e^{-t / R^{\prime} C}
\end{aligned}
$$

in which $R^{\prime}=R+V_{O C} / I_{m}$. Therefore, the energy charged into the capacitor is

$$
E_{c a p}=\int_{0}^{V_{O C}} C V_{c} d V_{c}=\frac{1}{2} C V_{O C}^{2}
$$


and the dissipated energy on the resistor is

$$
\begin{aligned}
E_{R} & =\int_{0}^{\infty} I^{2} R d t \\
& =\int_{0}^{\infty}\left(\frac{V_{O C}}{R^{\prime}}\right)^{2} e^{-2 t / R^{\prime} C} R d t \\
& =\frac{C V_{O C}^{2}}{2} \frac{R}{R^{\prime}} \\
& =\frac{C V_{O C}^{2}}{2} \frac{R}{R+\frac{V_{O C}}{I_{m}}}
\end{aligned}
$$

From this equation, the following observations are made. In a ratchet, the load capacitor is charged to $V_{O C}$ rather than $V_{d d}$. Therefore, the ratchet energetics are comparable to CMOS circuits with extremely low drain-source voltages. Furthermore, the ratchet introduces an extra resistance term, $V_{O C} / I_{m}$, which decreases the energy dissipation further. For the simulations (see Section 3), the channel resistance was $1.39 \mathrm{k} \Omega$ compared to the extra resistance term of $2.70 \mathrm{k} \Omega$. Therefore, the unique charging method of the ratchet makes it intrinsically less dissipative than conventional CMOS logic.

\subsubsection{Adiabatic Charging}

A more accurate circuit model for the ratchet is developed (see Fig. 2.11) to describe its energetics during adiabatic charging. An LC circuit is used to represent the oscillating clock for the top gate. This clock charges up the top gate capacitor, which represents the charging or discharging of the asymmetric ratchet potential. Because the circuit is operating at the natural frequency, we are able to adiabatically transport electrons in the channel, thus reducing the energy dissipation of the circuit.

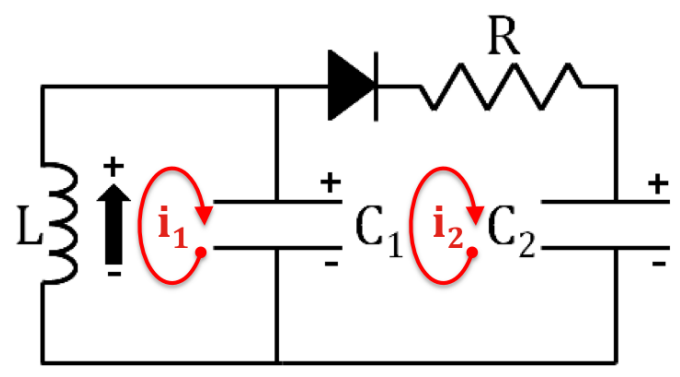

Figure 2.11: Circuit model for the ratchet. An adiabatic clock is modeled using an inductor $\mathbf{L}$ and the top gate capacitor $C_{1}$. A (virtual) diode is used to capture the unidirectional current (ratchet) which drives charging of the load capacitor $C_{2}$. 
The asymmetry of the ratchet potential provides directionality to the electrons and gives a net positive current. This asymmetry is captured by a (virtual) Schottky diode, which allows current to flow easily in one direction while acting as a rectifier in the opposite direction. The diode parameters are selected by calibrating with the drift-diffusion simulation. The barrier height is selected as 15 $k T / q$ as in the simulations and the $I_{0}$ is selected as $1 \times 10^{-15} A$ to match the results. The diode also acts as the voltage-controlled current source described in Fig. 2.10. This current is used to charge the load capacitor, which acts as the back gate capacitor of the next ratchet in a cascaded logic architecture (see Fig. 2.13).

The charging process can be seen in the following analysis. We treat the diode as a short-circuited element when there is a positive current running through it, and as an open-circuit when the current is negative. During the time when the diode is short circuited, we have the following Kirchoff's equations

$$
\begin{array}{r}
L \frac{d i_{1}}{d t}-\frac{Q_{1}}{C_{1}}=0 \\
\frac{Q_{1}}{C_{1}}-R i_{2}-\frac{Q_{2}}{C_{2}}=0
\end{array}
$$

where $i_{1}$ and $i_{2}$ are the currents in the two separate loop circuits. For (2.26), we assume that the charge on the top gate capacitor, $C_{1}$, is exclusively determined by the current generated by the inductor. This is a valid assumption if the top gate capacitance is much larger than the back gate capacitance (see Table 2.2), thus acting as an energy "tank" [63].

We first solve (2.26), and apply the boundary condition $d Q_{1} /\left.d t\right|_{t=0}=-I_{0}$

$$
\begin{array}{r}
\frac{d^{2} Q_{1}}{d t^{2}}-\frac{Q_{1}}{L C_{1}}=0 \\
Q_{1}(t)=-\frac{I_{0}}{\omega_{L}} \sin \omega_{L} t
\end{array}
$$

Table 2.2: Parameters for Circuit Simulations
\begin{tabular}{|c|c|}
\hline Frequency of Top Gate $(\omega)$ & $6 \times 10^{9} \mathrm{~s}^{-1}$ \\
Top Gate Capacitance $\left(C_{1}\right)$ & $9.07 \times 10^{-13} \mathrm{~F}$ \\
Back Gate Capacitance $\left(C_{2}\right)$ & $4.24 \times 10^{-13} \mathrm{~F}$ \\
Inductance $(L)$ & $7.76 \times 10^{-10} \mathrm{H}$ \\
Channel Resistance $(R)$ & $1.39 \times 10^{3} \Omega$ \\
\hline
\end{tabular}




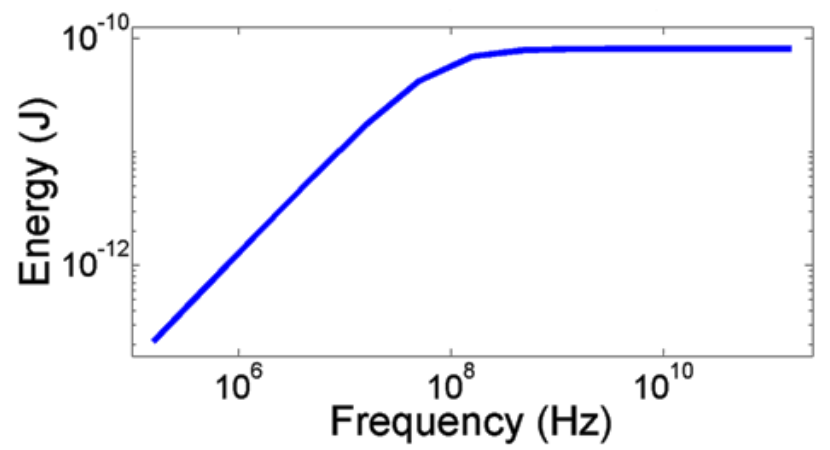

Figure 2.12: Energy dissipated as a function of $\omega_{L}$ for a fixed input energy on the inductor. The energy dissipated is calculated by taking the integral of the dissipated power $I^{2} R$ at $t=\infty$. The energy goes to almost zero in the adiabatic limit.

where $\omega_{L}=1 / \sqrt{L C_{1}}$. Next, we substitute (2.28) into (2.27) to derive

$$
\frac{d Q_{2}}{d t}+\frac{Q_{2}}{R C_{2}}=-\frac{I_{0}}{\omega_{L} R C_{1}} \sin \omega_{L} t
$$

Let $\omega_{1}=1 / R C_{1}$ and $\omega_{2}=1 / R C_{2}$. Therefore, equation (2.29) has the solution (with boundary condition $\left.Q_{2}(0)=0\right)$ :

$$
Q_{2}(t)=-\frac{I_{0} \omega_{1}}{\omega_{L}^{2}+\omega_{2}^{2}} \exp \left(-\omega_{2} t\right)-\frac{I_{0} \omega_{1}}{\omega_{L}\left(\omega_{L}^{2}+\omega_{2}^{2}\right)}\left(\omega_{2} \sin \omega_{L} t-\omega_{L} \cos \omega_{L} t\right)
$$

Therefore, the back gate charging current is

$$
I(t)=\frac{I_{0} \omega_{1} \omega_{2}}{\omega_{L}^{2}+\omega_{2}^{2}} \exp \left(-\omega_{2} t\right)-\frac{I_{0} \omega_{1}}{\left(\omega_{L}^{2}+\omega_{2}^{2}\right)}\left(\omega_{L} \sin \omega_{L} t+\omega_{2} \cos \omega_{L} t\right)
$$

(2.31) is only valid during times when $I(t) \geq 0$. This condition requires that

$$
\cos \omega_{L} t\left(\frac{\omega_{L}}{\omega_{2}}\right) \sin \omega_{L} t \leq \exp \left(-\omega_{2} t\right)
$$

2.32 suggests that there are periods when the back gate capacitor stops charging. During these periods, the charge is maintained on the back gate capacitor, as we will see later in the numerical simulations.

Let us now look at the adiabatic limit for the energy dissipation, by taking $\omega_{L} \rightarrow 0$. However, we must make sure that the initial energy on the inductor is unaffected by this limit. Since $\omega_{L}=1 / \sqrt{L C_{1}}$, L increases quadratically as we decrease $\omega_{L}$. Now, the initial energy on the inductor is given by 
$E_{\text {ind }}=\frac{1}{2} L I_{0}^{2} \propto\left(I_{0} / \omega_{L}\right)^{2}$. Therefore, we must decrease $\omega_{L}$ in such a way that $I_{0} / \omega_{L}$ remains constant. It is easy to see that the second and third terms in (2.31) go to zero as $\omega_{L} \rightarrow 0$. The first term also goes to zero if we re-write the equation as

$$
\lim _{\omega_{L} \rightarrow 0} I(t)=\frac{I_{0} \omega_{1} \omega_{2}}{\omega_{L}\left(\omega_{L}^{2}+\omega_{2}^{2}\right)} \omega_{L} \exp \left(-\omega_{2} t\right)=0
$$

With $I \rightarrow 0$, the dissipated energy through the resistor, $E_{\text {diss }}=I^{2} R$ also goes to zero. This implies that the total energy dissipated can be reduced by arbitrarily lowering the clock frequency, as predicted by the adiabatic limit (see Fig. 2.12).

\subsubsection{Ratchet-Based Gates}

The open-circuit voltage generated across a ratchet can be used to move the Fermi energy across another ratchet, i.e., to electrostatically "dope" it (see Fig. 2.13). Electronic ratchets can be doped to either n-type or p-type, as shown in the Fig. 2.14B, by charging the back gate capacitor. CMOS-like combinations of an n-ratchet and a p-ratchet can further create universal ratchet based logic gates such as INV, NAND, and NOR. In theory, electronic ratchets are capable of realizing any Boolean logic operation based on these three gates. For example, a ratchet inverter can be realized with an n-type and p-type ratchet, as shown in Fig. 2.14A. As seen in the figure, the magnitudes of $I_{A}$ and $I_{B}$ (currents from the p-type and n-type ratchets) can be manipulated through the back gate, which adjusts the Fermi level in the channel (see Fig. 2.14B). Furthermore, the output $V_{O C}$ is used to drive the back gate of the next ratchet in the circuit (shown as $V_{O C}$ in Fig. 2.14C). The sign of the current difference between $I_{A}$ and $I_{B}$ determines the charging or discharging of the next ratchet's

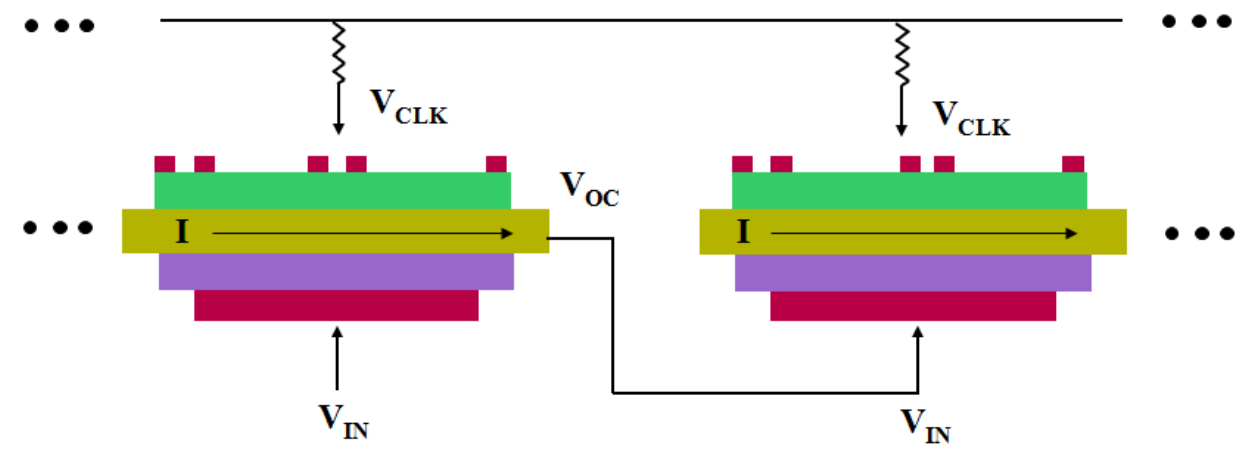

Figure 2.13: Logic circuits are built by cascading ratchet gates together. The first ratchet builds up an open-circuit voltage which is then used to charge or discharge the back gate of the next ratchet. 
(A)
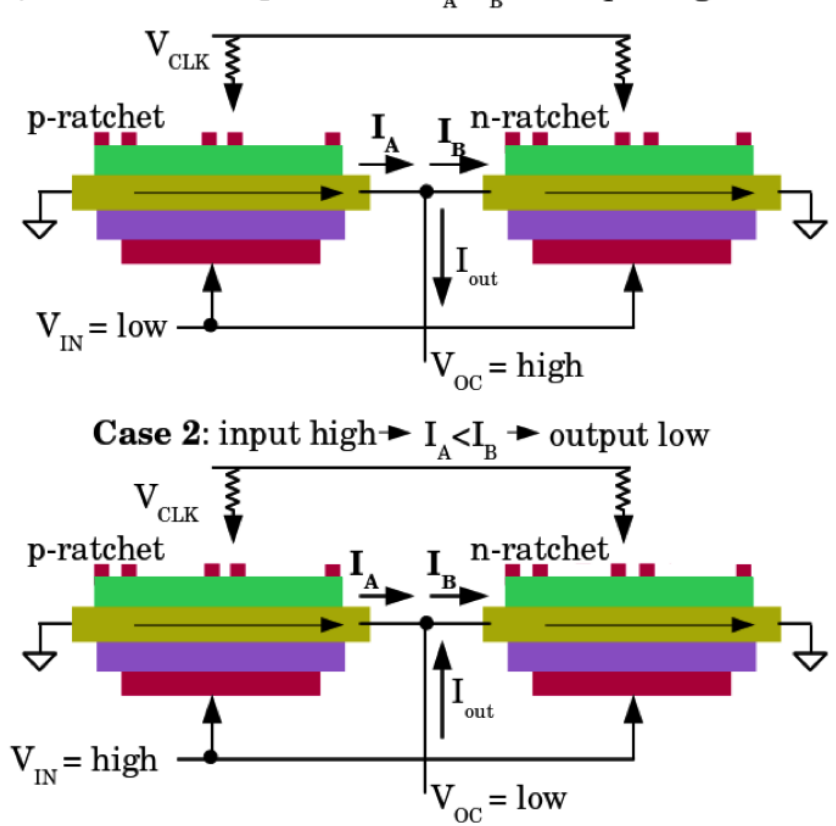

(B)
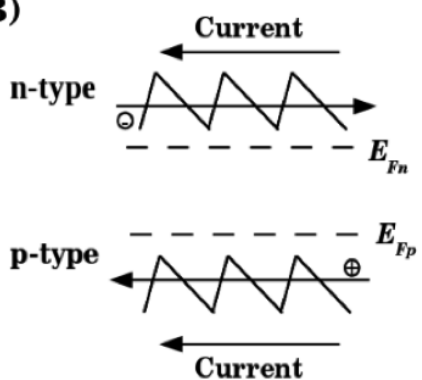

(C)

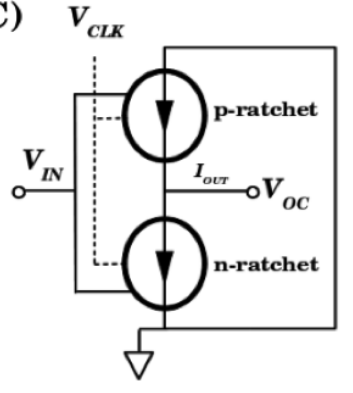

Figure 2.14: Ratchet inverter computing mechanism. (A, Case 1) $V_{\text {in }}$ is low and moves Fermi level down shown in (B), p-ratchet will have a higher current than n-ratchet so the output will charge the next capacitor $C_{b g}$ until the capacitor voltage reaches $-V_{O C}$ (low). $V_{i n}$ is high the output will discharge next capacitor $C_{b g}$ from $V_{O C}$ (high) to zero (A, Case 2).

back gate capacitor. When the input is high, the Fermi-level of the n-type devices shifts into the conduction band, allowing a large number of electrons to conduct and generate a high magnitude current $\left(I_{B}\right)$. In the p-type ratchet, high input bias shifts the $E_{F}$ away from the conduction band, thus lowering the current $\left(I_{A}\right)$. When $I_{B}$ is larger than $I_{A}$, the output of the inverter will start to discharge the next logic element's back gate capacitor.

In an analogous process, ratchet-based NAND gates and NOR gates can be created with two p-type and two n-type ratchets, with an extra balance capacitor between ratchets in pull-down network of NAND or pull-up network of NOR, to balance the current (as shown in the Fig. 2.15). The balance capacitor helps the model to have a stable transient current, so that the gates do not have meta-stable states between transitions.

In order to further capture the cascading behavior of the ratchet in the circuit model, we have modified the Schottky diode in the circuit to be a three terminal device, i.e., a voltage controlled rectifier. The third terminal is dependent on the open-circuit voltage built across the previous ratchet. 


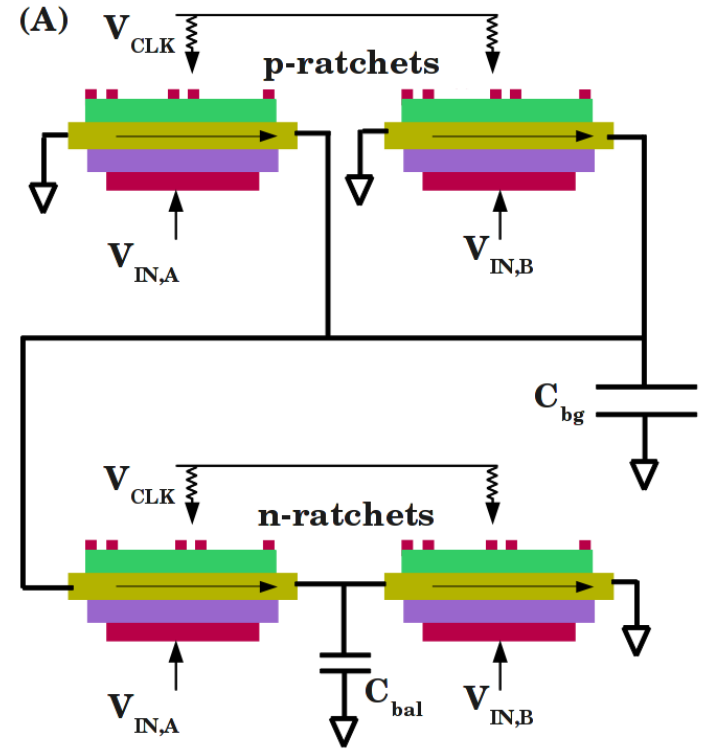

(B)

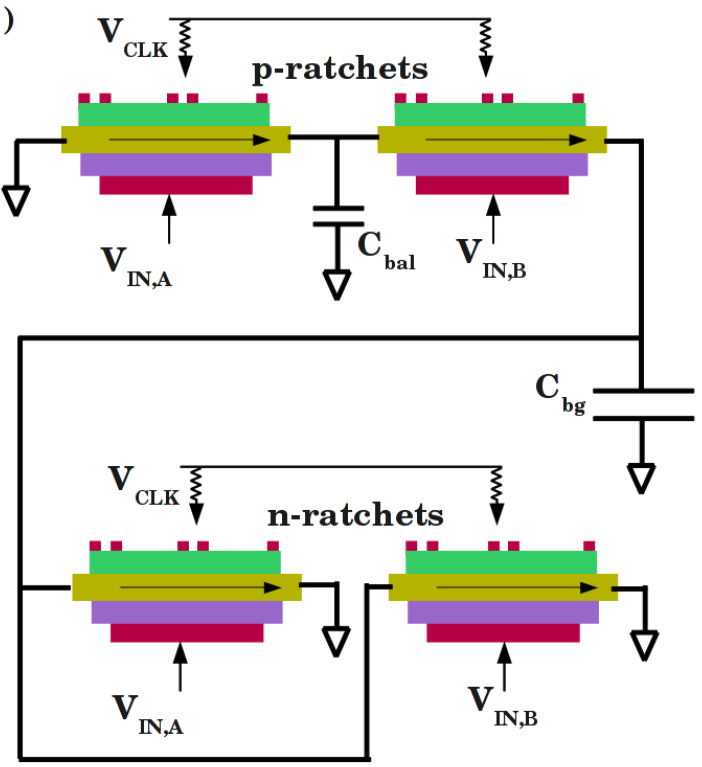

Figure 2.15: (A) Ratchet NAND gate and (B) NOR gate with the extra balance capacitors. Balance capacitors helps the models to have stable transient current, thus eliminating the metastability during the transitions.

This voltage adjusts the turn-on voltage for the device, thus mimicking the ON and OFF states of a ratchet based on the back gate voltage. In Fig. 2.13, we show how the voltage across the second ratchet diode is created by the open-circuit voltage from the first ratchet.

We simulated the circuit in Fig. 2.11 using Virtuoso Spectre. For the values of the circuit elements, we use the parameters listed on Table 2.2. In order to adjust the clock frequency, we changed the inductance and also the initial current so that the energy stored on the inductor remains constant through all clocking frequencies. We show the charging of the back gate capacitor to the open-circuit voltage in Fig. 2.16. As predicted from (2.32), the clock charges the back gate only during the times when the the current is positive (potential is up) and discharges when the current is negative (potential is down). We observe a qualitatively matching $V_{O C}$ built-up between the circuit simulations and the drift-diffusion simulations, although the circuit model can not capture the back-flow of particles when the potential is down. The $V_{O C}$ estimated is slightly higher than the calculated value given the simplicity of circuit model and that the ideal diode missing the back-flow shown in Fig. 2.7A.

Fig. 2.12 shows the energy dissipated in the resistor as a function of the top gate oscillation frequency. We can see that the energy continues to decrease as we scale down the oscillation frequency 


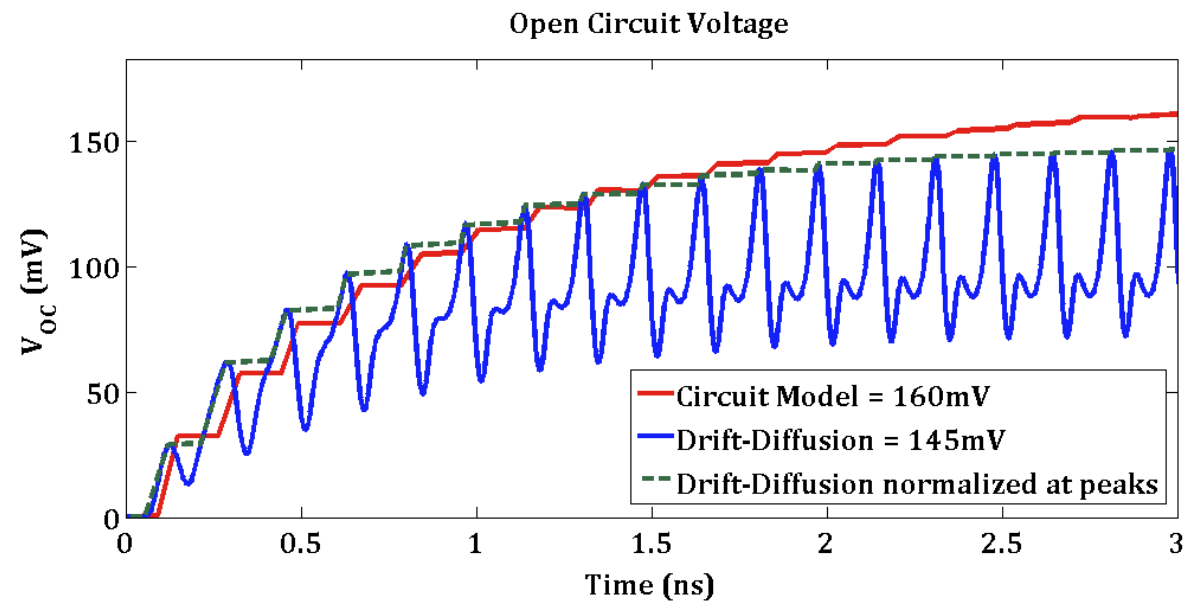

Figure 2.16: Circuit simulation using the circuit diagram in Fig. 2.11 showing the following results: The voltage being stored on the back gate capacitor compared with the drift-diffusion simulations. For the values of the circuit elements, we use the parameters listed on Table 2.2 and for the Schottky parameters and a barrier height of $15 \mathrm{kT}$ and $I_{0}$ of $1 \mathrm{E}-15 \mathrm{~A}$. Both of the results converge close to $\approx 145 \mathrm{mV}$ and $\approx 160 \mathrm{mV}$, slightly over shooting the expected result of $\approx 125 \mathrm{mV}$. The curves differ in that the circuit model does not capture the back-flow of the electrons as open-circuit builds-up, so it slightly over shoots the actual voltage built-up.

as predicted by the adiabatic limit. For high frequencies $\left(\omega_{L} \gg \omega_{2}\right)$, we begin to recover $(2.25)$ of the conventional charging mechanism.

\subsection{Limitations and Challenges}

This chapter demonstrates how, in principle, electronic ratchets can be used to perform logic operations at low energy costs, and how energy dissipation can be further reduced by the adiabatic charging process in the absence of timing constraints. However, there are a few critical effects that can still limit the overall performance of the electronic ratchet. When the electron drift and diffusion speed is comparable to that of the ions, innovative material and device engineering may be able to circumvent these limitations.

The energy dissipated by the ratchet can be arbitrarily reduced by turning down the clocking frequency. However, in order to have the ratchet operational, there must be a lower limit to the frequency. It is essential to note that the ratchet is ultimately a non-equilibrium device. If the electrons are allowed to diffuse continuously by lowering the asymmetric saw-tooth potential for too long, a near homogeneous distribution will be reached. When the barriers raise again, the drift current component becomes smaller. This property is presented in 2.10: as $t_{\text {off }}$ is increased, both error 
function terms become smaller and their difference decreases. Electrons move at high speeds through a small-channel, high-mobility material. This travel requires a minimum frequency that is fast enough to avoid the homogenization of electron distribution. In other words, a balance between the mobility of the channel material and the clocking frequency must be maintained in order to achieve optimal performance from the ratchet. The ion pumps in the neuronal networks do not encounter this problem of clocking because the ions have a much smaller mobility when moving through the ion pump.

As we scale ratchets towards nano-scale dimensions, we invariably reduce the distance between barriers. The clocking frequencies must be significantly increased in order to maintain the functionality of the ratchet at small dimensions. For a nanometer-sized device, the calculations and simulations predict that the frequencies must be in the Terahertz range in order to generate enough current to drive the next gate. This clock frequency problem can be bypassed by using a multi-phased clock to slow down the electrons. A peristaltic ratchet uses multiple clocks with different spatial and temporal phases to achieve directionality. In theory, this multi-clock system can slow the clock frequencies by a few orders of magnitude. Initially, the top gate clock localizes the electrons. When the barrier is lowered, electrons begin to diffuse through the channel. At this point, a second clock raises another set of barriers that are slightly offset spatially. These barriers begin to localize the electrons once again. Because of the spatial offset and temporal phases, the electrons reach a net directionality at lower clock frequencies.

In addition to the issue of compatibility between intrinsic and imposed frequencies, ratchets must overcome design limitation. While the creation of a clock-driven current source in the absence of a drain bias appears to be an advantage, transducing that current into a capacitive voltage (influenced by a design that is ultimately CMOS-centric) eventually neutralizes the current gain. As the ratchet system pumps electrons into the load capacitor, it builds up an open-circuit voltage over time. This accumulated voltage, however, acts as an effective drain bias that reintroduces the static dissipation into the logic circuit. To mitigate this issue, an alternative method of implementing logic circuits needs to be devised. Creating a current-based logic system, which would eschew the static dissipation, at least for several intermediate logic stages, may be possible. For now, we present this simple method as a demonstration of ratchet logic design. [33] shows that power efficient computation with a limited number of electrons can be achieved in ratchet-like geometry through the use of purely current driven Binary Decision Design (BDD) Circuits. 

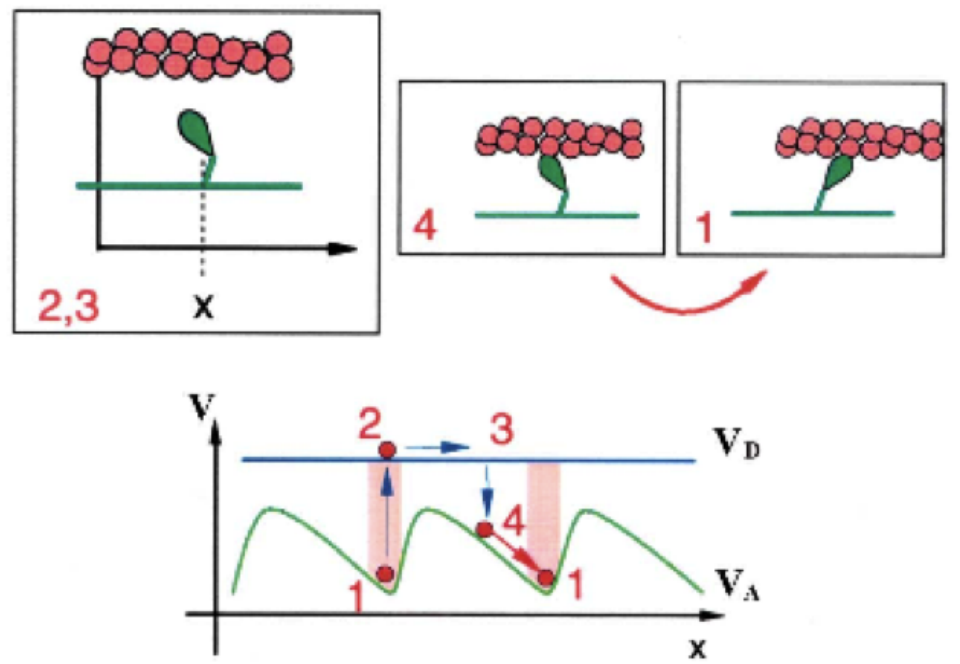
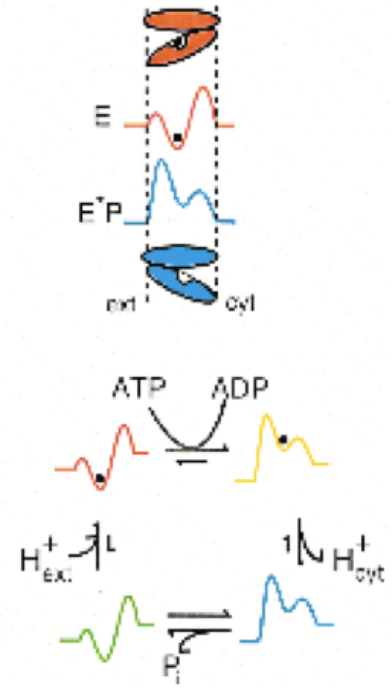

Figure 2.17: The ratchet model for creating an asymmetric potential (similar to the electronic ratchet simulations in previous sections) using the ATP hydrolysis to achieve a free Brownian motion [9]. The peristaltic illustration of a protein similar to the ion pumps with two conformational states: One with easy access from the right and one with easy access form the left. The switching between two conformations is induced by the ATP hydrolysis [9, 10].

\subsection{Conclusions and Contributions}

Biological systems present numerous examples of ratchet-like mechanisms that purport to shuttle elements (typically motor proteins) in the absence of global potentials. Ionic pumps powered by non-equilibrium signals such as the hydrolysis of ATP molecules, are excellent models of these biological elements. Ion pumps use a similar mechanism to the flashing ratchet to push the ions against their gradient. To create a flashing asymmetric potential profile, ion pumps utilize the attachment and detachment of dipoles. As this potential profile turns $\mathrm{ON}$ and $\mathrm{OFF}$, the ions are actively pushed from one direction to the other (see Fig. 2.17).

The contributions of this chapter are four-fold: (1) to extend the concept of biological ratchet-like mechanisms to a solid-state device, by using a clock and a series of potentials created by interdigitated electrodes; (2) to show how such a ratchet device can be cascaded to create universal Boolean logic; (3) to explain the possible energy-saving advantages arising from the reduced dynamic and static dissipation; and (4) finally, to outline limitations exposed by the quantum and classical simulations of our studied device geometries, namely, the importance of switching to current-driven (as opposed to voltage-driven) logic in the future, and the need to achieve a temporal matching or resonance between driving and driven frequencies. 


\section{Chapter 3}

\section{Nano-Electro-Mechanical Relays}

Digital electronics are fundamentally constrained by the cost of binary switching $Q \Delta V$. The number of charges $(N=Q / q)$ is relatively large (between 10-100 in the past 10 years) for a n-MOS transistor and ramps up rapidly to $\sim 1000 . \Delta V$ on the other hand is constrained by the Boltzmann-ShannonLandauer limit and corresponds to a switching slope of $k_{B} T \ln 10 / q \sim 60 \mathrm{mV} /$ decade. In order to perform switching efficiently, biological networks (such as axonal networks) utilize correlated and phase-transition switches to bypass these constraints. Voltage-gated ion channels in axons experience a phase transition to limit the energy cost by transferring ions along a concentration gradient [64]. Ion channels also operate near a phase transition point, so that a small voltage precipitates a large change [65]. The main goal of this chapter is to explain electron transport physics in a nano-mechanical relay switch, starting with the microscopic Landauer equation, which also elegantly explains the limits on the subthreshold swing. We develop the mathematical model and the physical picture behind the entire cantilever dynamics in its ON, OFF, and transitory states, the geometry of the hysteresis and its dependence on material parameters as well as the voltage scan rate. We argue that, similarly to the ion channels, a dipole-coupled, cantilever based relay can function as both a correlated switch and a phase transition switch. Therefore, the nano-mechanical relays can be used to improve our physical understanding of the ion channels' efficient switching properties (see Fig. 3.1).

This chapter presents a Landauer based microscopic transport model for electrons in cantilever based dipole-coupled nano-electro-mechanical field effect transistors. A thermal transition between a metastable state and a stable ground state in the energy landscape drives the dynamics. The energy landscape is composed of the elastic energy of the cantilever, the dipolar torque exerted by an 


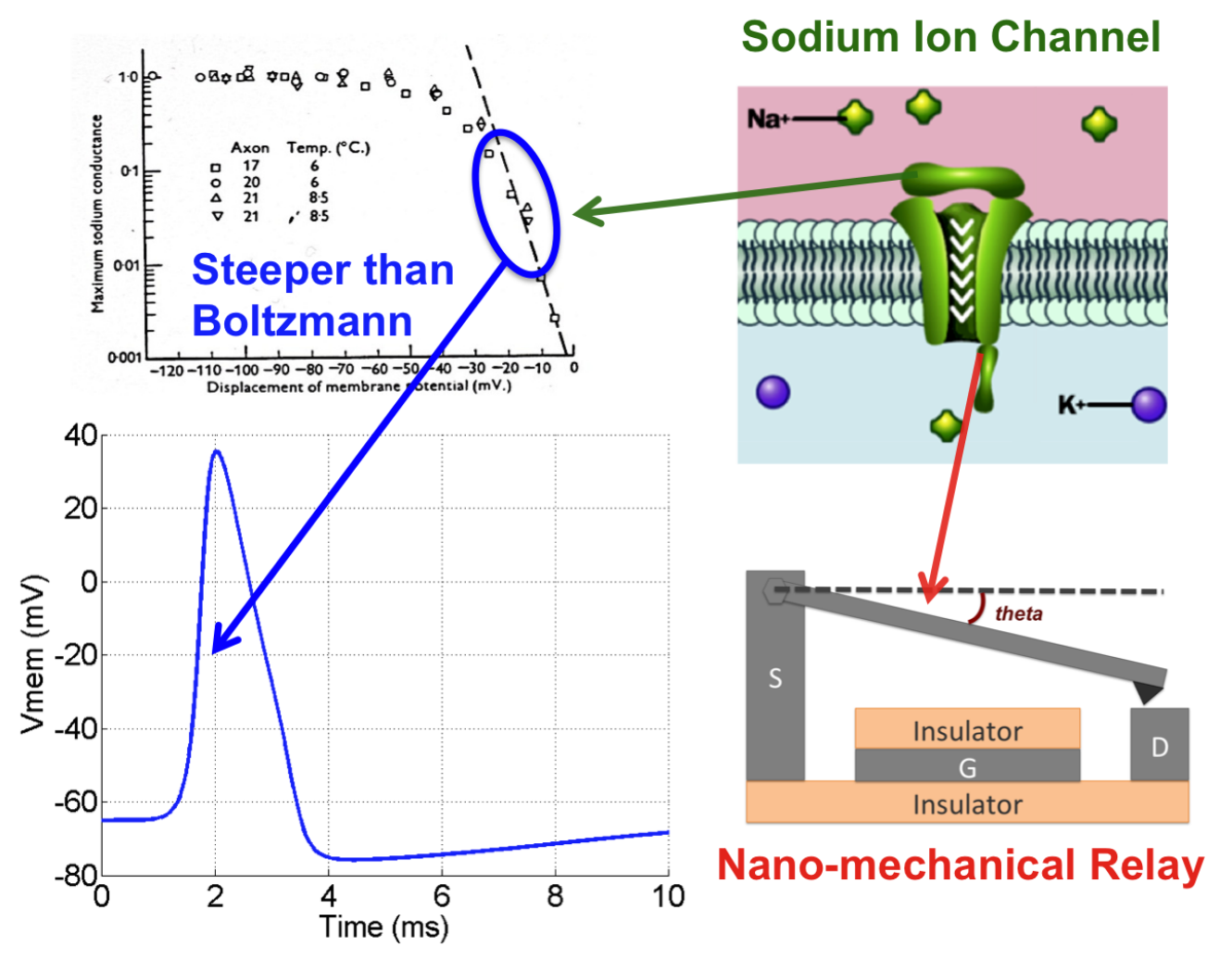

Figure 3.1: Ion channels operate in the rising and falling phases of the action potential to steeply increase and decrease the membrane voltage. Because ion channels and nano-electro-mechanical relays both have the same steep switching characteristics [3, 11], a connection can be made. In the ion channels, the cantilever shaped protein lid moves conformationally and opens the pores allowing the passive transport of ions for the expense of one (charge multiplication). In the nano-electro-mechanical FETs, the relay conformationally moves and touches the drain contact to generate a high current jump.

external gate, and a short-ranged pull-in force exerted by a van der Waals interaction at the drain. The individual pull-in and pull-out phases demonstrate a $6.5 \mathrm{mV} /$ decade subthreshold swing and are driven by both a dipole-based charge correlation effect and an abrupt phase transition that closes the air gap between the end of the cantilever and the drain. This sharp switching is similar to that seen in sodium ion channels. However, it comes at the expense of strong hysteresis as the metastable and stable states switch sides along the forward and reverse phases of the voltage scan.

\subsection{State of Practice for Nano-Electro-Mechanical FETs}

Current fabricated mechanical switches can swing between near zero OFF currents and high ON currents at very low $V_{D D}$ values [66]. Such nano-electro-mechanical field-effect-transistors (NEMFETs) are relevant as low power switches [67]. Ultra-low subthreshold swings have been demonstrated using 
suspended-gates [11] and micro-relays [67, 40]. For logic application, micro-relays demonstrated large voltage noise margins and excellent reliability [39]. Multiple designs, including the printable relay [68] and the curved relay by IBM [69], have been demonstrated.

The action of a NEMFET is significantly different from that of an electrostatically driven CMOS, and needs new physics-based predictive models. A number of empirical models have already been proposed based on $1 \mathrm{D}$ electrostatics $[40,70]$ and non-linear oscillator equations $[71,72,73]$. To improve understanding of the efficient switching characteristics, pull-in instability [74, 75] and deformation characteristics of microstructures under electrostatic loads [76] were modeled. Techniques from chaos and bifurcation theory in finite-elements were developed to analyze the phase-transition of these nano-mechanical relays $[77,78,79]$. However, none of these past models have the flexibility of our model introduced in this chapter. Our model captures the operations of both nano-mechanical relay devices (in micron sizes) and the smaller dimension biological and molecular components (in nanometer sizes). The model is able to incorporate both the dipole moment and the capacitive forces that bend the cantilever. Additionally, as the aspect sizes get smaller, our model captures the effects of adhesion forces.

\subsection{Device Structure}

The nano-electro-mechnanical switches exist in various flavors - movable gates or movable channels. Movable gate switches are driven by the capacitive energy $C V_{G}^{2} / 2$, where the capacitance

$C=A\left(t_{o x} / \epsilon_{o x}+t_{a i r} / \epsilon_{0}\right)^{-1}$ is divided serially between a fixed oxide and a variable air gap. Upon application of a gate bias, a force $F=-\partial U / \partial V_{G}$ alters the air gap from its equilibrium value $t_{0}$, counterbalanced by a spring, which exerts a restoring force $-k\left(t_{a i r}-t_{0}\right)[80]$. A short-ranged van der Waals force from a fixed oxide can attract the gate when it is close, and precipitate a sudden rise in capacitance. Since the pull-in acts on a capacitive structure, it is possible play with material properties of the gate oxide and shape its potential landscape in a creative way in order to liberate the movable gate easily. However, since the channel itself is fixed, its OFF current is solely limited by electrostatic depletion. For a small channel, it suffers from the usual source-to-drain tunneling, although the removal of the movable gate reduces the gate oxide leakage significantly.

In a movable channel transistor, the idea is to physically separate the channel away from the drain so that in addition to electrostatic depletion, the OFF current can be reduced substantially 

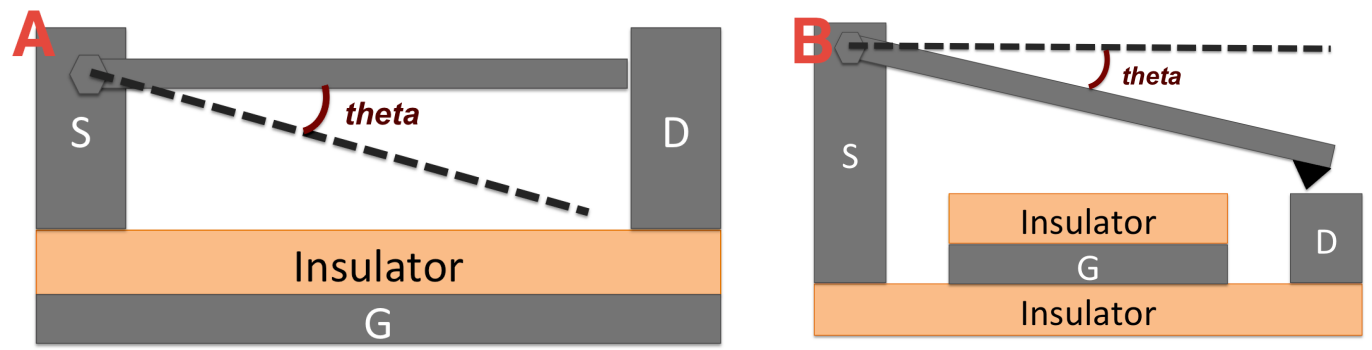

Figure 3.2: (A) In normally ON device structure, the cantilever starts with contacting both the drain and source electrodes, resulting in active current flow. When a positive gate bias is applied, it moves the cantilever down, increasing the angle "theta". When the cantilever looses it's contact to the drain, the current flow suddenly stops, resulting in the OFF configuration. To turn the device back ON a large reverse bias on the gate is applied until the cantilever makes contact with drain again. (B) Normally OFF device structure where the cantilever starts away from drain. As positive bias on the gate pulls lever down, increasing the angle "theta". Once the cantilever contacts to drain, the current jumps, tuning the device ON. The cantilever is released back to dashed line to the OFF configuration when a large reverse bias is applied on the gate.

with an added quantum mechanical tunneling barrier (see Fig. 3.2). In fact, it is straightforward to show that the electrostatic and the conformational modes and thus their subthreshold swings act in parallel, $S^{-1} \approx S_{e l}^{-1}+S_{\text {conf }}^{-1}[37]$, assuming a clear separation of electronic and conformational time constants. Furthermore, the conformational mode, besides aiding the electrostatic turn OFF, allows an intrinsically low subthreshold swing

$$
S_{\text {conf }}=\frac{k_{B} T}{q} \ln 10\left[\frac{q\left(t_{o x}+t_{\text {air }}\right)}{\mu}\right] C(\theta)
$$

where $C$ is a correlation function that we will describe later the chapter. The cantilever FET can function as both a correlated switch and a phase transition switch. The presence of the dipole $\mu$ means that the subthreshold swing can be reduced by a factor $q t_{o x} / \mu \propto t_{o x} / L$ for longer cantilevers, exploiting the property that many charges are physically moving with the cantilever for the price of one (charge multiplication). This is, in fact, the suggested mechanism behind the observed sharp switching in voltage-gated sodium channels [64]. A separate mechanism arises from an additional "pull-in" that grabs the end of the cantilever once it is physically proximal with the drain. The short-ranged van der Waals force causes an abrupt shrinkage of the air gap and an exponential rise in tunneling current, reducing the subthreshold swing further by a factor proportional to $d t_{a i r} / d \theta$, which vanishes near the contact point, giving a near vanishing subthreshold swing. The van der Waals pull-in helps subvert thermal fluctuations by stabilizing the attractive forces. Resetting the device however, requires circumventing adhesion, which creates a sizable hysteresis loop. The switch 
thus has extremely sharp transitions along the walls of the hysteresis curve, but its loop-averaged subthreshold swing is quite large and problematic for high performance electronic applications.

\subsection{Transport Equations}

The electron current is determined by the degree of quantum mechanical tunneling across the gap between the cantilever and the drain. We can describe that using the Landauer equation [81], written in an equivalent, but less familiar form as a convolution between the zero-temperature current and a thermal broadening function $F_{T}(E)=-\partial f(E) / \partial E$

$$
I=\frac{2 q}{h} \int_{\mu_{1}}^{\mu_{2}} d E M(E)\left\langle\bar{T}\left(E, V_{G}\right)\right\rangle \otimes F_{T}\left(E, V_{G}\right)
$$

where $M(E)$ is the mode count in the cantilever, $f$ is the equilibrium Fermi-Dirac distribution, with the $V_{G}$ dependence in $F_{T}$ arising from the location of the Fermi energy $E_{F}$, which shifts with $V_{G}$ scaled by a gate transfer factor $\alpha_{G} \cdot \mu_{1,2}$ are the bias separated electrochemical potentials in the source and drain contacts, and $\bar{T}\left(E, V_{G}\right)$ is the mode and conformation averaged transmission function controlled by the gate voltage-dependent orientation. 〈...〉 represents the thermodynamic average of the cantilever orientation $\theta$ over various angles, weighted by its probability distribution $P$

$$
\left\langle\bar{T}\left(E, V_{G}\right)\right\rangle=\frac{\int d \theta \bar{T}(E, \theta) P\left(\theta, V_{G}\right)}{\int d \theta P\left(\theta, V_{G}\right)}
$$

If we assume the mechanical modes in the cantilever are at equilibrium, (i.e., the potential is ramped in time much slower than the relaxation scales in the lattice), $P$ is simply the Boltzmann solution $\propto \exp \left[-U\left(\theta, V_{G}\right) / k_{B} T\right]$, even though the electrons flowing are clearly in non-equilibrium. In general, the cantilever's angular distribution satisfies a thermodynamic equation that needs to be derived properly from a stochastic Langevin equation in the presence of a fast ramp speed of the potential. A flexible cantilever clamped on one end and free on the other satisfies a fourth degree partial differential equation of motion. For an over-damped, rigid cantilever with a single defining angle

$\theta$, the equation can be simplified down to the second degree and consequently $P$ can be shown to satisfy the Fokker-Planck equation

$$
\gamma \frac{\partial P}{\partial t}=\frac{\partial}{\partial \theta}\left(P \frac{\partial U\left(\theta, V_{G}\right)}{\partial \theta}\right)+k_{B} T \frac{\partial^{2} P}{\partial \theta^{2}}
$$


where $\gamma$ is the angular damping constant with units J-s. We assume here that all phonons emitted in the cantilever are promptly reabsorbed by a conductive thermal coating coupled to the source, so we can ignore any self-heating and phonon bottleneck effects in the cantilever itself.

The above equations carry all the necessary ingredients for our NEMFET operation. The normal subthreshold swing of $k_{B} T \ln 10 / \alpha_{G} q$ arises from the gate dependent shift in mode count $M$ for a fixed orientation, when $U$ is a delta function around $\theta=0$, and $P$ is simply equilibrium Boltzmann. Further reduction in subthreshold swing will arise from the added gate dependence of the conformational modes, hidden in $U(\theta)$. The hysteresis will arise from a transition out of metastable states with a non-equilibrium distribution $P$ in presence of a time-dependent sweep in gate voltage, $V_{G}(t)$. Finally, a negative capacitance for a ferroelectric oxide will be captured by the gate voltage dependence of the oxide dielectric constant, $\epsilon_{o x}\left(V_{G}\right)$, which can introduce its own dynamics near the ferroelectric phase transition point.

\subsection{Operation of the Relay}

The physics of the relay is captured by nano-mechanical transmission and conformational potential profile. The transmission is limited by electron tunneling into the drain from the edge of the cantilever through the vacuum barrier of width $t_{\text {air }}(\theta)=h-L \sin \theta, h$ being the bare height above the drain before bending and $L$ being the length of the cantilever. The transmission, using a WKB approximation, is $\bar{T}(E, \theta) \propto \exp \left[-2 \kappa t_{a i r}(\theta)\right]$. The main physics of the NEMFET dynamics is captured by its potential $U\left(\theta, V_{G}\right)$, which can be separated into a flexural potential $U_{0}$ representing the tendency of the cantilever to snap back to its horizontal orientation, a gate driven field $\overrightarrow{\mathcal{E}}$, which couples with the dipole moment $\vec{\mu}$ in combination with the capacitive forces between the cantilever and the back gate, and finally a van der Waals "pull-in" potential $U_{V d w}$ that causes the cantilever to "stick" to the drain when close enough.

$$
\begin{aligned}
U\left[\theta, V_{G}\right] & =U_{0}(\theta)+U_{V d W}(\theta) \\
& +U_{d i p}\left[\theta, V_{G}\right]+U_{c a p}\left[\theta, V_{G}\right] \\
U_{0}(\theta) & \approx \frac{1}{2} U_{0}^{B e n d} \theta^{2} \\
U_{V d W}(\theta) & =4 \epsilon\left[\left(\frac{\sigma}{t_{\text {air }}(\theta)}\right)^{12}-\left(\frac{\sigma}{t_{\text {air }}(\theta)}\right)^{6}\right]
\end{aligned}
$$




$$
\begin{aligned}
& U_{d i p}\left[\theta, V_{G}\right]=-\vec{\mu} \cdot \overrightarrow{\mathcal{E}}=-\mu\left(\frac{V_{G} \sin \theta}{t_{o x} / \epsilon_{o x}+t_{a i r}(\theta) / \epsilon_{\text {air }}}\right) \\
& U_{c a p}\left[\theta, V_{G}\right]=-\frac{1}{2}\left(\frac{A}{t_{o x} / \epsilon_{o x}+t_{\text {air }}(\theta) / \epsilon_{\text {air }}}\right) V_{G}^{2}
\end{aligned}
$$

We assume a simple parabolic potential for the bending dynamics to focus on the basic physics, although higher nonlinear terms would be important to capture the full import of the flexural modes [82]. However, these more accurate analytical models for the cantilever shape do not provide additional insights [83]. Note also that a simple pull-in arises just from the capacitive forces between the cantilever and the back gate, captured by the energy, which we ignore in the face of the larger force applied on the dipole by the gate in the smaller device sizes. It is worth emphasizing that the dipolar torque has a distinct advantage over capacitive forces, as it exploits the directionality of the gate field. Even if the oxide is thick enough to normally produce short-channel effects, the gate field is orthogonal to the drain field and thus creates a larger torque than the latter (especially in the presence of dual gates), which makes its effect more pronounced. As the device sizes scale larger in to the micron regime, the width (W) becomes larger to stop these devices to start in the sticking position, so the effect of the capacitive forces starts to overcome the effect of the dipole forces.

Fig. 3.3A shows the flexural parabolic potential, progressively shifted by the external electric field, causing a change in average angular orientation. Fig. 3.3B shows the separate van der Waals potential that acts as a pull-in when the relay comes close enough to the drain. In combination of these two effects (see Fig. 3.3C), there is a sweet spot where the potential profile between the metastable oscillator minimum and the stable van der Waals minimum reaches a point of inflection, whereupon the cantilever angular coordinate makes an abrupt phase transition and locks onto the drain (in actuality this happens a bit earlier, once the hill separating the van der Waals and parabolic minima reduces to around $\sim k_{B} T$ ). The pull-in has an important role to play. In its absence, the

Table 3.1: Simulation Parameters for Model
\begin{tabular}{|c|c|c|c|}
\hline$L$ & $5 \mathrm{~nm}$ & $k_{B} T$ & $0.025 \mathrm{eV}$ \\
$h$ & $2.5 \mathrm{~nm}$ & $U_{0}^{\text {Bend }}$ & 2 \\
$W$ & $0.5 \mathrm{~nm}$ & $\vec{\mu}$ & $0.15 \mathrm{e} \AA$ \\
$t_{\text {air }}$ & $1.5 \mathrm{~nm}$ & $\epsilon_{\text {Relay }}$ & $3.98 \mathrm{eV}$ \\
$\epsilon_{\text {air }}$ & 1 & $\epsilon_{\text {MetalPlate }}$ & $0.67 \mathrm{eV}$ \\
$t_{o x}$ & $1 \mathrm{~nm}$ & $\sigma_{\text {Relay }}$ & $3.47 \AA$ \\
$\epsilon_{o x}$ & 3.9 & $\sigma_{\text {MetalPlate }}$ & $3.144 \AA$ \\
\hline
\end{tabular}



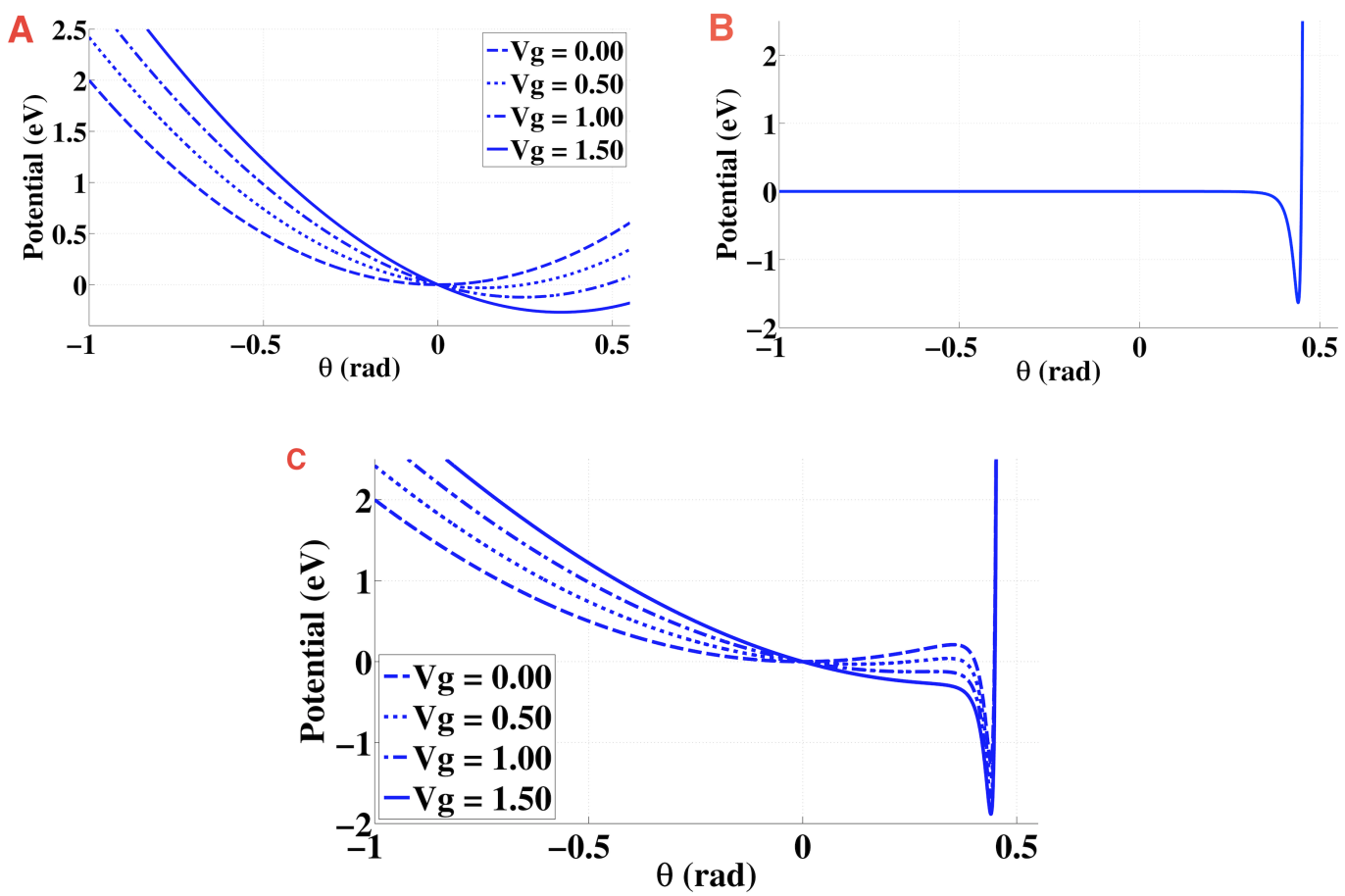

Figure 3.3: (A) The parabolic potential of the cantilever with one local minimum resulting from the combination of the stiffness and the external field terms. The local minimum shift with the gate bias. (B) The VDW forces resulting from the gate-electrode with a stationary local minimum. (C) A microscopic view of the parabolic potential varying with Gate bias in addition of a localized VDW forces from the contact equals in the NEMFET potential profile, which has one local minimum varying with the gate bias and a second localized minimum (see Table 3.1 for simulation parameters).

cantilever would be either too rigid to displace with an external field, or too floppy to resist thermal averages. In the absence of any other barrier, the thermal average over the conformational potential would tend to restore the system to its initial OFF state. This is a well known problem with a single well, analog computing - i.e., while it is energetically very pliable, it is highly corruptible with noise. The role of the van der Waals pull-in is to allow an initial low energy analog variation in coordinate, but then to insert a pseudo-digital component at a later stage of the game, once the system is ready to settle down near its ON state. In other words, it provides only a weak barrier to forward motion from the metastable to a stable state but a strong barrier for a return to origin.

This barrier asymmetry is at the heart of the NEMFET operation, but relies on the intrinsic non-equilibrium nature of the dynamics. Left to itself long enough, the cantilever would always find a way to thermally jump out from its metastable state to the stable state, i.e., it would bend over and stick to the drain given adequate time even in absence of a gate field assisting it. To define the transition between states, we will need to operate the voltage scanning faster than this spontaneous 

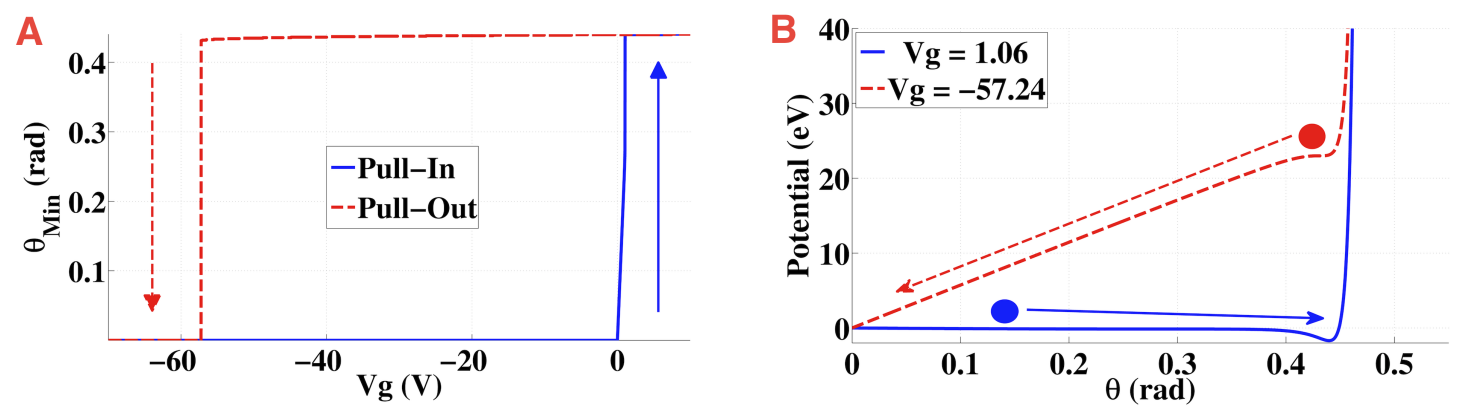

Figure 3.4: (A) Pull-in and pull-out mechanism rising from the NEMFET potential. The pullin occurs when the positive gate-bias lowers the local maximum (barrier) between the two local minimums allowing the particles to move right (shown by the red curves). (B) The negative voltage shifts the local minimum from the VDW forces to higher energies, thus allowing the particles to shift left to the local minimum from the parabola.

transition rate, so that the cantilever coordinate stays pinned to the metastable state until the voltage eliminates the barrier to the stable ground state.

Fig. 3.4 shows the pull-in and pull-out dynamics mechanism arising out of the gate dependent alteration of the two well potential. The pull-in occurs when a sufficiently high positive gate bias is applied between the gate and the source electrode until at a critical voltage $V_{P I}$, the local maximum between the two minima disappears. The pull-out requires a high negative gate voltage $V_{P O}$ in order to break the van der Waals adhesive bond. Described in terms of the potential landscape, the higher order flexural terms elevate the van der Waals minimum until the latter becomes the metastable state and the cantilever configuration makes a reverse phase transition back to the horizontal coordinate. The resulting potential and the average angle are shown in that figure. Clearly the $V_{P O}$ depends on the details of the van der Waals potential and the higher order terms in the flexural modes, and can be reduced by adjusting the contact geometry, material and surface properties such as its roughness and quality.

\subsection{Sharp Switching and Breaking the Landauer Limit}

Let us try to work out the phase transition physics and the associated reduction in subthreshold swing. For a long stiff cantilever, the fluctuations about the mean can be ignored, so that $P(\theta) \propto e^{-\beta \theta}$ becomes a delta function around the mean angle $\theta^{*}$ set by the gate voltage $V_{G}$. We can write the $U$ around its mean (where $U$ reaches an extrema) as $U(\theta) \approx U\left(\theta^{*}\right)+U^{\prime \prime}(\Delta \theta)^{2} / 2$, so that the 
fluctuations in the Boltzmann distribution look similar to a Gaussian

$$
e^{-\beta U(\theta)} \approx e^{-\beta U\left(\theta^{*}\right)} e^{-\beta U^{\prime \prime}(\Delta \theta)^{2} / 2}
$$

with standard deviation $1 / \sqrt{\beta U^{\prime \prime}}$. For a stiff cantilever compared to thermal fluctuations, we can approximate this Gaussian as a delta function around $\Delta \theta=0$, which then integrated over angle gives us

$$
I=\frac{2 q}{h} \int_{\mu_{1}}^{\mu_{2}} d E M(E) \bar{T}\left(E, \theta^{*}\left(V_{G}\right)\right) \otimes F_{T}\left(E, V_{G}\right)
$$

evaluated at a single gate-dependent angle $\theta^{*}\left(V_{G}\right)$. Differentiating with the gate voltage, we derive

$$
\frac{\partial I}{\partial V_{G}}=\frac{2 q}{h} \int_{\mu_{1}}^{\mu_{2}} d E M(E)\left[\frac{\partial \bar{T}}{\partial V_{G}} \otimes F_{T}+\bar{T} \otimes \frac{\partial F_{T}}{\partial V_{G}}\right]
$$

The tail of the Fermi-Dirac distribution makes $\partial F_{T} / \partial V_{G} \approx-\alpha_{G} \beta=-\alpha_{G} / k_{B} T$, while the derivative of the first term in the bracket can be written as $\left(\partial \bar{T} / \partial \theta^{*}\right)\left(\partial \theta^{*} / \partial V_{G}\right)$. The WKB tunneling term $\bar{T}=T_{0} \exp \left[-2 \kappa t_{\text {air }}\left(\theta^{*}\right)\right]$ gives $\partial \bar{T} / \partial \theta^{*}=-2 \kappa \bar{T} d t_{a i r}\left(\theta^{*}\right) / d \theta^{*}$. From geometry, $t_{a i r}\left(\theta^{*}\right)=$ $h-L \sin \theta^{*} \approx L\left(\theta_{0}-\theta^{*}\right)$ for a long cantilever where $0<\theta^{*}<\theta_{0} \approx h / L \ll 1$. Then $d t_{\text {air }}\left(\theta^{*}\right) / d \theta^{*} \approx-L$, and we get the normalized transconductance

$$
\bar{g}_{m}=-\frac{\partial \ln I}{\partial V_{G}}=\beta+2 \kappa L\left(\frac{d \theta^{*}}{d V_{G}}\right)
$$

for perfect gate control. The normalized transconductance is inversely proportional to the subthreshold swing to within a factor of $\ln 10 / q$. It is easy to see that the transconductance is already larger (and thus the subthreshold swing smaller) than the purely electrostatic limit $\alpha_{G} \beta$, and that this
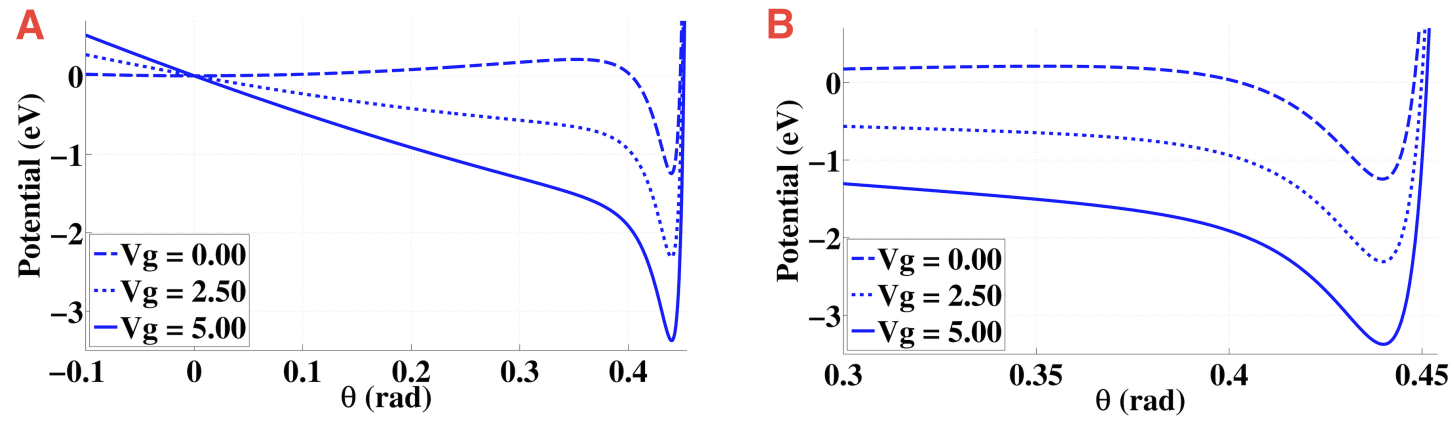

Figure 3.5: The movement of the minimum, $\theta^{*}$, is shown in the potential profile. The $V_{G}$ shifts the metastable local minimum of the first well, until the local minimum disappears and jumps into the global minimum of the van der Walls well. 


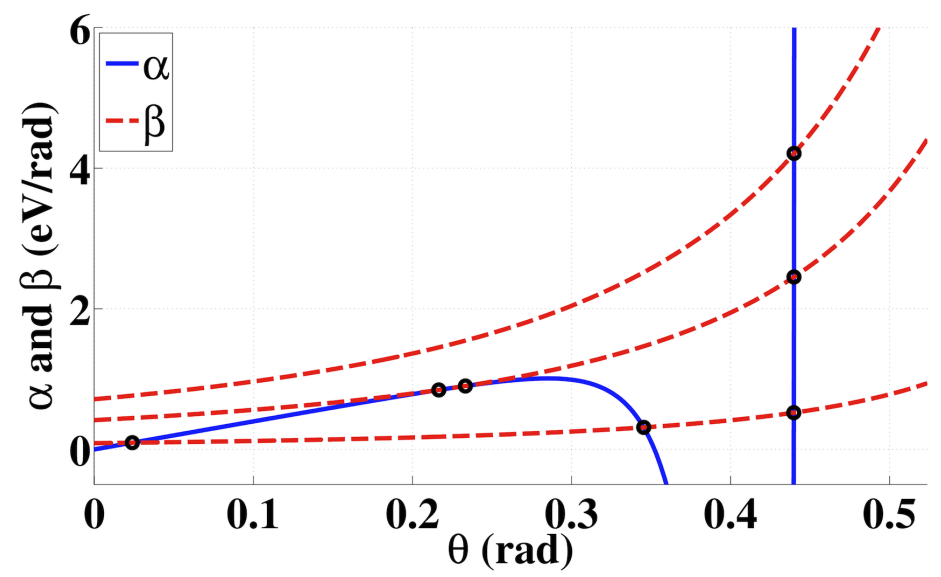

Figure 3.6: The transcendental equation obtained by taking $d U\left(\theta^{*}\right) / d \theta^{*}=0$ is shown as the intersection of set of two curves. First set of curves (dashed red) are shifting vertically up as the $V_{G}$ value is increased. For the low values of the $V_{G}$, there are three intersection points corresponding to the three extrema: the local minimum from cantilever, the global minimum from van der Waals, and the local maximum between two wells. At a the high enough $V_{G}$, local minimum and the local maximum collapses resulting with only the global minimum. This movement of the minimums is the main reason of the phase transition in the relays.

increase can diverge if either the cantilever is very long or if cantilever can be made to collapse abruptly with gate voltage $V_{G}$ so that $d \theta^{*} / d V_{G} \gg 1$.

The physics of the evolution of $\theta^{*}$ is easily seen from the potential profile in Fig. 3.5. The gate shifts the minimum of the first well (the metastable state) in an analog format, till the cantilever coordinate jumps into the van der Waals well. The minimum can be obtained by taking $d U\left(\theta^{*}\right) / d \theta^{*}=0$. This gives us a transcendental equation graphically shown in Fig. 3.6 as the intersection of two sets of curves, one of which shifts vertically with increasing $V_{G}$. For low values of $V_{G}$, you have three intersection points corresponding to three extrema - the two well bottoms and the bump in between. At high enough $V_{G}$ however, the wells merge and there is only one point of intersection to the right, the global minimum at the bottom of the van der Waals well, and this is what drives the phase transition.

We can simplify the algebra considerably by replacing the van der Waals potential with a stiff parabola, and ignoring the oxide thickness. We can then write

$$
\begin{aligned}
U(\theta) & \approx \frac{1}{2} U_{0}^{B e n d} \theta^{2}+\frac{1}{2} k_{V d W}\left(\theta-\theta_{0}\right)^{2} \\
& -\frac{\mu \epsilon_{a i r} V_{G} \theta}{L\left(\theta_{0}-\theta\right)}-\frac{A \epsilon_{a i r} V_{G}^{2}}{2 L\left(\theta_{0}-\theta\right)}
\end{aligned}
$$


Setting its derivative equal to zero to extract the potential minima, we get

$$
\begin{aligned}
0 & =U_{0}^{B e n d} \theta^{*}-k_{V d W}\left(\theta_{0}-\theta^{*}\right) \\
& -\frac{\mu \epsilon_{a i r} V_{G} \theta_{0}}{L\left(\theta_{0}-\theta^{*}\right)^{2}}-\frac{A \epsilon_{a i r} V_{G}^{2}}{2 L\left(\theta_{0}-\theta^{*}\right)^{2}}
\end{aligned}
$$

From the above equation, we can now extract the slope of the $\theta^{*}\left(V_{G}\right)$ curve, which enters the normalized transconductance $\bar{g}_{m}$

$$
\frac{d \theta^{*}}{d V_{G}}=\frac{\mu \epsilon_{a i r} \theta_{0} / L+A \epsilon_{a i r} V_{G} / L}{\left(3 k_{V d W}+U_{0}^{B e n d}\right)\left(\theta_{0}-\theta^{*}\right)^{2}+2 U_{0}^{B e n d} \theta^{*}\left(\theta_{0}-\theta^{*}\right)}
$$

It is easy to see that near the touching point, $\theta^{*} \approx \theta_{0}$, whereupon we can drop the first term in the denominator, and get

$$
\bar{g}_{m}=\beta+\frac{\kappa \epsilon_{a i r}}{U_{0}^{B e n d}}\left[\frac{\mu}{\left(\theta_{0}-\theta^{*}\right)}+\frac{A V_{G}}{\theta^{*}\left(\theta_{0}-\theta^{*}\right)}\right]
$$

As explained earlier, we can make the transconductance really high by either ramping up the total dipole moment $\mu$ or increasing the area with the applied bias or by collapsing the cantilever, $\theta^{*} \approx \theta_{0}$.

In practice, there is usually an oxide, with an inverse capacitance proportional to $t_{o x} / \epsilon_{o x}$ added to the inverse capacitance of the air gap, $t_{a i r} / \epsilon_{a i r}=L\left(\theta_{0}-\theta^{*}\right) / \epsilon_{\text {air }}$. We drop the first term, but when the inverse capacitance of the air collapses, we need to replace $\epsilon_{a i r} / L\left(\theta_{0}-\theta^{*}\right) \rightarrow \epsilon_{o x} / t_{o x}$, so that

$$
\left.\bar{g}_{m}\right|_{\text {Max }}=\beta+\frac{\kappa L \epsilon_{o x}}{U_{0}^{B e n d} t_{o x}}\left[\frac{\mu}{1}+\frac{A V_{G}}{\theta^{*}}\right]
$$

It is interesting that the strength of the van der Waals potential does not matter here, because we assume it is much stiffer than the cantilever, which then determines the rate limiting step. We need to make the cantilever very pliable $\left(U_{0}^{B e n d}\right.$ small), however, remember that we started the algebra by assuming weak thermal fluctuations, which require $\beta U^{\prime \prime}=\beta U_{0}^{B e n d} \gg 1$, in other words, the cantilever must be stiff with respect to thermal fluctuations but floppy relative to the van der Waals. The assumption $U_{0}^{B e n d} \beta \gg 1$ would still allow us to rely on the second term in 3.14 above, provided $\kappa L \epsilon_{o x} \mu / t_{o x} \gg U_{0}^{B e n d} \beta$. We therefore need a high tunneling decay length i.e., low tunneling effective mass, a long cantilever, a high dipole and a thin oxide. Needless to say, we also need good gate control, $\alpha_{G} \rightarrow 1$ (once reintroduced, it sits in front of the entire expression for $\bar{g}_{m}$ ). 

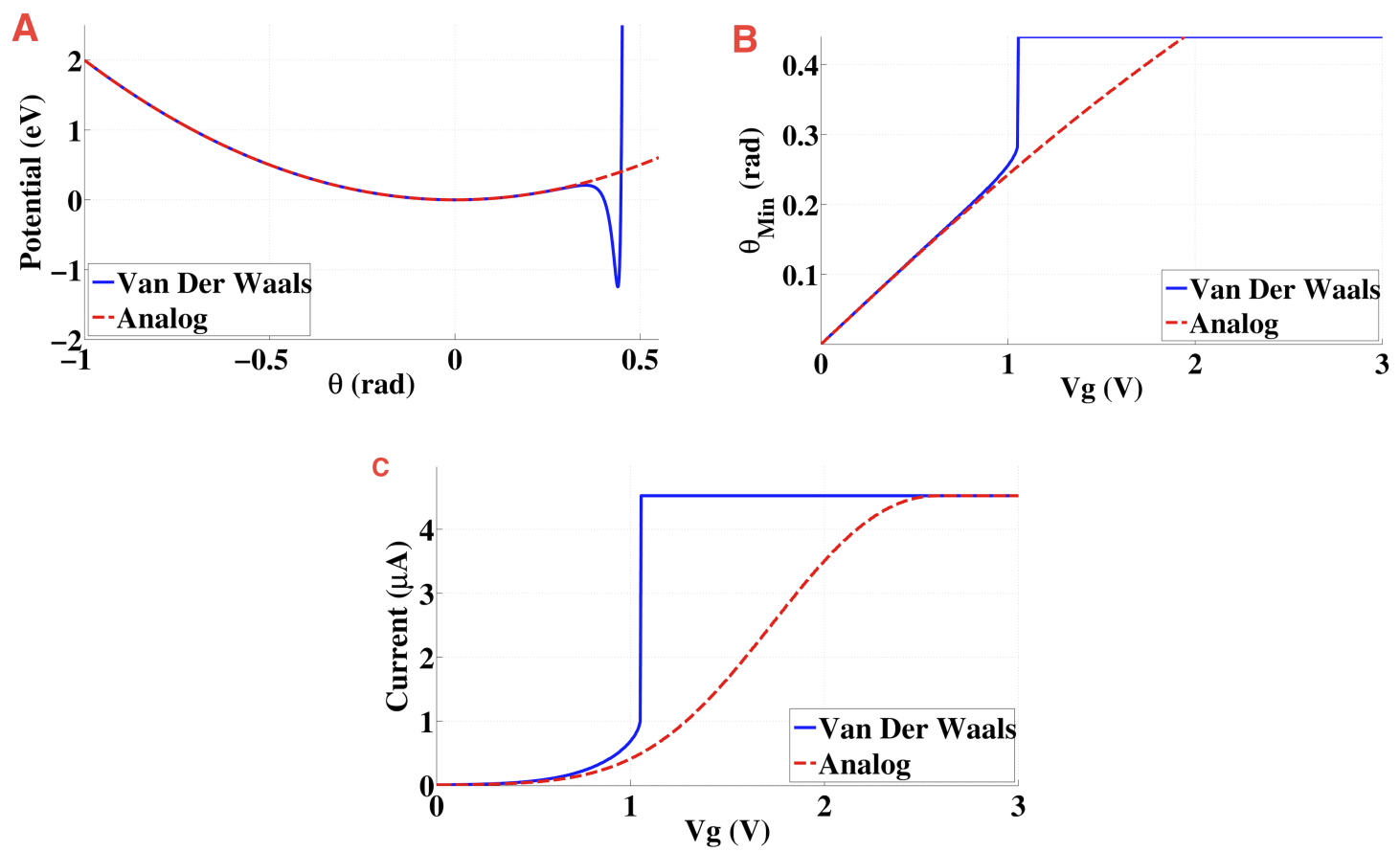

Figure 3.7: Comparison of the subthreshold swing with and without the addition of VDW forces. With the VDW forces the cantilever is pulled down instantaneously shown in red, causing an improved $\mathrm{SS}$ of $12 \mathrm{mV} / \mathrm{dev}$. Without the addition of VDW forces from the gate, the fastest the voltage swing is limited to thermal limit of $60 \mathrm{mV} / \mathrm{dev}$ as shown in dashed black line. Thus, proving the importance of the VDW forces at the nano-scale limit on the subthreshold swing.

In Fig. 3.7, we compare our physical model with and without the addition of the VDW forces. In the absence of the VDW forces, the performance is limited to $60 \mathrm{mV} /$ decade and the cantilever angle "theta" increases slowly, but with the VDW forces, the movement of the cantilever speeds up significantly with the addition of the second minimum in the potential, lowering the SS to $12 \mathrm{mV} / \mathrm{dev}$ (see Fig. 3.7C). Once the gate voltage increases enough to remove the local maximum shown in Fig. 3.3, the switching occurs by the tip of the cantilever making contact with the drain electrode.

\subsection{Elasticity versus Dipole and Capacitive Forces}

The cantilevers' elasticity compared to the dipole moment and the electric field, alters as the size of the cantilever changes. In the larger cantilevers, the electric field has much more dominant effect since the gate to the surface area of the cantilever $(A)$ is larger thus creating a very large capacitive force compared to the dipole moment force. Whereas in the smaller cantilevers similar to the molecular or the sodium/potassium ion gates, the dipole moment is much more dominant since the capacitive area is extremely small, thus the capacitance is insignificant compared to the high dipole moment. 


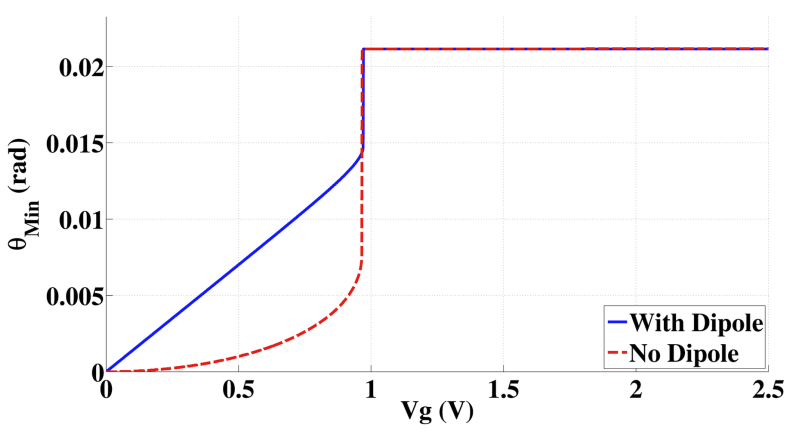

Figure 3.8: Comparison of the model with the addition and removal of the dipole moments.. With the additional of dipole moments to the model the switching characteristics change. Without the dipole moment the pull-in happens at the $2 / 3$ of the gap, where as with the strong dipole moments, the transition becomes much more linear instead of parabolic and the sticking occurs at $1 / 3$ of the gap. The model captures the exactly the same switching behavior as in [12] when the dipole moment is not included and the same design parameters are used (shown as dashed.)

As known in the ion gates, the dipole moments are the main mechanism behind the steep switching behavior [64]. They move the ion channel gates steeply as the membrane voltage increases above a threshold voltage with applied external current.

In the Fig. 3.8, we are making a comparison between two systems in which at first the dipole moments dominates and in the second the electrostatic force dominates. For this test comparison, the cantilever dimensions are kept exactly the same, but the dipole unit is scaled per length of the cantilever to be large enough to demonstrate the physics better. In the case of electrostatic force dominating, the exact switching behavior is captured with using the same dimensions and parameters as in [12]. The switching behavior is parabolic until the pull-in took place at the $2 / 3$ of the original gap. The added stronger dipole moment makes the switching became much more linear and moves the collapsing at the almost $1 / 3$ of the original gap. In both the system, since electrostatic force is present the switching voltage does not change significantly, but the switching distance from the contact changes. This proves that the model proposed can capture both the larger systems where dipole moment is insignificant and the smaller systems where dipole moment is more present over the electrostatic force.

\subsection{Hysteresis}

When we execute the voltage-gated phase transition, the cantilever sticks to the drain. It is this sticking force of adhesion that makes the angle change abruptly and cause its subthreshold swing to 
plummet. However, this means that for a reverse cycle, we will need to tear the cantilever away from the drain by catapulting it with a high negative gate bias out of the deep VdW potential back to the weaker parabolic potential. This adhesion creates a strong hysteresis loop, which bodes ill for subthreshold swing, because the average slope over the loop becomes quite large even though the walls of the loop remain steep.

Before we address ways to mitigate this loop, let us first discuss how this hysteresis will arise out of our equations. Note that the entire physics is governed by a gate voltage driven transition from a metastable to a stable state. For the forward swing, we move from a weak elastic potential to the van der Waals, while for the reverse, we need to wait till the gate voltage raises the van der Waals bottom substantially in order to exceed the bottom of the elastic potential. It's evident however, that if the voltages are varied very slowly near steady-state, i.e., given ample time, the cantilever would spontaneously stick by bending over (finding its global minimum given infinite time), and thereafter the hysteresis would disappear. It is therefore imperative to assume a finite scan rate for the gate voltage $V_{G}(t)$, and calculate assuming that the time constants of the cantilever are slower than the scan rate of the gate voltage. While this is trivially true in most experiments, in our model, this means we can no longer assume an equilibrium Boltzmann distribution $P(\theta) \propto e^{-\beta \theta}$ but solve the Fokker-Planck equation with a time dependent gate voltage to extract the non-equilibrium behavior of the cantilever including its hysteresis.

Thermal averaging over all possible angles captures the movements of the particles given an infinite amount of time to relax. Given infinite relaxation time, the particles can move to the lower energy point in the potential profile and reside there. This single switching voltage where either particles shift from the local minima to global minimum or reverse, removes the hysteresis. If we introduce the speed of the electron through the scan rate of the top-gate into our model, we start to observe hysteretic behavior once again shown in Fig. 3.9.

The Fokker-Plank equation coupled with scan rate dependent numerical solution of the Landauer's theory allowed us to capture the movement of the particles given a relaxation time of particles between the top-gate voltage scanning. In Fig. 3.9, we show the distribution of electrons movement with two respectable scan rates: the slow scan rate (see Fig. 3.9A) where the particles keep residing in the local minimum of the potential profile and the fast scan rate (see Fig. 3.9B) where the particles lag behind the potential profile. These two residing and lagging behind movement of the particles 

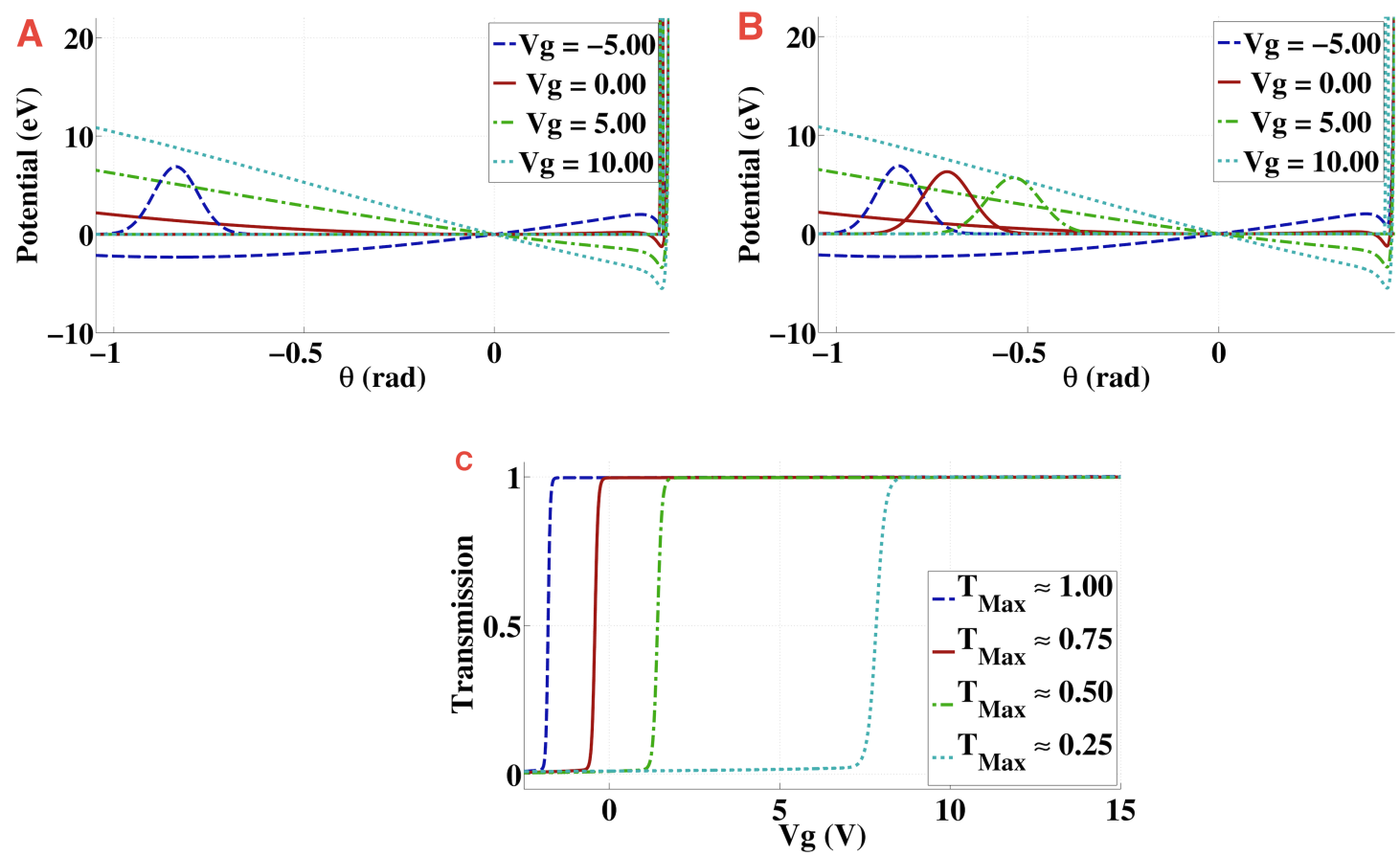

Figure 3.9: (C) The numerical solution of the Fokker-Plank equation coupled with the Landauer's theory results in switching voltages that are dependent on the scan rate. As the scan rate $\left(1 / T_{\max }\right)$ increases, the switching voltages shifts to right, more positive values, causing a hysteresis. (B) When the scan rate is fast enough, the particles do not have time to follow the potential, resulting in a delayed version of the Boltzmann distribution. $T_{\max }=1$ represents the case with no hysteresis where the scan rate and the movement speed of the particles are comparable (Particles can follow the rate of change in the potential) (A). $T_{\max }=0.25$ has a faster scan rate than the particles can follow resulting in a delay in transition causing it to switch at higher gate bias.

are why the Hysteresis occurs in the microscopic systems. As the scan rate increases the switching voltage delays further due to the particles lagging farther behind.

This microscopic model of hysteresis can be generalized for any system that have three different time scales: fast, slow, and intermediate. Hysteresis occurs when the transition occurs in the intermediate time scale between the fast and the slow. In the case of the NEMFETs, the slow time scale is the particle relaxation speed and the fast time scale is the maximum possible value of scan rate of the top-gate (giving electrons almost no time to relax or drift with the potential profile). When the top-gate is driven with a slower scan rate than the speed of the particles, the particles follow the same path way as the comparable scan rate then the speed of the particles, making no change in the switching voltage. However, as the scan rate speeds up to an intermediate scan rate value, the switching delays more and more causing a scan rate dependent switching voltage shown previously in Fig. 3.9C. 

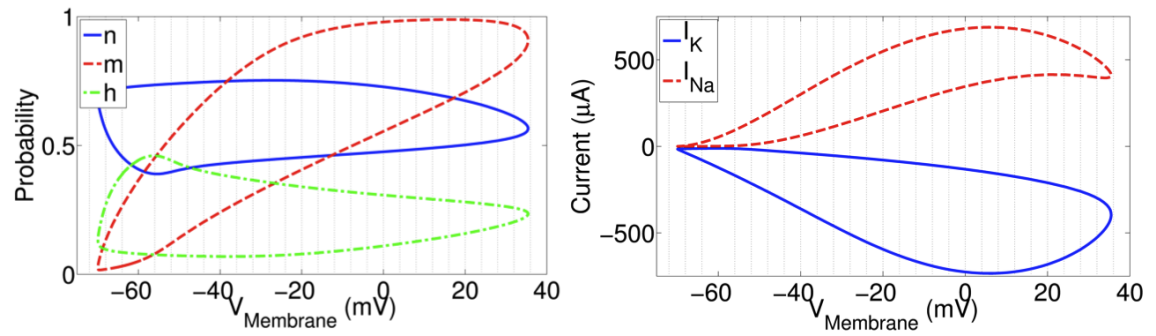

Figure 3.10: When the switching probabilities of the ion channels are investigated in membrane voltage versus probability rather than the conventional time versus probability, there is a distinguished hysteresis loop in all the switching probabilities ("n", "m", and "h"). These probability loops also result in a hysteresis loop in the sodium and potassium currents. These loops keep repeating themselves every time the axon compartment fires a new action potential.

\subsection{Connections to the Voltage-Gated Ion Channels}

One of the key features of the ion gates is the naturally occurring hysteresis seen when the gates are visualized in terms of voltage versus current rather than the conventional voltage versus time. The turning ON of the sodium channel is the initial channel to open, as shown with the red dashed curve in Fig. 3.10. The green-dashed curve represents the shutting down of the sodium channel, so that the net sodium ion flow could be stopped. As seen in the figure, there is a hysteresis in all the gating variables (presents the opening and closing of the proteins in the ion channels) since they are similar to the nano-mechanical relays modeled in this chapter. The attaching and detaching voltages differ, thus giving the final sodium current with a much more hysteretic loop. Additionally, hysteresis exists in the potassium current, but because it is modeled as only having one relay (see Fig. 1.7 for the closed form of the potassium channel), the shape of the hysteresis in the current is similar to the potassium gating variable.

When the flow of the sodium ions combines with the flow of the potassium ions, they form the shape of the action potential and we identify a clear hysteretic loop that is similar to seen in the nano-mechanical-relays. Fig. 3.11 shows the similarity by plotting both devices in terms of current versus voltage. One of the main reasons as to why the shapes differ in both hysteresis loops is the potassium overshoots the resting potential and hyperpolarizes the axon. For this reason, the upper part of the hysteresis loop in the action potentials almost have zero current before suddenly spiking upwards. The action potential has hysteresis because of the dipole forces in the cantilever shaped proteins. In the NEMFETs, hysteresis is due to the attractive van der Walls forces and the dipole forces. Because of this, each NEMFET should be used to model individual sodium and potassium ion channels in the axon. 

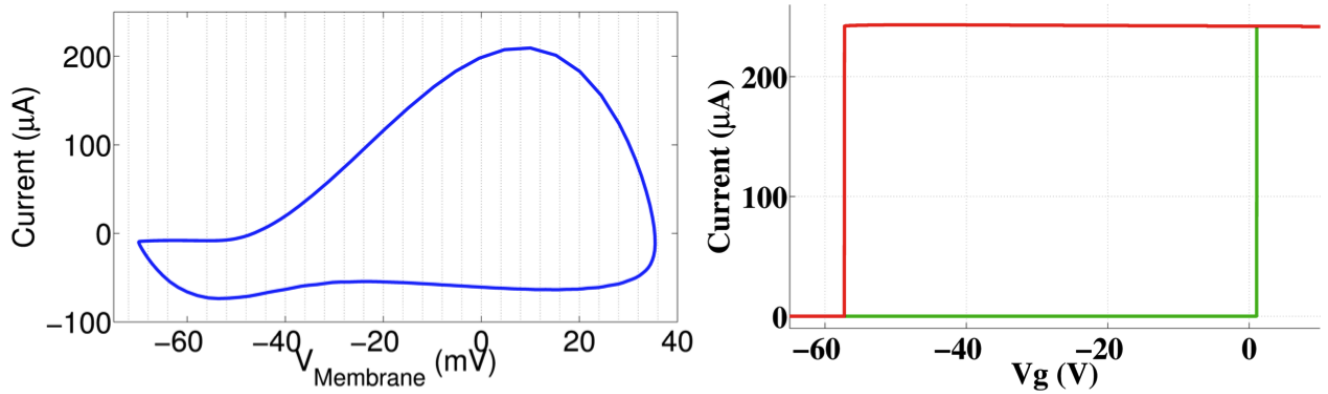

Figure 3.11: Comparison of the action potential hysteresis versus NEMFET hysteresis. Because of the overshoot of the potassium gates in the axon (dead-time), the loops differ in shape. The hysteresis is seen in both systems when plotted in current versus voltage. In the NEMFETs, the hysteresis occurs due to the different attaching and detaching voltages (due to the van der Walls forces). In the action potentials, it occurs due to the individual hysteresis loops in the sodium and potassium currents. NEMFET currents would be a better comparison with a single ion channel (sodium or potassium), since the turning $\mathrm{ON}$ and $\mathrm{OFF}$ voltages differ similar to the nano-mechanical relays.

By comparing the steepness of the conductances of both the nano-mechanical relay and the sodium conductance, we can identify that their subthreshold slopes are comparable. The fast switching sodium ion channel has a subthreshold slope of $7.5 \mathrm{mV} /$ decade [3] where as the modeled NEMFET has $6.5 \mathrm{mV} /$ decade slope (see Fig. 3.12). The NEMFET is one of the few solid-state components that can achieve such low steep switching characteristics, proving them to a great candidate to model the ion channels especially when using the model that incorporates dipole effects. The dipole model switching is significantly more similar to the ion channels because the protein relays change shape due to dipole moments rather than electrostatic forces.
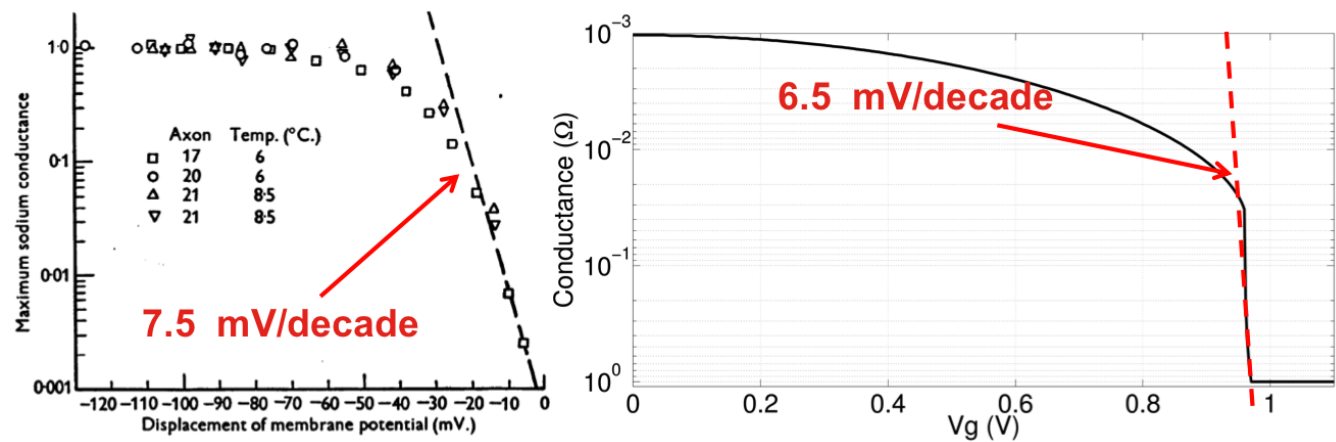

Figure 3.12: Matching of the steep switching in the voltage-controlled sodium ionic channels [3] and the nano-mechanical relays. With the use of dipole forces changing the shape of the proteins allowing passive transport through, the sodium channels can switch with almost $7.5 \mathrm{mV} /$ decade. If we use the nano scale dimensions where the dipole forces are much more prominent than the electrostatic forces, we achieve comparable steep switching of $6.5 \mathrm{mV} /$ decade in nano-mechanical relays. This proves that the NEMFETs can be used to model the ion channels and demonstrates their steep and low power switching behaviors. 


\subsection{Limitations and Challenges}

This chapter demonstrates how nano-mechanical relays are able to switch with a very sharp slope, and how the OFF current can essentially be zero. However, there are a few critical concerns that can still limit the overall performance of the nano-mechanical relays. The hysteresis loop in the fabricated large nano-mechanical relay devices (in micron sizes) and the smaller dimension biological and molecular components (in nanometer sizes) vary; even though the subthreshold slope is excellent in both sizes. This major difference should not be ignored when scaling the devices to smaller sizes. Since the capacitive forces are weaker in the smaller devices, the effect of the VDW forces is more dominant than in the larger devices. These larger devices that have bigger capacitive area lead to stronger capacitive forces. As the devices are aggressively scaled to nanometer sizes, the nano-cantilevers develop wider hysteresis loops, thus effecting the performance in electronics. While the devices are turning ON, the subthreshold swing will remain the same, however the average turn OFF subthreshold swing will enlarge as the pull-out voltages increase. This large increase in the pull-out voltage will increase the dynamic dissipation by orders of magnitude since it will affect the scalability of the supply voltage. This is where the dipole forces might help to improve the performance of the devices as shown in the Fig. 3.8.

As the devices scale down in size, their elasticity changes significantly. The smaller the cantilever length, the harder the cantilever is to bend, thus the width of hysteresis increases. One significant method of reducing this effect is to scale down the width of the cantilever as the length gets shorter. Although the width scaling helps with the elasticity of the cantilever, it causes the ON-current to proportionally scale with the width, thus once again affecting the performance of these nano-mechanical devices. Also, the surface adhesion forces do not scale in terms of van der Waals, but become more evident as the devices shrink to nanoscale dimensions.

A major limitation for nano-mechanical relays in high performance electronics is their switching speed in comparison to that of CMOS devices. The speed of relays is limited by a mechanical delay instead of capacitive charging or discharging. The mechanical delay in these relays is limited by the turn-on delay because the cantilever has to travel the physical air gap in order to make contact with the drain. The circuit speeds of the fabricated relays are in the orders of $\mathrm{MHz}$, where state-of-the-art CMOS devices can function in $\mathrm{GHz}$ regime. It is possible to improve the switching speeds of these devices by shrinking the air gap dimension, but then the elasticity and the surface adhesion problems 
become more prominent, as described. Innovative material and device engineering may be able to circumvent these limitations of scaling, speed, and hysteresis.

\subsection{Conclusions and Contributions}

Recently, hysteresis has attracted a lot interest from the neuroscience community due to its similarity to the neurons and synapses of the brain. The hysteretic and steep switching behavior of the nano-mechanical relays can be used to model the ion channels in the nervous system, allowing the creation of circuits that can mimic the performance of axonal networks.

In this chapter, a physics based microscopic modeling of the nano-electro-mechanical relay is presented. Our model captures important device behaviors, including the pull-in and pull-out voltages, low sub-threshold slope, and considerable $I_{\text {on }} / I_{\text {off }}$ ratios. The importance of the adhesive forces, van der Waals forces, and the length of the cantilever is identified and their effects on the low subthreshold slope are demonstrated. We show a numerical methodology of solving the gate voltage and time dependence and introduce the connections between the gate voltage scan rate and hysteresis. Finally, this chapter introduces a top level comparison of the current-voltage characteristics of nano-mechanical relays and ion channels. 


\section{Chapter 4}

\section{Conclusion}

\subsection{Summary}

The purpose of this dissertation is to develop and understand the physics behind the operation of the nervous systems' low power components (with specific focus on the elements used in the generation of action potentials in the axonal networks) through modeling electronic ratchets and nano-electro-mechanical relays. In the absence of global potentials, the ratchet-like mechanisms of the nervous system move ions by using flashing asymmetric potentials, powered by non-equilibrium conditions. The axonal network consists of switches that are similar to those found in relays as they have steep switching and hysteresis characteristics that generate electrical signals in the axon.

To extend the biological concept to a solid-state device, electronic ratchets are modeled using an AC clock and a series of potentials created by interdigitated electrodes. The non-equilibrium electronic ratchet is modeled in both the classical and quantum limits. This work proves how such a flashing ratchet device can be cascaded to create universal Boolean logic. Simulations and analytical derivations are used to explain any possible energy-saving advantages arising from reduced dynamic and static dissipation as in the low-power ion pumps. We outline limitations exposed by the simulations of our studied device geometries, namely, the importance of switching to current-driven (as opposed to voltage-driven) logic. Voltage-controlled current sources can reduce the dynamic dissipation that is associated with charging and discharging capacitors to almost zero. Finally, this work identifies the connection between electronic ratchets and low-power ionic pumps in the axonal networks by demonstrating how both devices take advantage of non-equilibrium conditions and utilize 
a symmetric potential to operate power efficiently.

A physics based microscopic model of the nano-electro-mechanical relay is presented to improve understanding of the steep switching. By using our model, important device behaviors are captured, including the pull-in and pull-out movements, low subthreshold slope, and considerable $I_{\text {on }} / I_{\text {off }}$ ratios. The importance of the adhesive forces, van der Waals forces, and the length of the cantilever is identified and their effects on subthreshold slope are characterized. We identify the results of both the dipole and capacitive based switching on the elasticity of the cantilever and analyze for switching performance. A numerical methodology for solving the gate voltage and time dependence is demonstrated and connections between the gate voltage scan rate and hysteresis are introduced. This work summarizes the limitations identified by the model, the effects of adhesive, dipole, and capacitive forces on scaling of the cantilevers. Finally, possible connections between the neurons and the nano-mechanical relays are investigated in terms of steep switching and hysteretic behavior.

\subsection{Future Research Extensions}

\subsubsection{Bio-Inspired Learning with Nano-Mechanical Relays}

For future research, multiple investigations based on the models developed in this dissertation can be used to extend the understanding of the neural networks. A significant study would implement the biologically-inspired learning devices using the hysteresis in the nano-mechanical relays. Simple synaptic devices with a spike-timing-dependent synaptic plasticity (STDP) learning function can

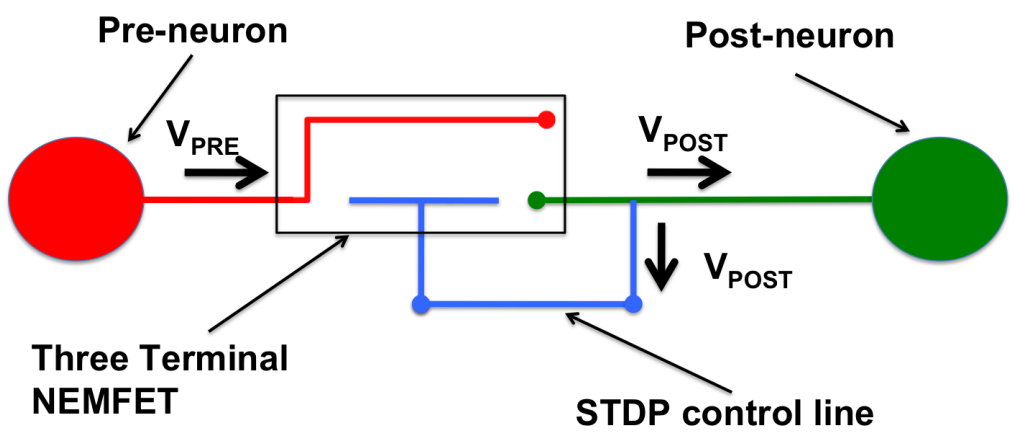

Figure 4.1: Control flow of the synapses composed of a three terminal nano-mechanical relay. The naturally occurring hysteresis in a current-voltage relationship can be utilized to implement the STDP learning mechanism. The all-or-nothing change in the conductance of the channel by applying a gate voltage can aid the system in memorizing conductance and can be maintained without fluctuations. Pre-voltage and post-voltage signals can be controlled by using the circuitry shown in [13]. 

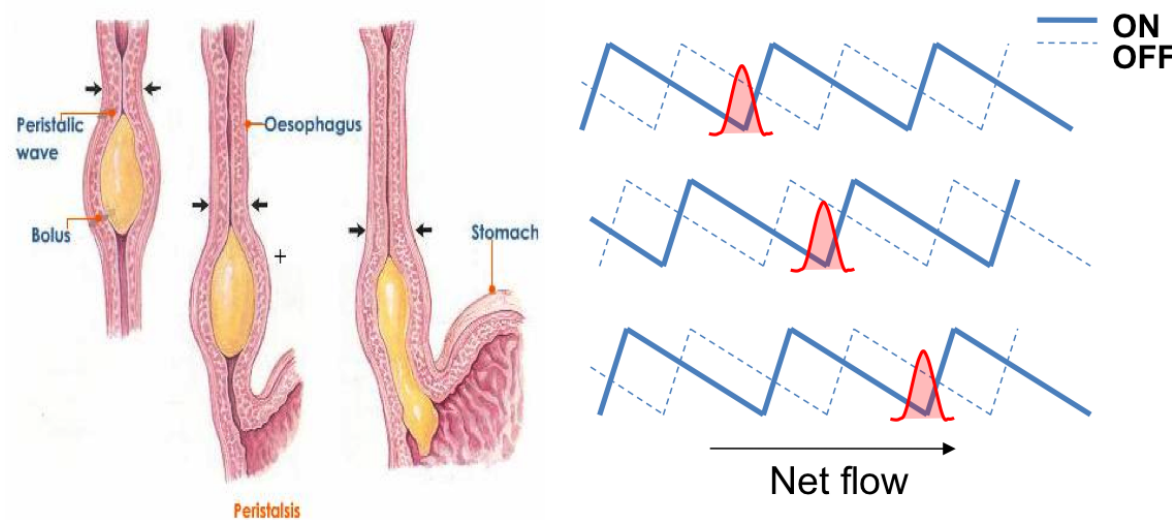

Net flow

Figure 4.2: This figure shows the peristaltic movement wave of the esophagus during swallowing, which allows the food to move in one direction. A possible implementation of peristalsis on the electronic ratchets is using multi-phase clocks (The turning ON of the first clock is followed by the turning ON of a phase-shifted second clock as the first clock turns OFF). This multi-clock system allows a net flow to the right with minimal back-flow of particles. Since the particles always drift from one well to the next and do not have time to diffuse equally, the ratchets in smaller scales can be implemented without the need of $\mathrm{THz}$ clock frequencies.

realize brain-like processors. The STDP is the learning mechanism of the mammalian brain synapses. A few studies have implemented this concept with the memristors in the form of a neuristor (memristor in series with a capacitor), but because of the steep and low-power switching arguments made in this thesis, the nano-mechanical relays can be a better replacing element and should be further investigated (See Fig. 4.1). Also, a more intensive mapping of these nano-mechanical relay equations to the widely known and accepted Hodgkin and Huxley equations can provide further insights on developing lossless long distance communications and fire action potentials in circuit level simulations. In theory, combining low low power ratchets and the steep switching nano-mechanical relays can enable long distance communications.

\subsubsection{Electronic Ratchet with Peristaltic Asymmetric Potential}

The performance (mostly speed) of the ratchets can be improved by implementing the peristalsis movement of the esophagus in addition to the asymmetric flashing potential. Using a wave-like pattern instead of the sin wave turn ON and OFF potential profile, the particles can be shifted directionally at lower clock frequencies, as shown in Fig. 4.2. The only problem with implementing this kind of design is the simultaneous timing of the clocks, which, like in CMOS, would affect the adiabatic switching properties of the ratchets. 

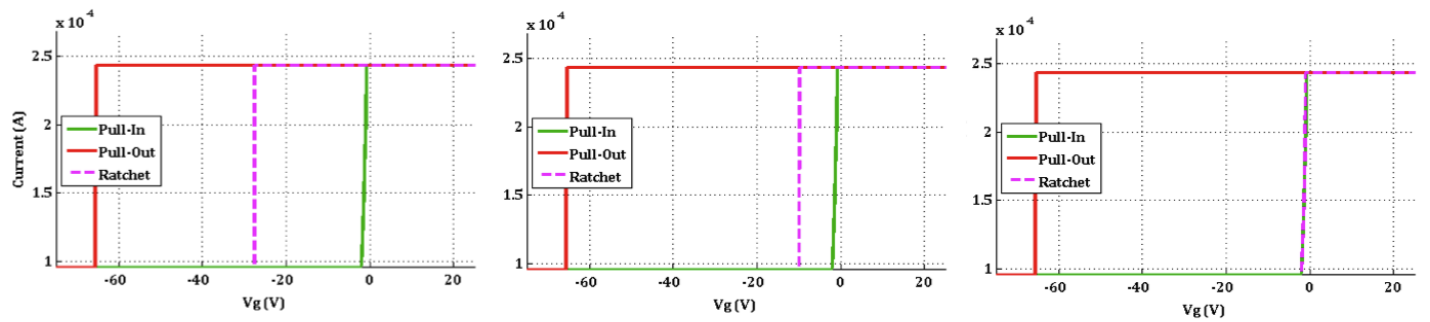

Figure 4.3: With the addition of the third contacts, the effect of the hysteresis begins to disappear as the voltage-bias is increased. Once the depth of the well is the same as the asymmetric potential of the third ratchet-like interdigitated contacts, the pull-in and pull-out are collapsed, thus enabling the use of nano-mechanical-relays for digital applications. As seen in the simulations above, the asymmetric potential removes the van der Walls well and enables the particles to drift back to the original potential well.

\subsubsection{Hysteresis Free Nano-Mechanical Relays}

An interesting continuation of this work could involve combining the nano-mechanical relay design with interdigitated contacts similar to the interdigitated ratchet designs. The third contact can help remove the hysteretic behavior of the nano-mechanical relays, thus making them a better candidate for high performance applications. In Fig. 4.3, we demonstrate this effect by adding three different ratchet potentials to the nano-mechanical relays. As the height of the asymmetric potential is increased with more applied bias, the hysteretic behavior begins to disappear. The pull-in and pull-out switching voltages become almost become the same when the height of the asymmetric potential matches the depth of the van der Walls well. With this potential design, both the stability of van der Walls and the low average subthreshold slope (being less than $60 \mathrm{mv} /$ decade required for high performance transistors) can be achieved. 


\section{Appendix A}

\section{Action Potentials: Analog Computing}

The efficient, optical propagation of signals is critical to a range of systems from underwater coaxial cables to neural system to nano-networks. The issues at hand are somewhat universal namely, transmitting the information at maximal bit-rate with minimal distortion, maintaining maximal fidelity, and minimal energy cost. While the solutions for large scale systems have been established over the decades of research, the trade-off alter drastically as we proceed towards smaller, nano scale systems. For instance, sending a signal at constant speed with a constant pulse shape requires both phase velocity and the attenuation to be frequency independent. Classical transmission lines can accomplish this using match filter, for instance, by inductively loading cable with a metal of high magnetic permeability. However, it is not practical for smaller systems such as for neural communication in the brain or the nano scale on chip interconnects. Thinning down the transmitting wires requires larger, more burdensome inductive loading elements, while simultaneously slowing down the signals, making computation quite inefficient.

In biology, after years of evolution and optimization, we do not face the same computation problems and limits that we encounter in the state-of-the-art CMOS circuits. Neural networks address these problems substantially different and quite cleverly by using series of switches and batteries to remove the signal decay and maintain the shape and velocity of the propagating signals. As we get into the nano scale CMOS dimensions, electron tunneling becomes one of the most significant device problems [1]. However in biology, due to the large ionic masses, no tunneling occurs, 


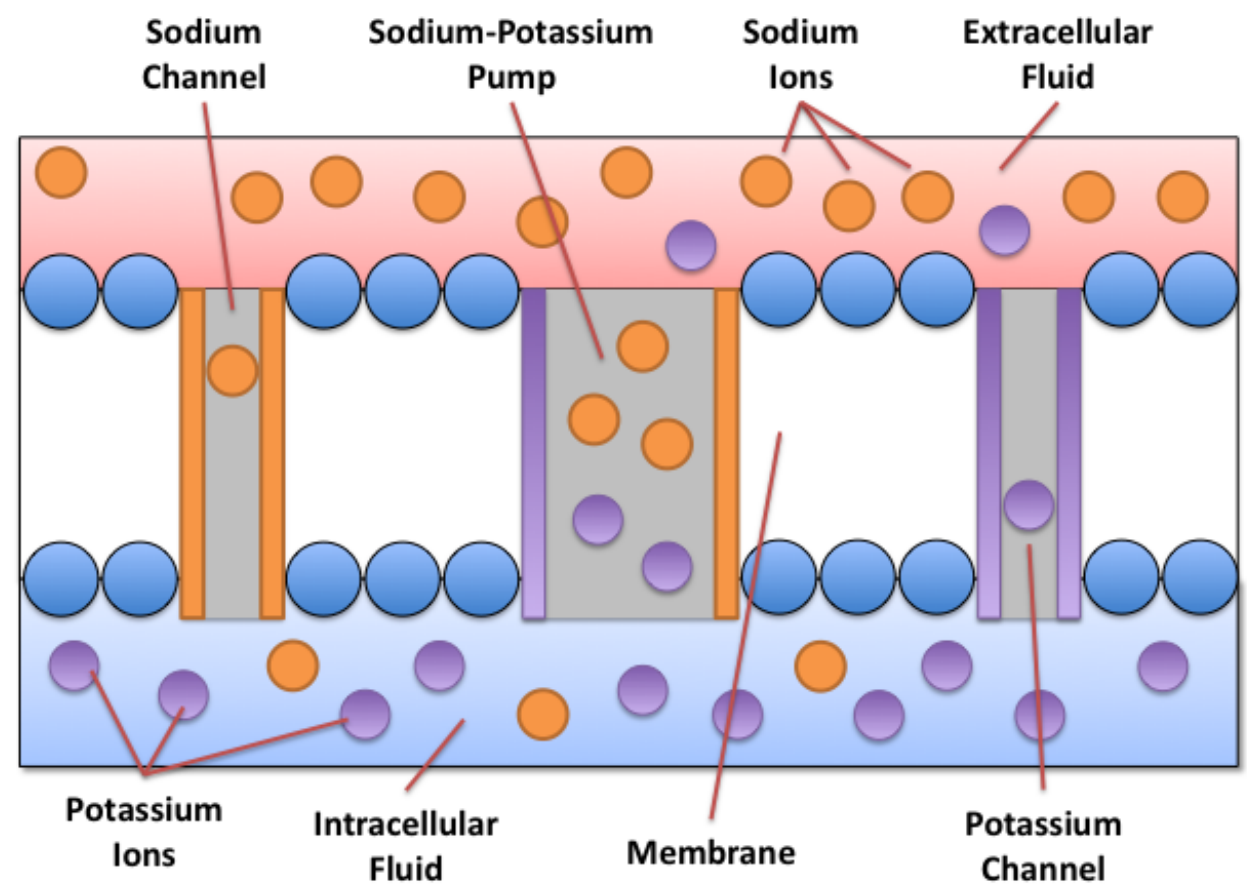

Figure A.1: The voltage-controlled potassium (purple) and sodium (orange) ion channels. They mechanically change shape when the sodium $\left(\mathrm{Na}^{+}\right)$rich positive potential on the outside from the potassium $\left(K^{+}\right)$rich potential on the inside of a axon is changed by an external pulse. They allow transport of the ions passively where as the adenosine triphosphatase based sodium-potassium ion pump within a cell membrane restores the membrane potential actively.

allowing fast and short distance communication [84]. Also instead of trying to minimize noise and thermal energy, neurons utilize them to maximize the performance as seen in neurons [85]. They parallelize communication by using flexible routing of interconnects (axons). These is almost similar to the way the industry is shifting towards to improve the performance of the modern computers. In the past, we were only scaling the sizes of the individual transistors, but in the last decade the multi-core processors are started to be used in order to parallelize the work load and improve the performance.

\section{A.1 Information Encoding in the Neurons}

In neural networks, traveling signals called the action potentials (APs) are the way neuron communicates information down an axon to another neurons. They are short-lasting electro-chemical events in which the electrical potential of a cell membrane rapidly increases followed by a decrease when excited by an external signal pulse. APs are also known as "nerve impulses" or "spikes". These analog signals are sent along transmission lines (axons), with non-linear repeaters (ion pumps and 


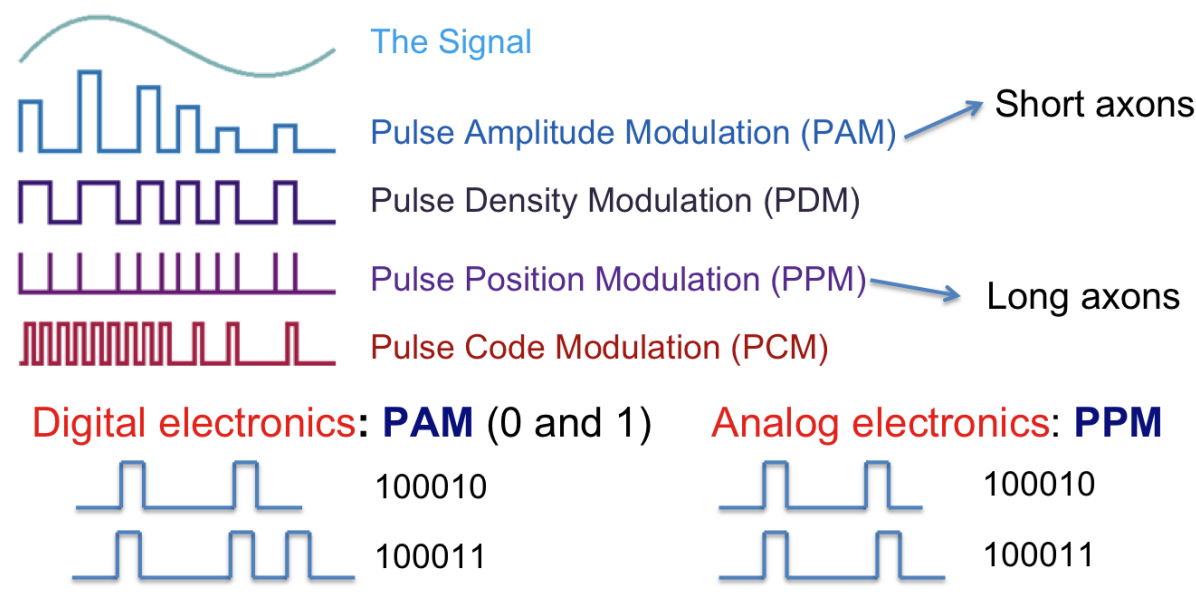

Figure A.2: The information (bits) in neural networks is stored between the time intervals of two successive electrical pulses. Neurons use this different differential pulse position modulation technique since it is the most power efficient way of encoding information. Two main ways to maximize the bit-rate per joule in the neural networks: First by squeezing the pulse width in the cost of increasing the overall cost of spike generation. Second by decreasing the time between two successive pulses in the cost of distorting the information stored between two pulses.

voltage-gated ion channels). They are all-or-none events, there is no such spike as a big or small AP. Their properties such as height and velocity are characterized by the axon they travel though. The ion pumps and voltage-gated channels allow the polarity of the inside of the membrane to change actively or passively as ions pass through them (Fig.A.1).

Information is stored and transmitted by using the pulse position modulation scheme rather than the pulse amplitude modulation in CMOS. Information is coded by using inter-spike interval (ISI) in

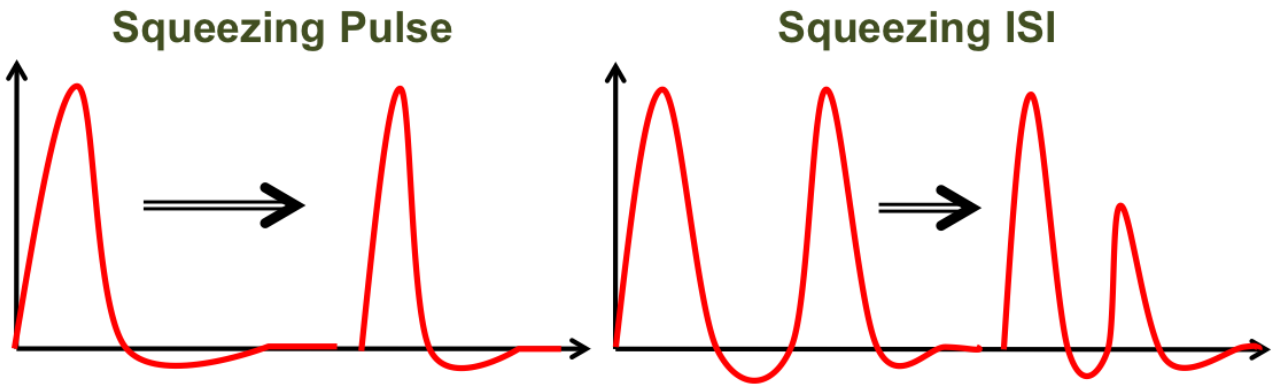

Figure A.3: The information (bits) in neural networks is stored between the time intervals of two successive electrical pulses. Neurons use this different differential pulse position modulation technique since it is the most power efficient way of encoding information. Two main ways to maximize the bit-rate per joule in the neural networks: First by squeezing the pulse width in the cost of increasing the overall cost of spike generation. Second by decreasing the time between two successive pulses in the cost of distorting the information stored between two pulses. 

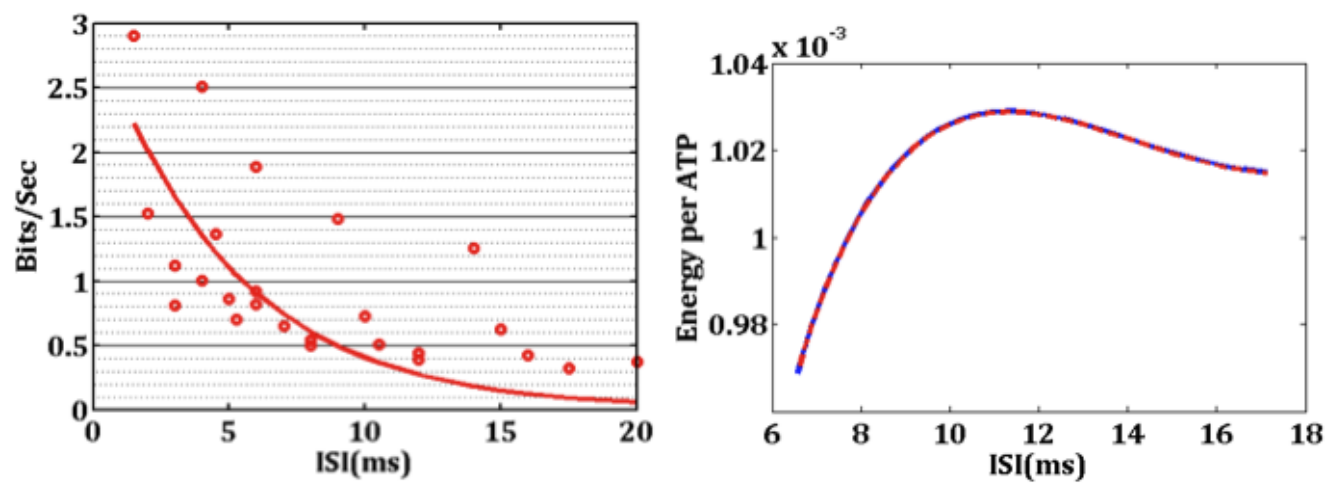

Figure A.4: Bits per second vs. ISI: As the time between two successive pulses increase, the number of Bits/Sec decreases assuming the gamma distribution with varying the mean. Shorter the separation between the more bits per second the axonal network can carry. Energy vs. ISI: As the time between two successive pulses increase, the energy of generating a spike changes also since the overlap of sodium and potassium currents differ. There is a peak point in which the cost of generating a spike becomes too expensive, so the neurons optimized by the axon properties avoid generating spikes at tho given ISI.

which it is stored in the time between two successive spikes (see Fig.A.3) [86, 87]. ISI coding can provide additional information with the same bit-rate since the frequency or the number of spikes increases as the intensity of the stimulus increases. Also, the cost of neural communication is determined by the velocity of the AP and the bit-rate is optimized when the energy costs are taken in account $[88,89]$.

The neural networks like to propagate as many signals as possible with a constant velocity. One way to maximize the bit-rate is simply to pack in successive pulses closely (Fig.A.3), but increasing the pulse to pulse overlap would distort their shapes to that trailing and leading edges cannot be discerned accurately to clock their arrival and departure. Alternatively, one can squeeze each individual pulse by increasing the overlap between the opposite sodium and potassium currents, thus increasing the total energy cost. The neurons operate with maximizing the bit-rate per joule with a given threshold on the distortion, and using the axon properties to construct the optimal pulse shape, by varying the separation between the sodium and potassium currents.

The average number of bits in the ISI is calculated by using Shannon's entropy, which quantifies the expected value of bits contained in a message. The Shannon's entropy is computed numerically using a gamma distribution with varying the mean [90]. Below a certain minimum time interval between spikes and a certain time required for the axon to get back to resting potential is called dead-time. Information is distorted and can not be stored or transmitted. The maximum message 
rate is limited by the absolute dead-time while the information per pulse will be controlled by the probability distribution of a pulse. Fig.A.4 shows that as the separation between two spikes increase, the number of bits/sec decreases. As the ISI time changes, the overlap of the sodium and potassium currents differ, thus impacting the cost of successive pulse generation. When the energy per adenosine triphosphate (ATP) is calculated by extracting the area of the sodium and potassium currents overlap (Fig.A.4), the cost of generating a single spike can be calculated. There is a very important relationship between the velocity of a spike and the ISI separation (Fig.A.5) [88]. Due to the distortion of the signals as pulse separation decreases, the velocity of the spikes change [91]. For slower action potentials, the overlap increases thus costing more the spike generation. The neural networks optimize the energy of the spike generation by changing the ISI time to get the maximum bit-rate per joule.

\section{A.2 Physics of an Action Potential}

Understanding the signal generation in an axonal system requires delving into the individual current components comprising the signal. The action potentials are initiated and transmitted through an axon with the help of the voltage-controlled ion channels and ionic pumps [41]. When not firing a signal, the lipid bilayers separate a sodium rich positive potential on the outside from a potassium rich negative potential in the inside (Fig.A.6). This potential difference across the membrane at rest is called the resting potential and with using the Nernst equation can be explored. The Nernst equation considers the valence, outside, and inside concentrations of the ions being considered. In a typical axon at start, both the sodium and the potassium the channels are shut when the membrane potential is at resting potential. When an external signal in the form of a traveling wave arrives and it increases the
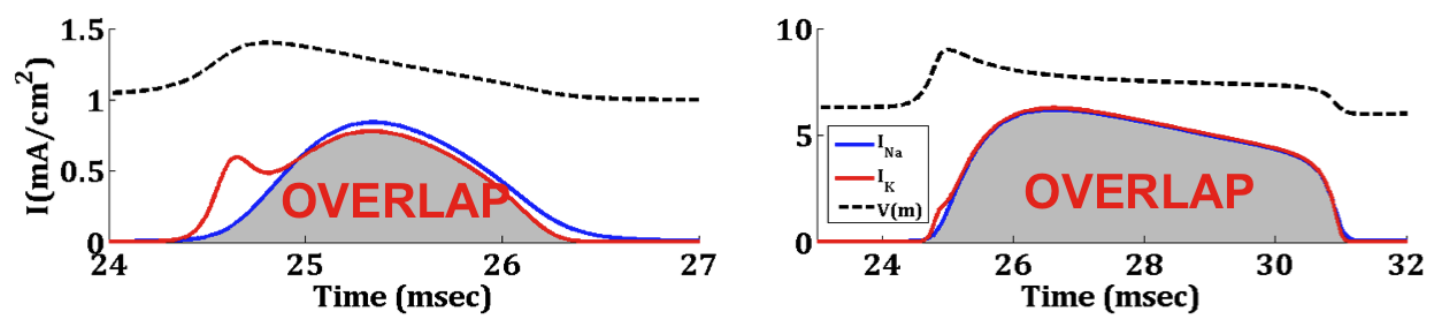

Figure A.5: As the time between two successive pulses increase, the velocity of the action potential generated changes due to the distortion between the two signals. This change in the velocity has a huge impact on the energy of generating a spike changes since the overlap of sodium and potassium currents differ. As seen in the figure, for a slower spike, the overlap increases thus the overall cost of generating single spike goes up. 


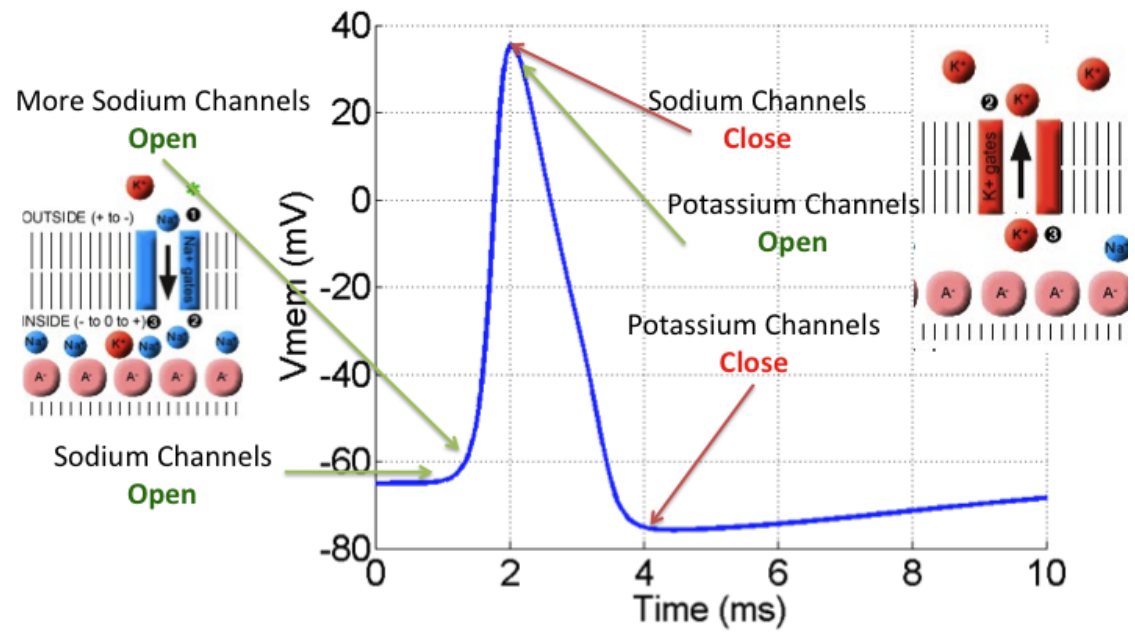

Figure A.6: Action potential spiking mechanism: Opening and closing times of the sodium and potassium channels during the initiation of an action potential. First the sodium channels are activated and followed by the potassium channels to create a traveling spike.

membrane voltage in the amounts of a predefined threshold voltage. The threshold voltage is a sum of potential decreases at the axon hillock and that's why the action potentials are all-or-nothing events.

The increased voltage difference between the inside and outside of the cell, generates an electromagnetic field strong enough, that it induces a conformational change in the potassium channel. The membrane voltage change distorts the shape of the channel protein (the relay) enough that a physical cavity/gap opens and admits the $\mathrm{Na}^{+}$ions influx to occur across the membrane. The sodium ions want to move from a high concentration to a low concentration to create equilibrium, so they flow inward, further increasing the membrane potential and opening even more sodium channels. The opening of all the available sodium channels results in an upswing of the membrane potential. This stage is defined as the "depolarization" which means a decrease in the absolute value of the membrane potential. This rapid influx of sodium ions reverses the polarity of the membrane, causing the sodium channels to start deactivating rapidly. As the sodium channel closes, the sodium ions can no longer flow inward passively and they are need to be actively transported out of the cell membrane by the ion pumps.

The potassium ion channels are activated the same way as the sodium channels by mechanically moving the relay allowing an outward flow of the $K^{+}$ions. This stage is called the "repolarization" where the absolute value of membrane potential increases. The electrochemical potential returns back to resting value, but since the potassium channels do not respond immediately to their voltage-gated 

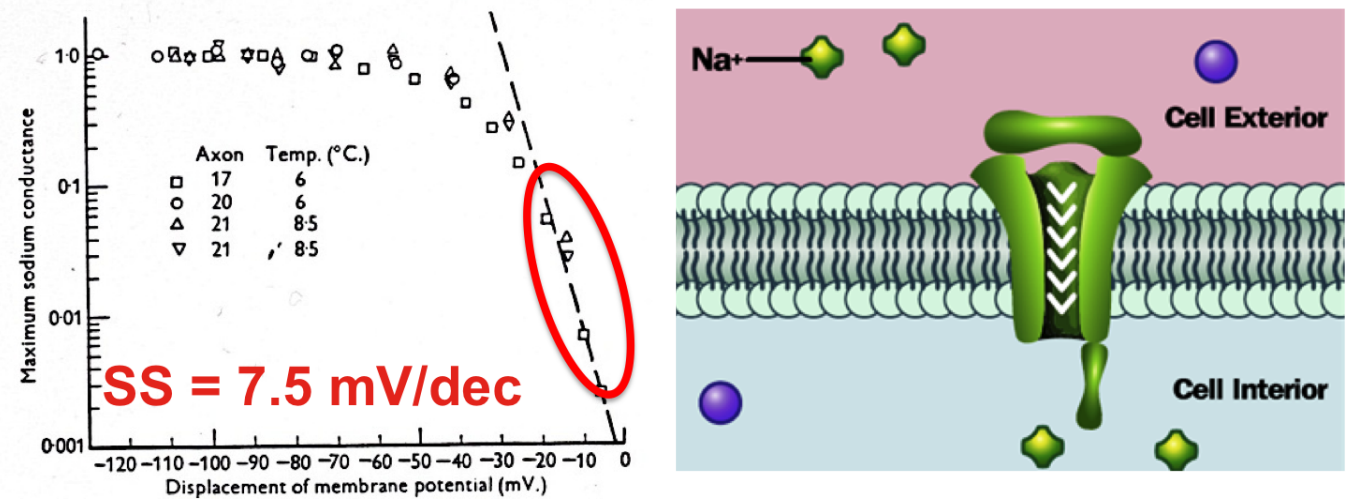

Figure A.7: Ultrafast potassium channel activation of $7.5 \mathrm{mV} /$ decade from the Hodgkin and Huxley experiments. The channel goes under a conformational change to create a physical gap for the ions to pass through the channel membrane.

properties (they are time-dependent as well), thereby overcompensating and "hyperpolarizing" the channel. This undershoot is expected before the membrane settles back to rest. In general, since the resting potential of a neural membrane is negative, signals take the form of increasing to a more positive value.

Ultimately the channels (switches) close, and a series of sodium-potassium pumps (battery) opens and continuously move ions against the their concentration gradient actively by using energy-storing molecules called ATP. The ATP synthesis actively pumps $3 \mathrm{Na}^{+}$ions out of the cell, at the same time pumping $2 \mathrm{~K}^{+}$ions into the cell to restore the ionic gradient. This transient negative shift in the membrane potential due to the additional potassium currents is called the dead-time. The net outcome is a voltage pulse with a characteristic shape that propagates directionally along the axon, alternatively opening and closing the switches and batteries. This system is modeled well empirically as a traveling wave driven by a series of nonlinear voltage-gated conductances that help charge the capacitor. Another very significant property of the dead-time is it is almost impossible to generate action potentials in the dead-time [91].

\section{A.3 Hodgkin and Huxley Model}

Alan Hodgkin and Andrew Huxley began a series of experiments to visualize the ionic current flow in the channels and to represent the channels opening and closing mechanism with mathematical equations. They used a voltage clamp, which is a tool that converts ionic current to 


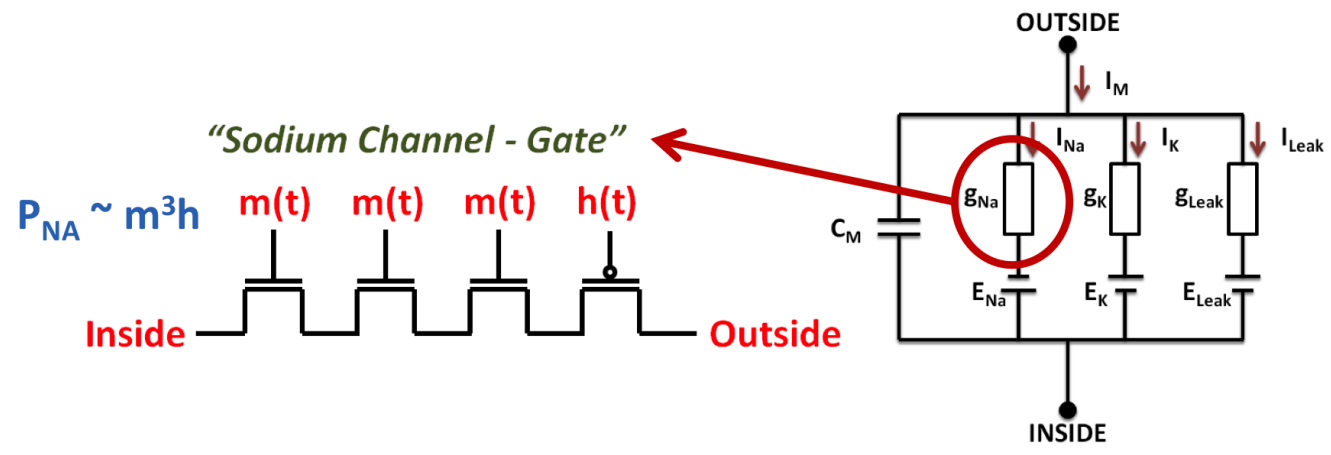

Figure A.8: The Hodgkin and Huxley electrical equivalent circuit for a squid giant axon. The capacitor represents the capacitance of the cell membrane; the two variable resistors represent voltage-dependent $\mathrm{Na}^{+}$and $\mathrm{K}^{+}$conductances, the fixed resistor represents a voltage-independent leakage conductance and the three batteries represent reversal potentials. The probability of opening and closing of the ion gates are similar to the FETs. Sodium gate is regulated by $m(t)$ like a NMOS and $h(t)$ like a PMOS. $m(t)$ to third exponent represents the fast opening of the sodium gate where as $\mathrm{h}(\mathrm{t})$ represents slow shutting of the sodium gate.

electrical current. This allowed them to probe the cell and measure the transmembrane potential while applying a constant voltage to the cell (Fig.A.7) [92, 93]. With the constant voltage, they can measure the ionic conductance changes without the influence of the voltage dependent parameters.

Using the voltage clamp, Hodgkin and Huxley applied various transmembrane voltages and recorded the changes in the ion currents and started to identify the signs of an action potential. Between certain ranges of potential, there was an odd shift in the ionic currents and by blocking the either one of the sodium or potassium using tetrodoxin or tetrathylammonium, they were able to plot the individual ionic currents.

In 1952, they published series of articles, which introduced the basic processes underlying the communication between nerve cells [92]. Their research was based on electro-physiogical experiments on a giant axon squid to understand how action potentials in neurons are initiated and propagated. They developed series of mathematical equations (Hodgkin and Huxley model), which approximated the electrical characteristics of individual ionic currents by using the a relationship between conductance and transmembrane voltage. They developed a simple circuit made of only capacitors, resistors, and batteries to mimic the cell membrane and the ionic currents (Fig.A.8). 


\section{A.3.1 Hodgkin and Huxley Circuit and Empirical Equations}

Hodgkin and Huxley modeled the semi-permeable cell membrane as a capacitor, which is separating the inside of the cell to outside [92]. If an external current were injected to the system, it would either add further charge to the capacitor or leak through the cell membrane into the cell, causing a concentration gradient between inside and outside. The capacitive currents across the cell membrane can be described as the sum of changes in the voltage across the membrane $\left(V_{m}\right)$ and ion currents caused primarily by sodium and potassium and other leakages. The leakage currents take care of other channel types that are not modeled explicitly like the chloride. The ion currents are defined by their conductance $\left(g\right.$, with the $\mathrm{Na}^{+}$and $\mathrm{K}^{+}$conductance being voltage depended), their equilibrium potentials $(E)$ and the probability of the channel gates being open or close $(m, n, h)$.

$$
\begin{aligned}
I_{\text {ext }} & =C_{m} \frac{d V_{m}}{d t}+I_{i o n} \\
I_{\text {ext }} & =C_{m} \frac{d V_{m}}{d t}+\underbrace{g_{N a} m^{3} h}_{G_{N a}(V, t)}\left(V-E_{N a}\right)+\underbrace{g_{K} n^{4}}_{G_{K}(V, t)}\left(V-E_{K}\right)+\underbrace{g_{L}}_{G_{L}}\left(V-E_{L}\right)
\end{aligned}
$$

The equations A.1 shows the conservation of the electric charge in the membrane, once a current is applied, it can either add further charge to the capacitor or pass through the specific ion channels. The standard Hodgkin and Huxley model expands into a set of four differential equations that are fitted from the experimental data of 1952:

$$
\begin{aligned}
\frac{d m}{d t} & =\alpha_{m}(V)(1-m)-\beta_{m}(V) m \\
\frac{d h}{d t} & =\alpha_{h}(V)(1-h)-\beta_{h}(V) h \\
\frac{d n}{d t} & =\alpha_{n}(V)(1-n)-\beta_{n}(V) n
\end{aligned}
$$

The Hodgkin and Huxley equations only include three types of channel, which are characterized by their conductance: sodium, potassium, and leak (chloride). The leakage channel has voltageindependent conductance, where are the sodium and potassium conductance is dependent on both voltage and time. If all channels are open, the channels transit ions with maximum conductance of $g_{N a}$ and $g_{K}$, however in reality some of the channels will be blocked. To capture this behavior, Hodgkin and Huxley included opening probabilities that were controlled by the additional gating parameters of $m, n$, and $h$ (Eq. A.2). The combination of $m$ and $h$ controlled the ultrafast sodium channels and $n$ controlled the potassium gates (Fig.A.9). 

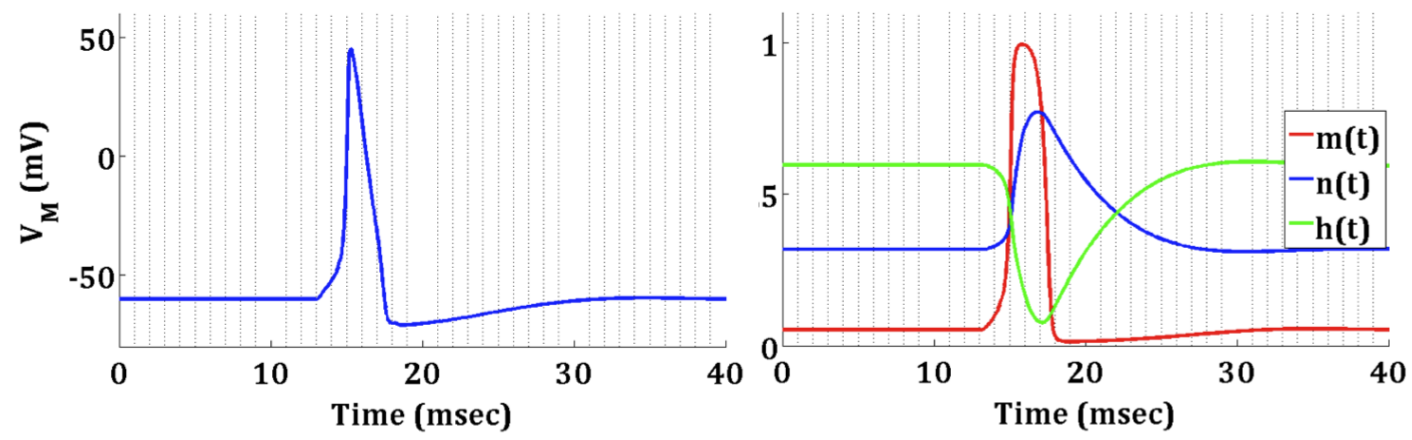

Figure A.9: The numerical solution of Eq. A.1 of the Hodgkin and Huxley model showing the membrane voltage vs. time after the axon is excited by an external pulse. The $m(t), n(t)$, and $h(t)$ show the probability of an ion channel opening and closing in time and Eq. A.2 and Eq. A.3 are used to calculate the values. The $m$ represents the probability of the fast-time scaled sodium channels opening where as the $n$ shows the potassium channel probability. $h$ demonstrate the slow time-scale closing of the sodium channels.

$$
\begin{array}{ll}
\alpha_{m}(V)=\frac{0.1(V+40)}{1-\exp \left(\frac{-(V+40)}{10}\right)} & \beta_{m}(V)=4 \exp \left(\frac{-(V+65)}{18}\right) \\
\alpha_{h}(V)=0.07 \exp \left(\frac{-(V+65)}{20}\right) & \beta_{h}(V)=\frac{1}{1+\exp \left(\frac{-(V+35)}{10}\right)} \\
\alpha_{n}(V)=\frac{0.01(V+55)}{1-\exp \left(\frac{-(V+55)}{10}\right)} & \beta_{n}(V)=0.125 \exp \left(\frac{-(V+65)}{80}\right)
\end{array}
$$

The three gating parameters are determined by the voltage dependent $\alpha$ and $\beta$, which are empirical functions that were adjusted by Hodgkin and Huxley to fit the experimental data of the squid axon in Eq. A.3. The equation for the each gating parameters can be rewritten as in a more generic form of

$$
\frac{d x}{d t}=-\frac{1}{\tau_{x}(u)}\left[x-x_{0}\left(V_{m}\right)\right]
$$

where $x$ stands for $m, n$, or $h$ and for a given fixed voltage $V_{m}$, the gating variable approaches to the value of $x_{0}\left(V_{m}\right)$ with a time constant of $\tau_{x}(u)$. This way a better comparison between the gating particles can be done, however it is still hard to develop an understanding in terms of electronics from these complicated and unphysical equations. 

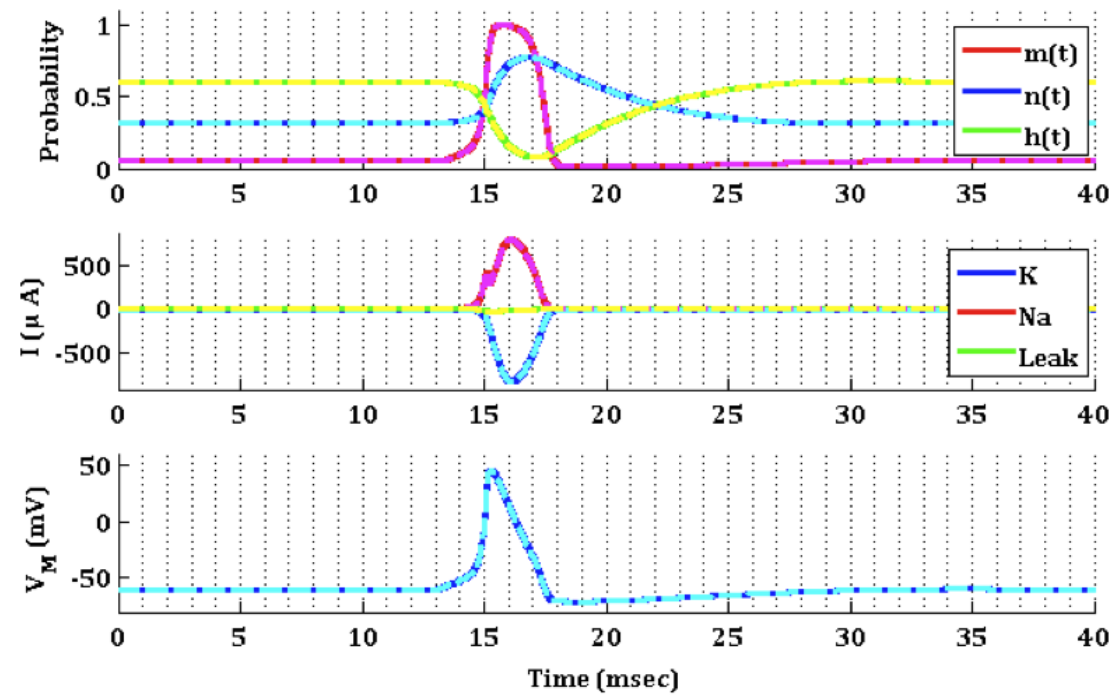

Figure A.10: The fitting of the rate constants with the Fermi-Dirac equations, resulting in a almost perfect fit of the current-voltage curves of a firing action potential. The individual currents for sodium, potassium, and leak and the gating particles $m(t), n(t)$, and $h(t)$ are with in one percent fitting tolerance when the Eq. A.5 is used instead of Eq. A.3.

\section{A.3.2 Fermi Function fitting of Hodgkin and Huxley}

Instead of using the unphysical exponential function for the $\alpha$ s and $\beta$ s from the original Hodgkin and Huxley equation, we used a simple well understood formula in electronics, Fermi-Dirac functions to fit the rate constants. The specific form selected to fit all the $\alpha$ s and $\beta$ s for $m, n$, and $h$ is

$$
f(V)=\frac{1}{\exp \left(\frac{V-v 0}{k T}\right)+1} * m t
$$

where the fitting parameters are $v 0, k T$, and $m t$ [94]. The $v 0$ accounts for the turning on voltage, $k T$ accounts for the speed of turning-on (thermal temperature), and the $m t$ is an outside multiplier to fit the $m$ values since their rate constants are not limited between 0 and 1 . The table A.1 shows the values selected for each gating parameter. In this form, the $k T$ is the most important parameter since it has a direct correlation to the turning on speed with the outside parameter $m 0$.

Table A.1: Fitting Parameters for Equation A.5

\begin{tabular}{|c|c|c|c|c|c|c|}
\hline & $\alpha_{n}$ & $\beta_{n}$ & $\alpha_{m}$ & $\beta_{m}$ & $\alpha_{h}$ & $\beta_{h}$ \\
\hline$v 0$ & -1.8 & -202 & 18.2 & -114 & -107 & -30 \\
$k T$ & -21 & 73 & -24.2 & 17 & 18.3 & -10 \\
$m t$ & 1 & 1 & 10.7 & 100 & 1 & 1 \\
\hline
\end{tabular}


The figure A.10 shows the matching of the Fermi-Dirac results with the original Hodgkin and Huxley equations. As seen from the figure, action potentials and ionic currents match significantly with the results although there are small mismatches in the heights of the action potential. These Fermi-Dirac results help us understand importance of the thermal temperature, the $k T$ parameter. From the derivatives of the $\alpha$ s and $\beta \mathrm{s}$, one can see that $m$ has the highest absolute slope, followed by the $n$ and $h$. Analyzing the parameters in table A.1 can also derive the same conclusion. The highest value out of the six fitted equations is as expected the steep sodium turning on. One important factor to point out is the $\alpha$ s and $\beta \mathrm{s} k T$ values differ from each other. This shows that the action potential is a non-equilibrium system which actually utilizes the thermal atmosphere surrounded by. 


\section{Appendix B}

\section{Publications}

\section{B.1 Journals}

- M. Kabir, D. Unluer, A. W. Ghosh, and M. R. Stan, Computing with Non-equilibrium Ratchets, IEEE Transactions on Nanotechnology, vol. 12 no. 3, pp. 330-339 (2013).

- D. Unluer, F. Tseng, A.W. Ghosh and M.R. Stan, Monolithically Patterned Wide-Narrow-Wide All Graphene Devices, IEEE Transactions on Nanotechnology vol. 10 no. 5, pp. 931-939 (2011).

- F. Tseng, D. Unluer, K. Holcomb, M.R. Stan, and A.W. Ghosh. Diluted Chirality dependence in edge rough graphene nanoribbon field effect transistors. Applied Physics Letters, 94(22):223112, (2009).

- D. Unluer and A.W. Ghosh, Subthermal switching with nanomechnical relays, (2013). in preparation.

\section{B.2 Book Chapters}

- F. Tseng, D. Unluer, M.R. Stan and A.W. Ghosh. Graphene Nanoribbons: from chemistry to circuits, Graphene Nanoelectronics: Metrology, Synthesis, Properties and Applications. Springer-Verlag, 2012. 


\section{B.3 Conferences}

- D. Unluer, F. Tseng, and A.W. Ghosh. Physics-based GNRFET compact model for digital circuit design, Semiconductor Device Research Symposium, (2011).

- M. Kabir, D. Unluer, L. Li, A. W. Ghosh, and M. R. Stan. Electronic ratchet: A non-equilibrium, low power switch, IEEE Nanotechnology Conference, (2011).

- L. Li, D. Unluer, M. Kabir, F. Tseng, M. R. Stan, and A. W. Ghosh. Switching limits in nano-electronic devices, IEEE Nanotechnology Conference, (2010).

- D. Unluer, F. Tseng, A.W. Ghosh, and M.R. Stan. Atomistic deconstruction of clear performance advantages of a monolithically patterned wide-narrow-wide all-graphene FET, Device Research Conference, (2009).

- F. Tseng, D. Unluer, M.R. Stan and A.W. Ghosh. Absolute Control of Chirality Unnessary for Wide-Narrow-Wide Graphene Field Effect Transistor, Device Research Conference, (2009).

- M. R. Stan, D. Unluer, A. Ghosh, F. Tseng. Graphene devices, interconnect and circuits challenges and opportunities, IEEE international symposium on Circuits and Systems, (2009).

- A. T. Barth, M. A. Hanson, H. C. Powell, D. Unluer, S. G. Wilson, and J. Lach. Body-coupled communication for body sensor networks, Proceedings of the ICST 3rd international Conference on Body Area Networks, (2008). 


\section{Bibliography}

[1] V.V. Zhirnov and R.K. Cavin. Microsystems for Bioelectronics: the Nanomorphic Cell. Micro and Nano Technologies. Elsevier Science, 2010.

[2] Darrell Sharp. Nerves, hormones and homeostasis, 2011.

[3] A. L. Hodgkin and A. F. Huxley. Currents carried by sodium and potassium ions through the membrane of the giant axon of loligo. J Physiol, 116(4):449-472, 1952.

[4] H. Linke, B. J. Alemán, L. D. Melling, M. J. Taormina, M. J. Francis, C. C. Dow-Hygelund, V. Narayanan, R. P. Taylor, and A. Stout. Self-propelled leidenfrost droplets. Phys. Rev. Lett., 96:154502, Apr 2006.

[5] Wikipedia. Pump and relay, 2013.

[6] Emma. Neuroscience: The action potential. Website, March 2013.

[7] Martin Bier. Brownian ratchets in physics and biology. Contemporary Physics, 38(6):371-379, 1997.

[8] M. Kabir, D. Unluer, Lijun Li, A.W. Ghosh, and M.R. Stan. Electronic ratchet: A nonequilibrium, low power switch. In Nanotechnology (IEEE-NANO), 2011 11th IEEE Conference on, pages $482-486$, aug. 2011.

[9] R. Ait-Haddou and W. Herzog. Force and motion generation of myosin motors: muscle contraction. Journal of Electromyography and Kinesiology, 12(6):435 - 445, 2002.

[10] R. D. Astumian and I. Derenyi. Fluctuation driven transport and models of molecular motors and pumps. Eur Biophys J., 27:474-89, 1998.

[11] N. Abele, R. Fritschi, K. Boucart, F. Casset, P. Ancey, and A.M. Ionescu. Suspended-gate mosfet: bringing new mems functionality into solid-state mos transistor. In Electron Devices Meeting, 2005. IEDM Technical Digest. IEEE International, pages 479 - 481, dec. 2005.

[12] K. Akarvardar and H.-S.P. Wong. Analog nanoelectromechanical relay with tunable transconductance. Electron Device Letters, IEEE, 30(11):1143-1145, 2009.

[13] M. Ueda, Y. Kaneko, Y. Nishitani, T. Morie, and E. Fujii. Biologically-inspired learning device using three-terminal ferroelectric memristor. In Device Research Conference (DRC), 2012 70th Annual, pages 275-276, 2012.

[14] W. Arden, M. Brillouet, P. Cogez, M. Graef, B. Huizing, and R. Mahnkopf. More than moore.

[15] Kunle Olukotun, Basem A Nayfeh, Lance Hammond, Ken Wilson, and Kunyung Chang. The case for a single-chip multiprocessor. ACM Sigplan Notices, 31(9):2-11, 1996.

[16] Behtash Behin-Aein, Deepanjan Datta, Sayeef Salahuddin, and Supriyo Datta. Proposal for an all-spin logic device with built-in memory. Nat Nano, 5(4):266-270, 42010. 
[17] Noel D'Souza, Jayasimha Atulasimha, and Supriyo Bandyopadhyay. Four-state nanomagnetic logic using multiferroics. Journal of Physics D: Applied Physics, 44(26):265001, 2011.

[18] K. Munira, W.A. Soffa, and A.W. Ghosh. Comparative material issues for fast reliable switching in stt-rams. In Nanotechnology (IEEE-NANO), 2011 11th IEEE Conference on, pages 1403 -1408 , aug. 2011.

[19] Kelin J Kuhn, Anand Murthy, Roza Kotlyar, and Markus Kuhn. Past, present and future: Sige and cmos transistor scaling. ECS Transactions, 33(6):3-17, 2010.

[20] Adrian Bachtold, Peter Hadley, Takeshi Nakanishi, and Cees Dekker. Logic circuits with carbon nanotube transistors. Science, 294(5545):1317-1320, 2001.

[21] R. Martel, H.-S.P. Wong, K. Chan, and P. Avouris. Carbon nanotube field effect transistors for logic applications. In Electron Devices Meeting, 2001. IEDM Technical Digest. International, pages 7.5.1 -7.5.4, 2001 .

[22] P. Avouris, J. Appenzeller, R. Martel, and S.J. Wind. Carbon nanotube electronics. Proceedings of the IEEE, 91(11):1772 - 1784, nov 2003.

[23] Jie Deng, N. Patil, Koungmin Ryu, A. Badmaev, Chongwu Zhou, S. Mitra, and H.-S.P. Wong. Carbon nanotube transistor circuits: Circuit-level performance benchmarking and design options for living with imperfections. In Solid-State Circuits Conference, 200\%. ISSCC 2007. Digest of Technical Papers. IEEE International, pages 70 -588, feb. 2007.

[24] D. Unluer, F. Tseng, and A.W. Ghosh. Physics-based gnrfet compact model for digital circuit design. In Semiconductor Device Research Symposium (ISDRS), 2011 International, pages 1 -2 , dec. 2011.

[25] Taisuke Ohta, Aaron Bostwick, Thomas Seyller, Karsten Horn, and Eli Rotenberg. Controlling the electronic structure of bilayer graphene. Science, 313(5789):951-954, 2006.

[26] S. Y. Zhou, G.-H. Gweon, A. V. Fedorov, P. N. First, W. A. de Heer, D.-H. Lee, F. Guinea, A. H. Castro Neto, and A. Lanzara. Substrate-induced bandgap opening in epitaxial graphene. Nature Materials, 6:770, 2007.

[27] Xiaolin Li, Xinran Wang, Li Zhang, Sangwon Lee, and Hongjie Dai. Chemically derived, ultrasmooth graphene nanoribbon semiconductors. Science, 319(5867):1229-1232, 2008.

[28] D. Unluer, F. Tseng, A.W. Ghosh, and M.R. Stan. Monolithically patterned wide-narrow-wide all-graphene devices. Nanotechnology, IEEE Transactions on, 10(5):931 -939, sept. 2011.

[29] F. Tseng, D. Unluer, M. Stan, and A. W. Ghosh. Graphene Nanoelectronics. Springer, 2011.

[30] Young-Woo Son, Marvin L. Cohen, and Steven G. Louie. Energy gaps in graphene nanoribbons. Phys. Rev. Lett., 97(21):216803, Nov 2006.

[31] V.V. Zhirnov, III Cavin, R.K., J.A. Hutchby, and G.I. Bourianoff. Limits to binary logic switch scaling - a gedanken model. Proceedings of the IEEE, 91(11):1934-1939, nov 2003.

[32] Marcelo O. Magnasco. Forced thermal ratchets. Phys. Rev. Lett., 71:1477-1481, Sep 1993.

[33] Vinay Saripalli, Vijay Narayanan, and Suman Datta. Ultra low energy binary decision diagram circuits using few electron transistors. In Nano-Net, volume 20 of Lecture Notes of the Institute for Computer Sciences, Social Informatics and Telecommunications Engineering, pages 200209. Springer Berlin Heidelberg, 2009. 10.1007/978-3-642-04850-0-27.

[34] William M. Reddick and Gehan A. J. Amaratunga. Silicon surface tunnel transistor. Applied Physics Letters, 67(4):494-496, 1995. 
[35] Qin Zhang, Wei Zhao, and A. Seabaugh. Low-subthreshold-swing tunnel transistors. Electron Device Letters, IEEE, 27(4):297-300, 2006.

[36] P.-F. Wang, K. Hilsenbeck, Th. Nirschl, M. Oswald, Ch. Stepper, M. Weis, D. SchmittLandsiedel, and W. Hansch. Complementary tunneling transistor for low power application. Solid-State Electronics, 48(12):2281 - 2286, 2004.

[37] Avik W. Ghosh, Titash Rakshit, and Supriyo Datta. Gating of a molecular transistor: electrostatic and conformational. Nano Letters, 4(4):565-568, 2004.

[38] D. Unluer and A. W. Ghosh. Subthermal switching with nanomechanical relays. 2013.

[39] Jaeseok Jeon, V. Pott, Hei Kam, R. Nathanael, E. Alon, and Tsu-Jae King Liu. Perfectly complementary relay design for digital logic applications. Electron Device Letters, IEEE, 31(4):371 -373, april 2010.

[40] Soogine Chong, K. Akarvardar, R. Parsa, Jun-Bo Yoon, R.T. Howe, S. Mitra, and H.-S.P. Wong. Nanoelectromechanical (nem) relays integrated with cmos sram for improved stability and low leakage. In Computer-Aided Design - Digest of Technical Papers, 2009. ICCAD 2009. IEEE/ACM International Conference on, pages 478-484, nov. 2009.

[41] Mark W Barnett and Philip M Larkman. The action potential. Practical Neurology, 7(3):192197, 2007.

[42] M. Kabir, D. Unluer, Lijun Li, A.W. Ghosh, and M.R. Stan. Computing with nonequilibrium ratchets. Nanotechnology, IEEE Transactions on, 12(3):330-339, 2013.

[43] Clare Howarth, Padraig Gleeson, and David Attwell. Updated energy budgets for neural computation in the neocortex and cerebellum. J Cereb Blood Flow Metab, 32(7):1222-1232, july 2012 .

[44] R. Dean Astumian. Thermodynamics and kinetics of a brownian motor. Science, 276(5314):917922, 1997.

[45] Cees Dekker. Solid-state nanopores. Nat Nano, 2(4):209-215, 2007.

[46] Sarah Rice, Abel W. Lin, Daniel Safer, Cynthia L. Hart, Nariman Naber, Bridget O. Carragher, Shane M. Cain, Elena Pechatnikova, Elizabeth M. Wilson-Kubalek, Michael Whittaker, Edward Pate, Roger Cooke, Edwin W. Taylor, Ronald A. Milligan, and Ronald D. Vale. A structural change in the kinesin motor protein that drives motility. Nature, 402(6763):778$784,1999$.

[47] The international technology roadmap for semiconductors report 2010 update overview, 2010.

[48] George Oster and Hongyun Wang. Atp synthase: two motors, two fuels. Structure, 7(4):R67 R72, 1999.

[49] Richard Feynman, Robert Leighton, and Matthew Sands. The Feynman Lectures on Physics, volume 1. Addison-Wesley, Boston, second edition, 1963.

[50] P. Jung, J. G. Kissner, and P. Hanggi. Regular and chaotic transport in asymmetric periodic potentials: Inertia ratchets. Phys. Rev. Lett., 76:3436-3439, Apr 1996.

[51] M. Borromeo and F. Marchesoni. Asymmetric confinement in a noisy bistable device. Europhysics Letters, 68(6):783, 2004.

[52] D. Reguera, G. Schmid, P. S. Burada, J. M. Rubi, P. Reimann, and P. Hanggi. Entropic transport: Kinetics, scaling, and control mechanisms. Phys. Rev. Lett., 96:130603, Apr 2006. 
[53] Andrea Liscio, Emanuele Orgiu, Jeffrey M. Mativetsky, Vincenzo Palermo, and Paolo Samori. Bottom-up fabricated asymmetric electrodes for organic electronics. Advanced Materials, 22(44):5018-5023, 2010.

[54] J.-L.A. Yeh, C.-Y. Hui, and N.C. Tien. Electrostatic model for an asymmetric combdrive. Microelectromechanical Systems, Journal of, 9(1):126-135, mar 2000.

[55] Juliette Rousselet, Laurence Salome, Armand Ajdari, and Jacques Prostt. Directional motion of brownian particles induced by a periodic asymmetric potential. Nature, 370(6489):446-447, 1994.

[56] D. Cole, S. Bending, S. Savel'ev, A. Grigorenko, and F. Tamegai, T.and Nori. Ratchet without spatial asymmetry for controlling the motion of magnetic flux quanta using time-asymmetric drives. Nature Materials, 5(4):305-311, 2006.

[57] R. Pierret. Semiconductor Device Fundamentals. Pearson Education, 1996.

[58] S. Datta. Electronic Transport in Mesoscopic Systems. Cambridge Studies in Semiconductor Physics and Microelectronic Engineering. Cambridge University Press, 1997.

[59] R. Knorren and K. H. Bennemann. Dynamics of excited electrons in copper and ferromagnetic transition metals: Theory and experiment. Phys. Rev. B., 61:9427-9440, Apr 2000.

[60] K.S. Yeo and K. Roy. Low voltage, low power VLSI subsystems. McGraw-Hill professional engineering. McGraw-Hill, 2005.

[61] J.S. Denker. A review of adiabatic computing. In Low Power Electronics, 1994. Digest of Technical Papers., IEEE Symposium, pages 94-97, oct 1994.

[62] N. Anuar, Y. Takahashi, and T. Sekine. Two phase clocked adiabatic static cmos logic. In System-on-Chip, 2009. SOC 2009. International Symposium on, pages 083-086, oct. 2009.

[63] J. Blanchard. The History of Electrical Resonance. Bell telephone laboratories Incorporated, 1941.

[64] Youxing Jiang, Alice Lee, Jiayun Chen, Vanessa Ruta, Martine Cadene, Brian T. Chait, and Roderick MacKinnon. X-ray structure of a voltage-dependent k+ channel. Nature, 423(6935):33-41, May 2003.

[65] Declan A. Doyle, Joo Morais Cabral, Richard A. Pfuetzner, Anling Kuo, Jacqueline M. Gulbis, Steven L. Cohen, Brian T. Chait, and Roderick MacKinnon. The structure of the potassium channel: Molecular basis of k+ conduction and selectivity. Science, 280(5360):69-77, 1998.

[66] V. Pott, Hei Kam, R. Nathanael, Jaeseok Jeon, E. Alon, and Tsu-Jae King Liu. Mechanical computing redux: Relays for integrated circuit applications. Proceedings of the IEEE, 98(12):2076 -2094, dec. 2010.

[67] Hei Kam, V. Pott, R. Nathanael, Jaeseok Jeon, E. Alon, and Tsu-Jae King Liu. Design and reliability of a micro-relay technology for zero-standby-power digital logic applications. In Electron Devices Meeting (IEDM), 2009 IEEE International, pages 1 -4, dec. 2009.

[68] Eung Seok Park, Yenhao Chen, Tsu-Jae King Liu, and V. Subramanian. Inkjet-printed microelectro-mechanical switches. In Electron Devices Meeting (IEDM), 2011 IEEE International, pages 29.2.1 -29.2.4, dec. 2011.

[69] D. Grogg, Yu Pu, A. Knoll, U. Duerig, U. Drechsler, C. Hagleitner, and M. Despont. Nem switch technologies for low-power logic applications. In Sensors, 2012 IEEE, pages 1-3, 2012. 
[70] K. Akarvardar, D. Elata, R. Parsa, G.C. Wan, K. Yoo, J. Provine, P. Peumans, R.T. Howe, and H.-S.P. Wong. Design considerations for complementary nanoelectromechanical logic gates. In Electron Devices Meeting, 200\%. IEDM 200\%. IEEE International, pages 299 -302, dec. 2007.

[71] C. Hibert and G. A. Racine. Modeling and design of a low-voltage soi suspended-gate mosfet (sg-mosfet) with a metal-over-gate architecture. In Proceedings of the 3rd International Symposium on Quality Electronic Design, ISQED '02, pages 496-, Washington, DC, USA, 2002. IEEE Computer Society.

[72] N. Abele, V. Pott, K. Boucart, F. Casset, K. Segueni, P. Ancey, and A.M. Ionescu. Comparison of rsg-mosfet and capacitive mems resonator detection. Electronics Letters, 41(5):242 -244, march 2005 .

[73] Hei Kam, D.T. Lee, R.T. Howe, and Tsu-Jae King. A new nano-electro-mechanical field effect transistor (nemfet) design for low-power electronics. In Electron Devices Meeting, 2005. IEDM Technical Digest. IEEE International, pages 463 -466, dec. 2005.

[74] Stefano Lenci and Giuseppe Rega. Control of pull-in dynamics in a nonlinear thermoelastic electrically actuated microbeam. Journal of Micromechanics and Microengineering, 16(2):390, 2006 .

[75] S. Krylov, S. Seretensky, and D. Schreiber. Pull-in behavior and multistability of a curved microbeam actuated by a distributed electrostatic force. In Micro Electro Mechanical Systems, 2008. MEMS 2008. IEEE 21st International Conference on, pages 499 -502, jan. 2008.

[76] R. Nadal-Guardia, A. Dehe, R. Aigner, and L.M. Castaner. Current drive methods to extend the range of travel of electrostatic microactuators beyond the voltage pull-in point. Microelectromechanical Systems, Journal of, 11(3):255 -263, jun 2002.

[77] Albert C. J. Luo and Fei-Yue Wang. Nonlinear dynamics of a micro-electro-mechanical system with time-varying capacitors. Journal of Vibration and Acoustics, 126(1):77-83, 2004.

[78] Narayan Aluru. Hierarchical physical models for analysis of electrostatic nanoelectromechanical systems (nems), 2006.

[79] G. Li and N.R. Aluru. Linear, nonlinear and mixed-regime analysis of electrostatic mems. Sensors and Actuators A: Physical, 91(3):278-291, 2001.

[80] G.M. Rebeiz. RF MEMS: Theory, Design, and Technology. Wiley, 2004.

[81] S. Datta. Electronic Transport in Mesoscopic Systems. Cambridge Studies in Semiconductor Physics and Microelectronic Engineering. Cambridge University Press, 1997.

[82] J.A. Knapp and M.P. de Boer. Mechanics of microcantilever beams subject to combined electrostatic and adhesive forces. Microelectromechanical Systems, Journal of, 11(6):754 - 764, $\operatorname{dec} 2002$.

[83] D. Lee, T. Osabe, and Tsu-Jae King Liu. Scaling limitations for flexural beams used in electromechanical devices. Electron Devices, IEEE Transactions on, 56(4):688 -691, april 2009.

[84] Eugene M. Izhikevich. Dynamical Systems in Neuroscience: The Geometry of Excitability and Bursting. MIT Press, 2007.

[85] Choe Senyon. Potassium channel structure. Nat Rev Neurosci, 3:115-121, 2002.

[86] Patrick Crotty and William B Levy. Intersymbol interference in axonal transmission. Neurocomputing, 69(1012):1006 - 1009, 2006. ¡ce:title_Computational Neuroscience: Trends in Research 2006i/ce:title i jxocs:full-name ¿Computational Neuroscience 2005i/xocs:full-name $;.$ 
[87] Patrick Crotty and William B Levy. Energy-efficient interspike interval codes. Neurocomput., 65-66:371-378, June 2005.

[88] Patrick Crotty, Thomas Sangrey, and William B Levy. Metabolic energy cost of action potential velocity. Journal of Neurophysiology, 96(3):1237-1246, September 2006.

[89] Patrick Crotty and William B. Levy. Effects of na+ channel inactivation kinetics on metabolic energy costs of action potentials. Neurocomputing, 70(1012):1652 - 1656, 2007. ¡ce:title ¿Computational Neuroscience: Trends in Research 2007i/ce:title ¿ ixocs:fullname ¿Computational Neuroscience 2006i/xocs:full-namę.

[90] Alfred Renyi. On measures of entorpy and information. In Proc. Fourth Berkeley Sump. on Math. Statist. and Prob., volume 1, pages 547-561. Univ. of Calif. Press, 1961.

[91] K. Moradmand and M. D. Goldfinger. Computation of long-distance propagation of impulses elicited by poisson-process stimulation. Journal of Neurophysiology, 74(6):2415-26, dec 1995.

[92] A.L. Hodgkin and A.F. Huxley. A quantitative description of membrane current and its application to conduction and excitation in nerve. The Journal of Physiology, 1952.

[93] C. A. Vandenberg and F. Bezanilla. Single-channel, macroscopic, and gating currents from sodium channels in the squid giant axon. Biophysical journal, 60(6):1499-1510, December 1991.

[94] Supriyo Datta. Quantum Transport: Atom to Transistor. Cambridge University Press, 2005. 